\title{
A taxonomic revision of the myrmecophilous species of the rattan genus Korthalsia (Arecaceae)
}

\author{
Salwa Shahimi ${ }^{1,2,3}$, Maria Conejero ${ }^{2}$, Christina J. Prychid ${ }^{2}$, Paula J. Rudall ${ }^{2}$, Julie A. Hawkins ${ }^{1}$ \& \\ William J. Baker ${ }^{2}$ (D)
}

Summary. The rattan genus Korthalsia Blume (Arecaceae: Calamoideae: Calameae) is widespread in the Malesian region. Among the 28 accepted species are 10 species that form intimate associations with ants. The ants inhabit the conspicuous ocreas that are produced by these species, using them as domatia to care for their young and aphids. As a foundation for future work, we present here a taxonomic treatment of the myrmecophilous Korthalsia species, based on extensive research pursued both in the herbarium and the field. In addition, we conduct detailed morphological characterisation of the structure and development of ocrea using light and scanning electron microscopy. Descriptions, illustrations, keys and distribution maps are presented for all 10 species, along with microscopic images of ocrea morphology and development for selected species.

Key Words. Ant-plant mutualism, Calamoideae, domatia, Malesia, morphology, myrmecophily, ocrea, taxonomy.

\section{Introduction}

Korthalsia Blume (1843) is one of the eight genera of rattans, which are spiny climbing palms in the subfamily Calamoideae (Baker et al. 2000; Couvreur et al. 2015; Vorontsova et al. 2016). The genus is distributed from northern Indochina, Burma and the Andaman Islands south-eastwards to Sulawesi and New Guinea (Dransfield et al. 2008). Currently, 28 species are accepted (WCSP 2017). The highest diversity of Korthalsia is found in Borneo, Malay Peninsula and Sumatra, with 15, nine and nine species respectively. Korthalsia is an isolated group within the Calamoideae, being the sole genus of subtribe Korthalsiinae in tribe Calameae of subfamily Calamoideae (Dransfield et al. 2008).

Korthalsia is highly distinctive among rattans in its morphology. It has a unique combination of characters, including (usually) diamond-shaped leaflets with jagged distal margins, aerially branching stems, hapaxanthic stems (that die after a single flowering event) and inflorescences with catkin-like rachillae. In addition, all species bear a conspicuous extension of the leaf sheath above the point of attachment of the leaf petiole. This structure, known as an ocrea, is diverse in form in Korthalsia. Dransfield (1981) classified the ocreas of Korthalsia into four types: inflated, divergent, tightly sheathing and fibrous net-like.

The ten species of Korthalsia that possess either inflated or divergent ocreas form mutualistic relationships with ants, which exploit the ocreas as domatia (Dransfield et al. 2008). The ants nest within the ocreas, brooding their young and aphids from which they harvest honeydew. The ants defend the rattan by attacking any animal that disturbs it. In addition, ants that occupy the two species with divergent ocreas ( $K$. hispida and $K$. robusta) make a pulsing, hissing sound when the rattan is disturbed by rhythmically striking their mandibles against the dry wall of the ocrea. Several ant genera have been recorded inhabiting Korthalsia ocreas: Camponotus, Crematogaster, Dolichoderus, Iridomyrmex or Philidris (Bequaert \& Wheeler 1922; Dransfield 1981; Mattes et al. 1998; Moog et al. 2003; Edwards et al. 2010; Chan et al. 2012). This association does not occur throughout the distribution of Korthalsia, the myrmecophilous species being restricted to Borneo, Malay Peninsula, Philippines (Palawan and Mindanao), Singapore and Thailand (Map 1). Although phylogenetic evidence is limited for Korthalsia, recent data indicate that the species with inflated and divergent ocreas are closely related to each other, potentially forming a monophyletic group (Shahimi 2018). Curiously, inflated ocreas occupied by ants occur in other rattan genera, specifically the African genus Laccosperma (Sunderland 2004, 2012) and some species of Calamus, especially on the island of $\mathrm{New}$ Guinea (e.g. Baker \& Dransfield 2002; Dransfield \& Baker 2003; Merklinger et al. 2014)

\footnotetext{
Accepted for publication 25 October 2019. Published online 21 December 2019

1 School of Biological Sciences, Harborne Building, University of Reading, Whiteknights, Reading, RG6 6AS, UK.

2 Royal Botanic Gardens, Kew, Richmond, Surrey, TW9 3AE, UK.

3 Fakulti Sains dan Sekitaran Marin, Universiti Malaysia Terengganu, 21030, Kuala Nerus, Terengganu, Malaysia. e-mail: salwa.shahimi@umt.edu.my
} 


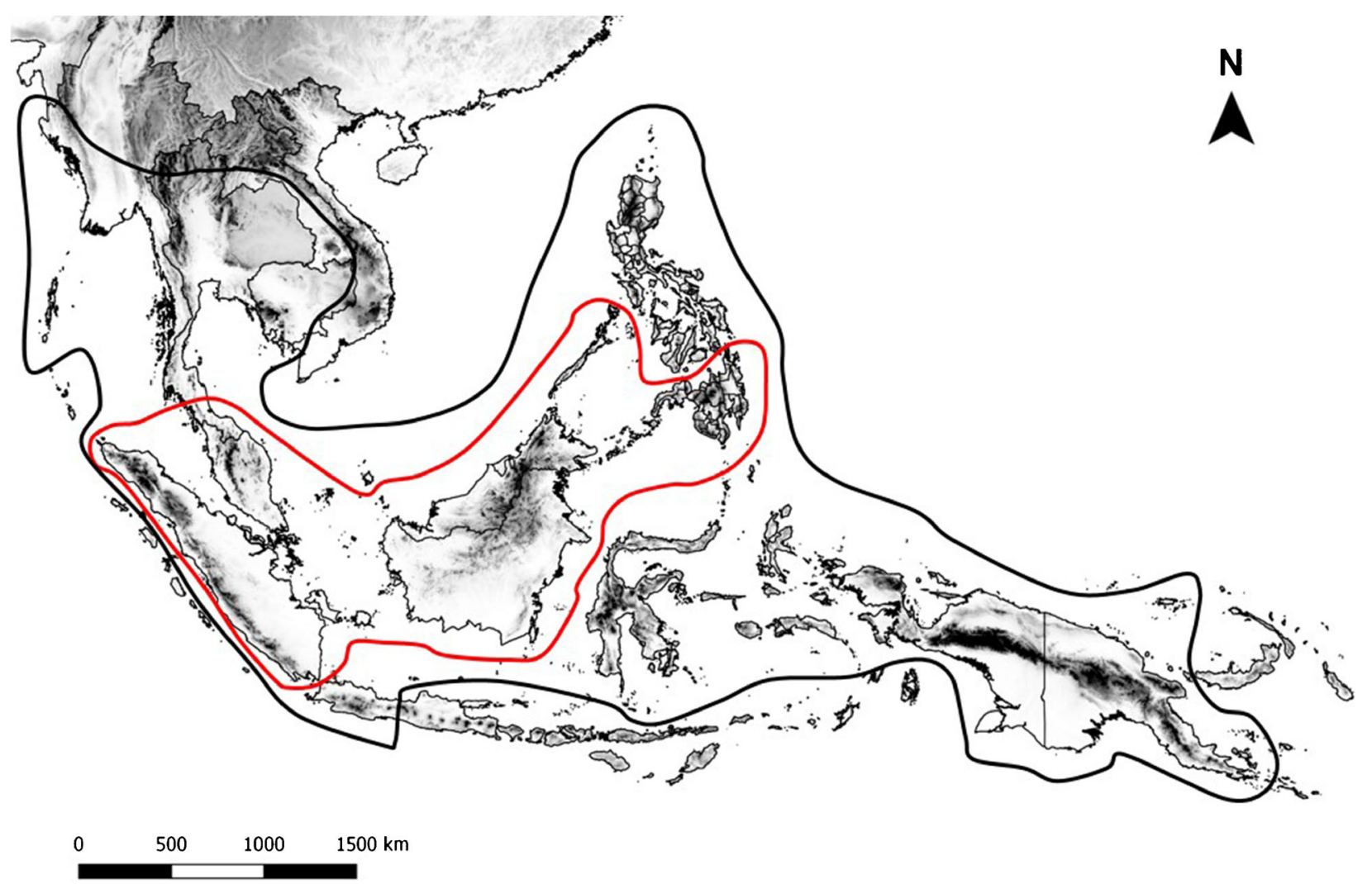

Map 1. Distribution map of the genus Korthalsia in the Malesian region (black line). The distribution of the myrmecophilous species is shown with a red line.

The genus Korthalsia has been known to scientists for nearly two centuries, having been first described in 1843 by Carl Ludwig Blume. However, the last treatment of the genus in its entirety was published 100 years ago (Beccari 1918), building on a series of earlier contributions (Beccari 1884, 1893,1909). Since that time, a synoptic treatment has been published (Dransfield 1981), as well as a series of regional accounts (Furtado 1951; Dransfield 1979, 1984, 1992, 1997; Henderson 2009; Lapis 2010) and some new species (Dransfield 1981; Henderson \& Nguyen 2013), but no complete, modern taxonomic revision has yet been produced. Here we address this knowledge gap by presenting a taxonomic account of the myrmecophilous species of Korthalsia across their entire geographical range. We focus especially on the structural biology of the ocrea, characterising its morphology and development with light and scanning electron microscopy. This revision will serve as a valuable tool and data source for those with special interests in the ecology and evolution of ant-plant mutualism in Korthalsia (e.g. Mattes et al. 1998; Edwards et al. 2010; Chan et al. 2012). Moreover, it represents a contribution towards the completion of a monograph of all species currently recognised in the genus.

\section{Material and Methods}

\section{Taxonomy}

An extensive study of specimens at the Kew herbarium (K) and three international herbaria, namely E, KEP and SING (herbarium acronyms follow Holmgren et al. 1990) underpins this study. Specimen images available online in other institutions were also consulted. Exclamation marks in the taxonomic treatment (!) indicate specimens that have been studied by the authors (including online specimen images). In addition, the first author has conducted two field expeditions in 2014 and 2015, visiting Peninsular Malaysia and Singapore, then Borneo and Singapore. Herbarium specimens were made in the field using standard preparation guidelines for palms (Dransfield 1986; Baker \& Dransfield 2006).

\section{Characterisation of ocrea morphology and development}

Korthalsia stem apices measuring c. $1 \mathrm{~m}$ in length were collected in the field and fixed in $70 \%$ ethanol. Each shoot was carefully dissected by removing leaf sheaths until the shoot was approximately the diameter of a pencil. Images of later stages of development were captured with a photomacroscope. For examination of 
early leaf development close to the stem apex, we used Scanning Electron Microscopy (SEM). Dissected samples were dehydrated through an alcohol series to $100 \%$ ethanol. The samples were transferred to an Autosamdri 815B CPD for critical-point drying, mounted onto SEM pin stubs using double-sided tape, and coated with platinum using an Emitech K550 sputter coater. Samples were examined using a Hitachi S-4700 cold-field emission SEM at RBG Kew. Ocrea morphology was also studied using a Leica photomicroscope M400 (Light Microscopy).

\section{Results}

\section{Habit}

The myrmecophilous species of Korthalsia are moderate to robust, clustering, high-climbing rattans up to $60 \mathrm{~m}$ or more. Some species can reach the forest canopy. They are found only in lowland and hill tropical forest, being absent in montane forest. Most of the species have a wide ecological range and are abundant in primary forest.

\section{Stem}

The stem size of myrmecophilous Korthalsia varies from slender $(0.2-0.8 \mathrm{~cm}$ in diameter without sheath) to moderately large $(1.0-4.0 \mathrm{~cm}$ in diameter without sheath). The internodes are elongate and variable in length. Nodal scars of Korthalsia species are often uneven. Aerial branching sometimes occurs due to parallel forking of stems.

\section{Leaves}

The leaf sheath is tubular, green and usually with caducous indumentum. The sheath is sometimes unarmed or variously armed with spines. The petiole ranges from $1.5 \mathrm{~cm}$ to $40 \mathrm{~cm}$. The leaflets are regularly arranged and usually rhomboid with distal margins praemorse, but in few species (e.g. Korthalsia echinometra), they are lanceolate, although still with a praemorse distal margin. The adaxial surface of the leaflets is usually bright or dark green, and the abaxial surface is typically covered in white or grey indumentum, or sometimes with caducous, orange or brown to dark brown indumentum. The number of leaflets on each side of the rachis varies from one to 25 , with the smallest number belonging to $K$. furcata and the highest in $K$. echinometra. The main veins diverge from the leaflet base. All species of Korthalsia have leaves armed with a cirrus, a leaf whip extending beyond the terminal leaflets.

\section{Ocrea}

We examined ocrea development in four species of myrmecophilous Korthalsia for which fixed material was available, representing both inflated and divergent ocrea types (sensu Dransfield 1981): inflated ocrea (K. echinometra, K. scortechinii, and $K$. rostrata), divergent ocrea ( $K$. robusta). For comparison, we also examined three species with the tightly sheathing ocrea type ( $K$. debilis, $K$. rigida and $K$. tenuissima). The fibrous net-like ocrea type is found only in $K$. jala, which was not encountered during our fieldwork and fixed material was not available (Fig. 1).

The three species examined with inflated ocreas (Fig. 2A - C) differ in the origin of the inflation. Korthalsia echinometra (Fig. 2A) shows inflation from the point of attachment of the ocrea to the leaf sheath, whereas in the other two species (Fig. 2B, C) the ocrea is tightly clasping at the base and inflated above. At the stage recorded in Fig. 2, the tightly clasping part is almost as long as the inflated part, although in the mature ocrea the clasping part is relatively shorter. Korthalsia echinometra also differs from $K$. scortechinii and $K$. rostrata in that it lacks the notch that forms in the ocrea apex of $K$. scortechinii and $K$. rostrata. In contrast, the apex is truncate in the species in which the ocrea is tightly sheathing for its entire length $(K$. debilis and $K$. rigida; Fig. $2 \mathrm{E}-\mathrm{F}$ ). In some species, spines cover the ocrea at an early stage (Fig. 2A - D), whereas in others they are absent (Fig. 2E - F).

At an earlier stage, the degree of ocrea inflation differs between Korthalsia echinometra, K. scortechinii and $K$. rostrata (Fig. 3A-C). At this stage, there is slight inflation of the leaf base in K. echinometra (Fig. 3A), no inflation in K. scortechinii (Fig. 3B) and clear inflation in $K$. rostrata (Fig. 3C). The bifid ocrea apex is clearly visible at this stage in $K$. rostrata (Fig. 3C). Small spines are also visible at this stage on the ocrea of $K$. robusta (Fig. 3D) and a few small rounded spines were visible in $K$. debilis (Fig. 3E), although none was present in K. tenuissima (Fig. 3F).

Young leaf primordia at successive stages were examined for Korthalsia echinometra (Fig. 4) and $K$. debilis (Fig. 5), representing the inflated and tightly sheathing ocrea type respectively. In both species, early stages of leaf plication are evident (Fig. 4B, 5A), although the ocrea lacks plications in either species at any stage. In both species, the ocrea becomes visible at a relatively late stage (Fig. 4D, 5D).

\section{Inflorescence}

The flowering behaviour in all species of Korthalsia is hapaxanthic (individual stems flower only once in their lifetime and die subsequently). The inflorescences are borne at the apex of the stem and are lax to congested, with one to two orders of branching. The peduncle is adnate to the internode above the subtending leaf (Dransfield et al. 2008). The prophyll is 2-keeled and tightly sheathing. Rachis bracts are similar to the prophyll, but lack keels and are somewhat inflated in some species. 

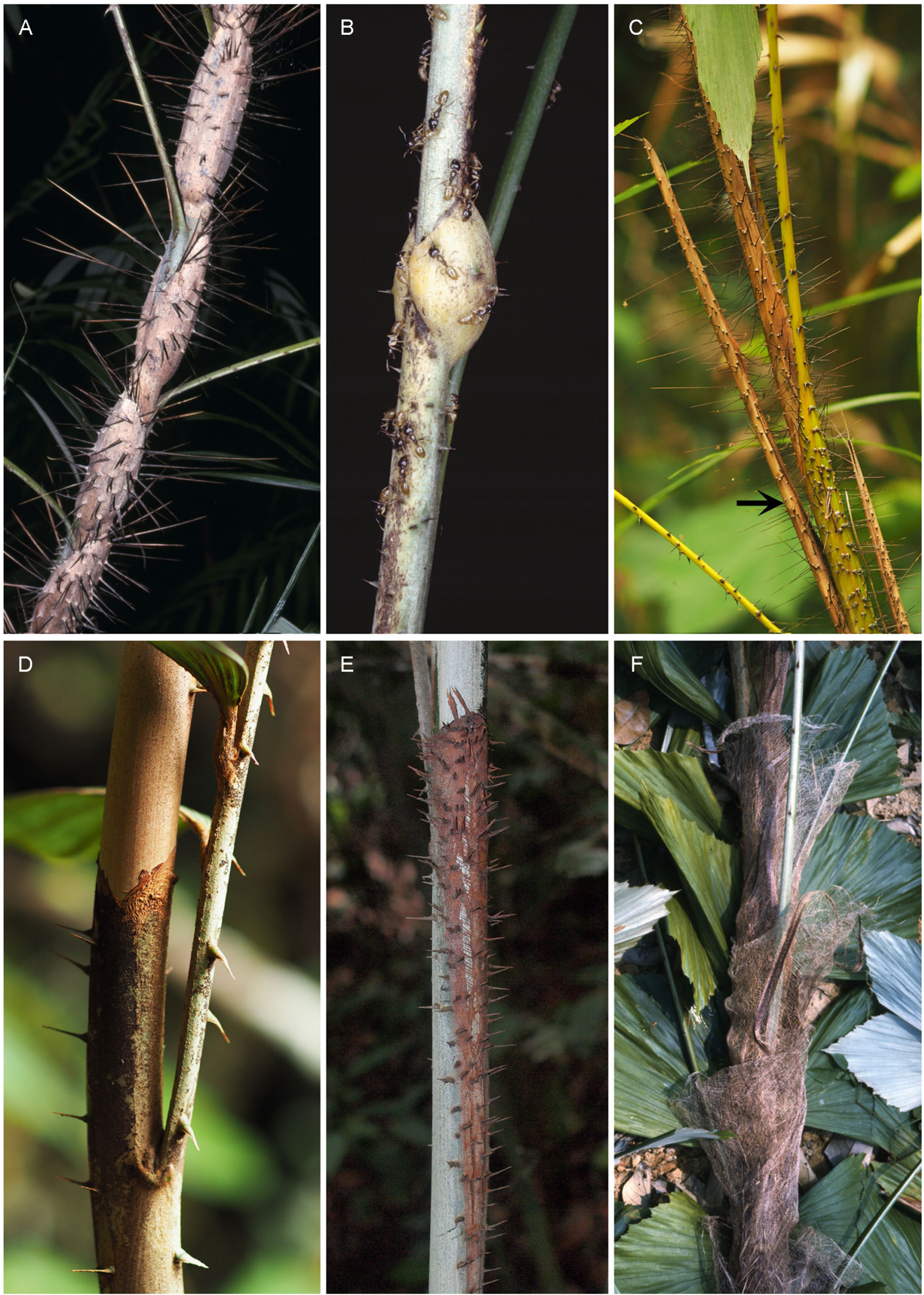

Fig. 1. Images taken in the field of the leaf bases of six species of Korthalsia with different ocrea types. A $K$. echinometra, inflated ocrea; B K. rostrata, inflated ocrea; C K. robusta, divergent ocrea; D K. debilis, tightly sheathing ocrea; E K. rigida, tightly sheathing ocrea; F K. jala, fibrous net-like ocrea. PHOTOS: A, B WILLIAM BAKER, C - E SALWA SHAHIMI, F JOHN DRANSFIELD. 

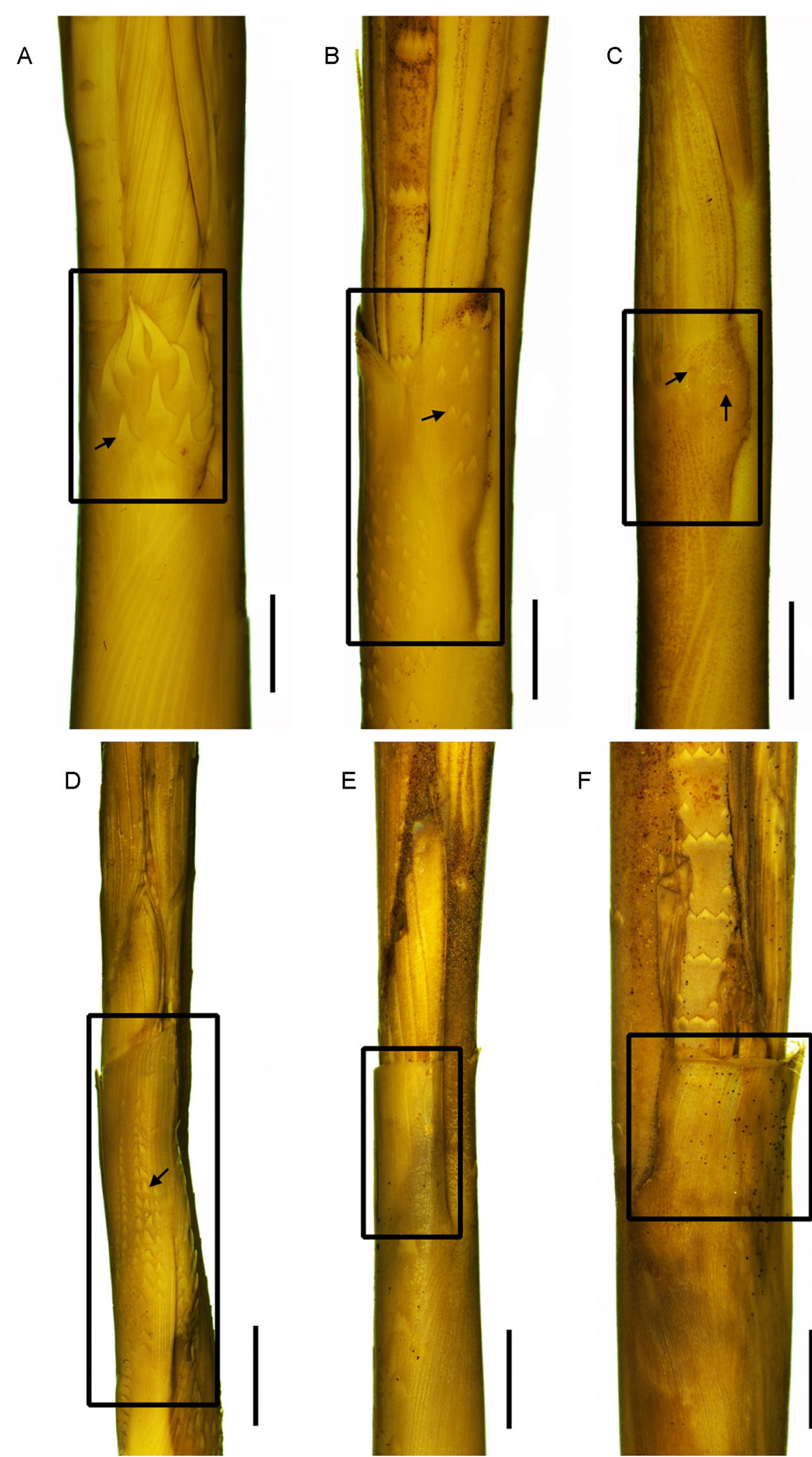

E

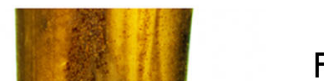

F

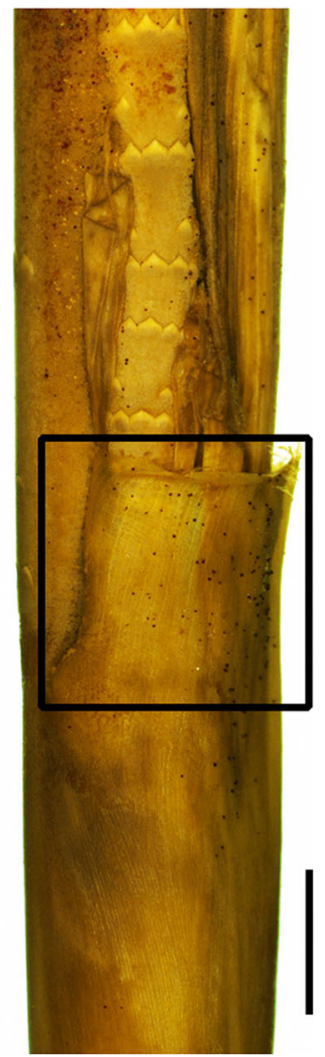

Fig. 2. Photomacroscope images of leaf bases in fixed material. $A-C$ represent species with an inflated ocrea, $D$ a divergent ocrea, E - F a tightly sheathing ocrea. A Korthalsia echinometra; B K. scortechinii; C K. rostrata; D K. robusta; E K. debilis; F K. rigida. Boxed areas outline the region of the developing ocrea. Arrows indicate spines. Bar $=10 \mathrm{~mm}$. 

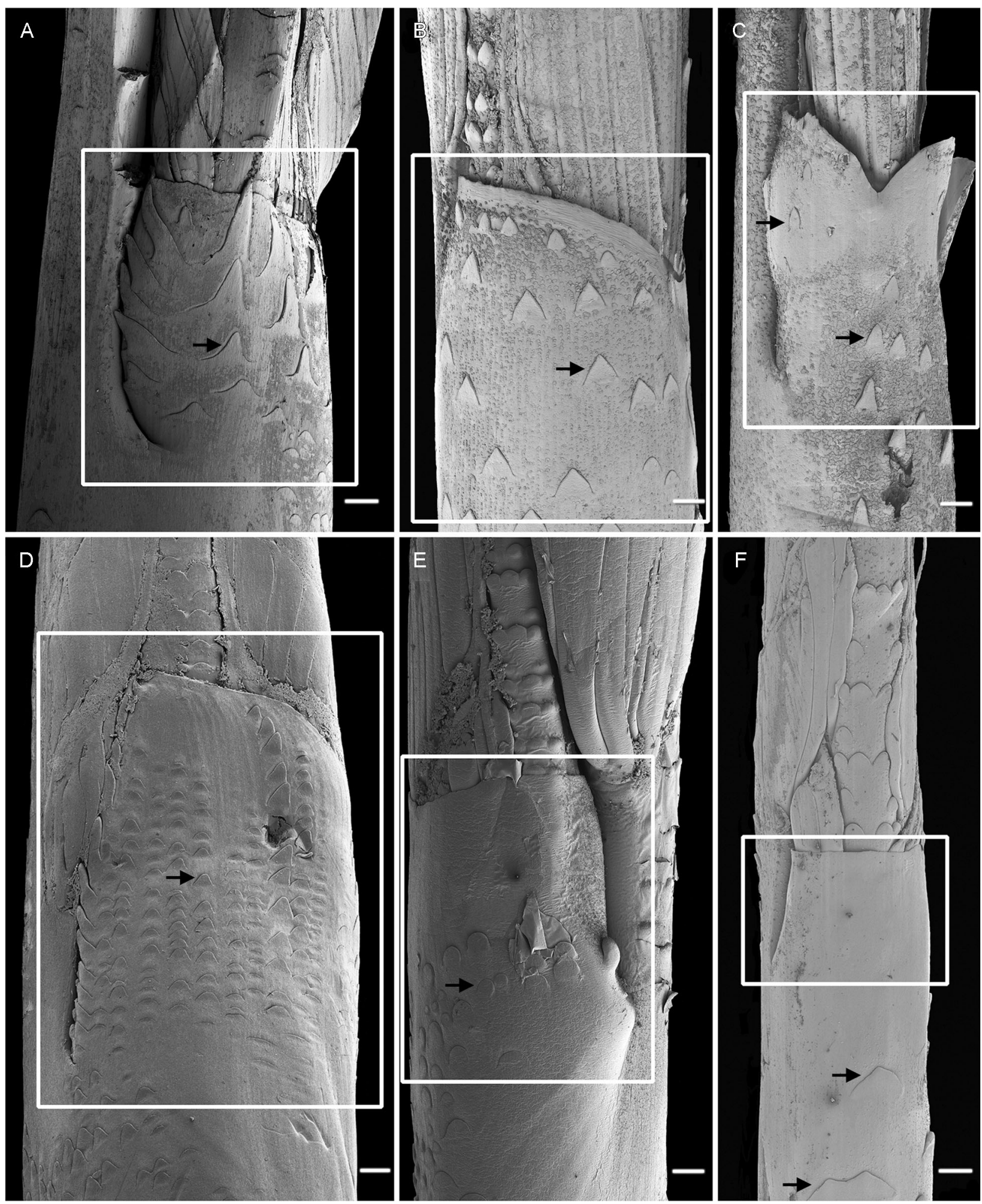

Fig. 3. SEM images of dissected bases of very young leaves, showing early stages of ocrea development. A - C represent species with an inflated ocrea, D a divergent ocrea, E - F a tightly sheathing ocrea. A Korthalsia echinometra; B K. scortechinii; C K. rostrata; D K. robusta; E K. debilis; F K. tenuissima. Boxed areas outline the region of the developing ocrea. Arrows indicate spines. Bars $=100 \mu \mathrm{m}$. 

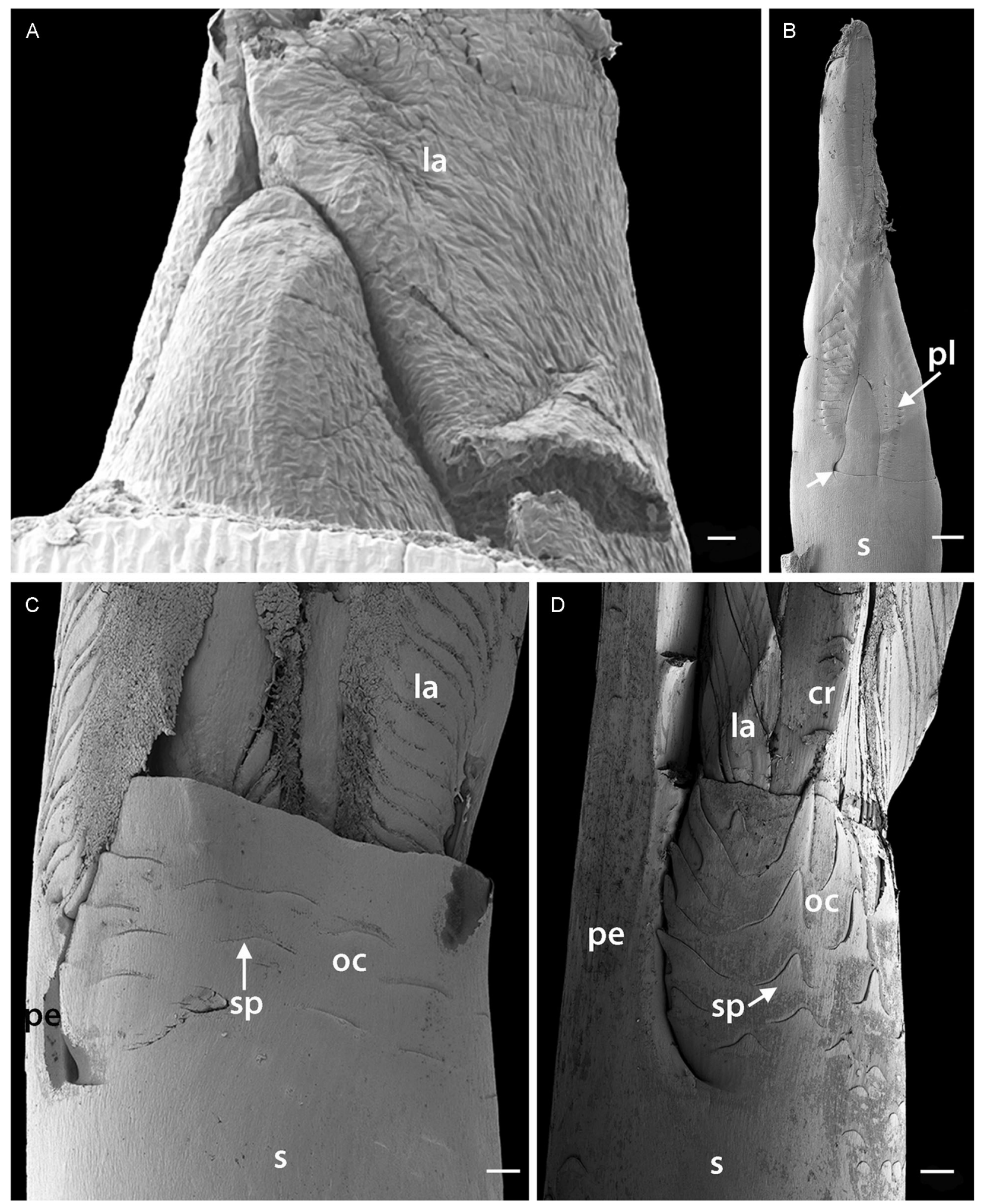

Fig. 4. Leaf developmental series (SEM). A - D developmental stages for Korthalsia echinometra, a species with an inflated ocrea. A leaf primordium differentiated into a distal lamina but lacking plications at this stage; B successive stages of leaf elongation, with leaf plication becoming more pronounced on both sides of the lamina and small lobes present at the top of the leaf sheath (arrow) indicating the first stage of ocrea development; $\mathbf{C}$ at this stage, the petiole has begun to elongate, the spines have begun to develop and the ocrea is more clearly visible; $\mathrm{D}$ the ocrea is a well-delimited structure that will persist into the adult organ. Bars = $100 \mu \mathrm{m}$. la lamina, s sheath, sp spines, pe petiole, pl leaf plications, oc ocrea. 

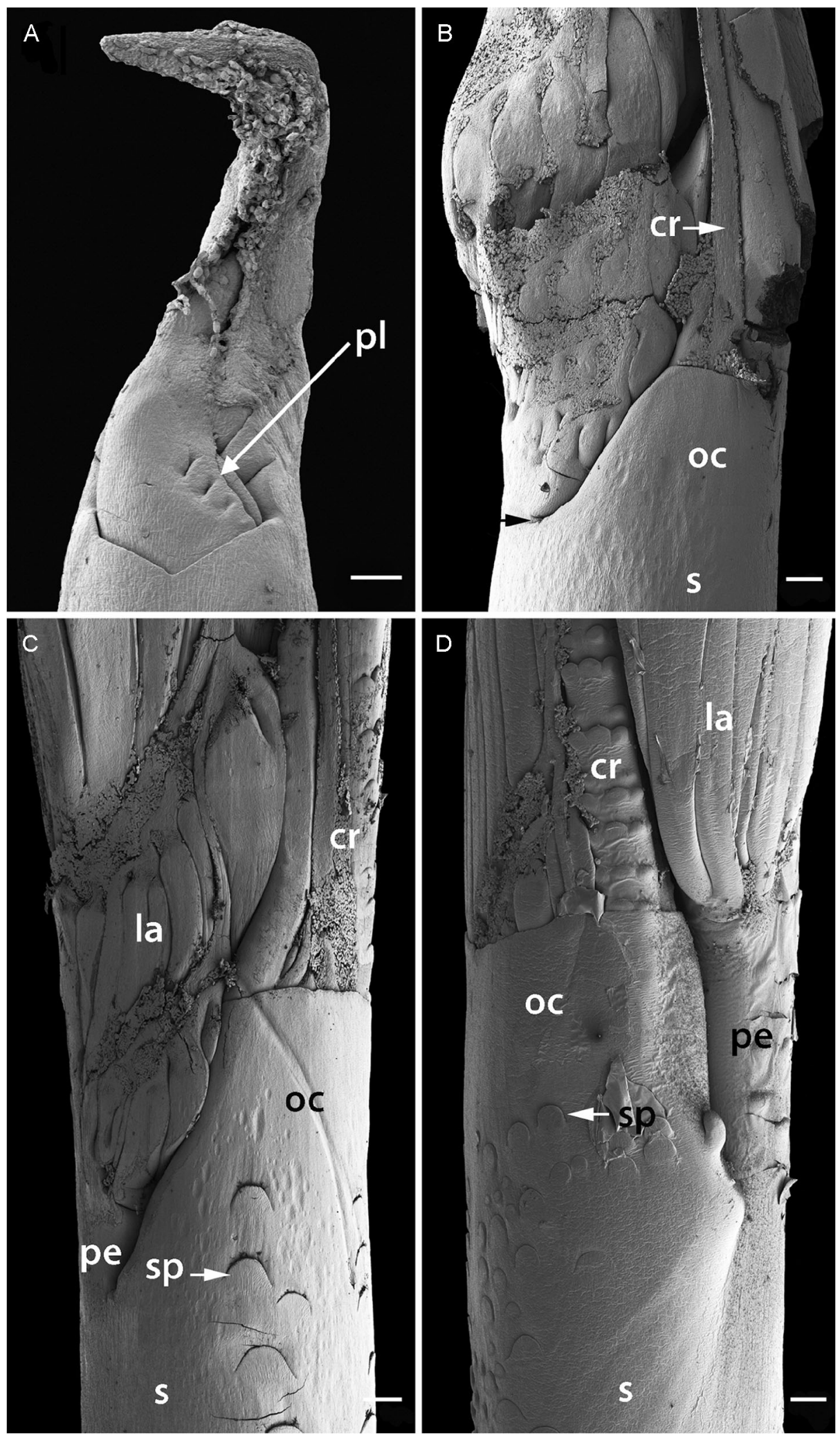

Fig. 5. Leaf developmental series (SEM). A - D developmental stages for Korthalsia debilis, a species with a tightly sheathing ocrea. A plication inception is visible on the leaf; $\mathbf{B}$ the cirrus is visible above the petiole (arrow); $\mathbf{C}$ at this stage, the petiole has begun to elongate, the spines have begun to develop and the ocrea has become more visible; $\mathbf{D}$ the ocrea is a well-delimited structure that will persist into the adult organ. Bars $=100 \mu \mathrm{m}$. $\mathrm{s}$ sheath, sp spines, cr cirrus, pe petiole, pl leaf plications, oc ocrea. 
The bracts can be unarmed or sparsely armed and densely covered with caducous indumentum. The rachillae are cylindrical and catkin-like, with densely arranged rachilla bracts, sometimes with hairs in between. The rachillae can be slender or congested.

\section{Flowers}

In all Korthalsia species, the flower is hermaphroditic and borne in pits in the catkin-like rachillae. Unlike many other calamoid palms, the flower is solitary, rather than borne in pairs. The calyx is tubular at the base, with three sepals and usually shorter than corolla. The corolla consists of three valvate petals. The flower contains $6-9$ stamens.

\section{Fruits and Seeds}

The fruit of Korthalsia species examined here is globose to ovoid, with one seed. The epicarp is thin and covered with vertical rows of imbricate scales. The scales are usually brown to strawcoloured. The mesocarp develops as a thin sweetfleshy layer surrounding the seed, and the endocarp is not differentiated. Unlike most species of tribe Calameae, the seed lacks a sarcotesta. The seed is attached basally. In most of the species, the endosperm is ruminate, but it in a few species (e.g. $K$. hispida) the endosperm is homogeneous. The fruits of most species appear to be attractive to animals (Dransfield 1981).

\section{Taxonomic Treatment}

\section{Key to myrmecophilous species of Korthalsia}

1. Ocrea not clasping, diverging at an angle from the stem, margins tending to inroll (Borneo, Peninsular

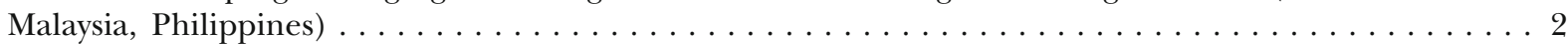
Ocrea clasping the stem and inflated (Borneo, Peninsular Malaysia, Sumatra, Singapore, Thailand, Philippines) . . . 3

2. Ocrea $18.5-30.0 \times 3.0-4.5 \mathrm{~cm}$ long, ocrea, sheath and inflorescences bracts with abundant black spicules

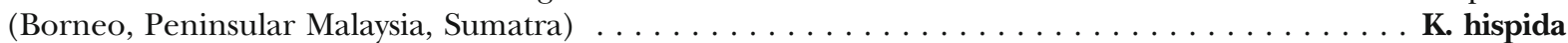
Ocrea $16.0-40.0 \times 3.0-7.0 \mathrm{~cm}$ long, ocrea, sheath and inflorescences bracts without black spicules absent

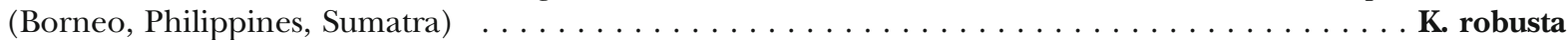

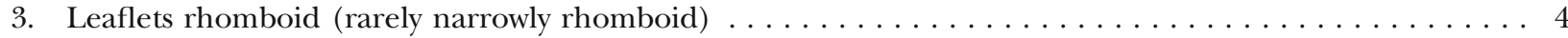
Leaflets narrowly lanceolate (Borneo, Peninsular Malaysia, Singapore, Sumatra) $\ldots \ldots \ldots \ldots \ldots \ldots$ K echinometra

4. Leaflets only one on each side of the rachis (Sarawak) $\ldots \ldots \ldots \ldots \ldots \ldots \ldots \ldots \ldots \ldots \ldots$. furcata Leaflets usually more than three on each side of rachis $\ldots \ldots \ldots \ldots \ldots \ldots \ldots \ldots \ldots \ldots$

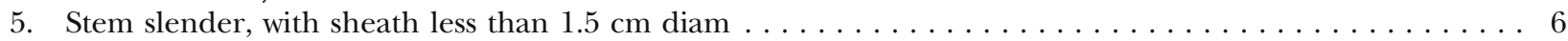
Stem robust, with sheath at least $2.0 \mathrm{~cm}$ diam $\ldots \ldots \ldots \ldots \ldots \ldots \ldots \ldots \ldots \ldots \ldots$

6. Leaflets densely indumentose on undersurface (Philippines) $\ldots \ldots \ldots \ldots \ldots \ldots \ldots$ K. scaphigeroides

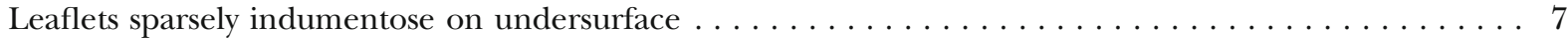

7. Ocrea $2.5-5.0 \times 1.0-3.0 \mathrm{~cm}$, transverse veinlets moderately closely spaced, rachillae slender (Peninsular Malaysia, Borneo, Singapore, Sumatra) $\ldots \ldots \ldots \ldots \ldots \ldots \ldots \ldots \ldots \ldots \ldots \ldots \ldots \ldots \ldots \ldots \ldots$ K. rostrata Ocrea $2.5-8.3 \times 1.0-1.5 \mathrm{~cm}$, transverse veinlets fine and closely spaced, rachillae robust and congested

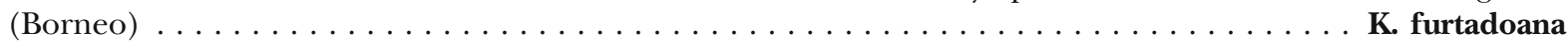

8. Ocrea armed with scattered triangular spines up to $2.0 \mathrm{~cm}$ long and ocrea about $20-26 \mathrm{~cm}$ long . . . 9 Ocrea armed with scattered very short triangular spines about $3 \mathrm{~mm}$ long and ocrea about $10-15 \mathrm{~cm}$ long

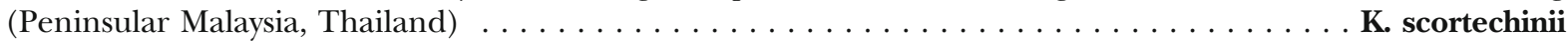

9. Leaflets rhomboid to broadly rhomboid, $6-8$ leaflets each side of rachis and petiole $10-25 \mathrm{~cm}$ long

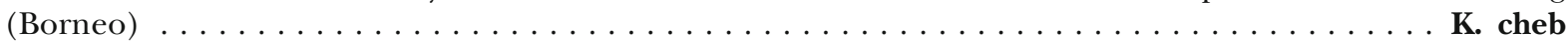
Leaflets rhomboid, 7 - 12 leaflets each side of rachis and very long petiole c. $40 \mathrm{~cm}$ long (Borneo) ... K K angustifolia

1. Korthalsia angustifolia Blume (1843: 172). Type: Indonesia, South Kalimantan, Sungai Dusun, Korthals $\mathcal{E}$ Muller s.n. (holotype L!; isotype FI!).

Clustering rattan climbing to $50 \mathrm{~m}$. Stem with sheaths $35-50 \mathrm{~mm}$ diam., without sheaths $20-30 \mathrm{~mm}$ diam.; internodes $10-25 \mathrm{~cm}$. Leaf $2-3 \mathrm{~m}$ long including cirrus and petiole; sheath green, with grey indumentum, sheath sparsely armed with brown to dark brown triangular spines, spines more abundant near sheath mouth, spines $3-13 \mathrm{~mm}$ long, c. $1 \mathrm{~mm}$ wide; ocrea $24.2-25.5 \times 2.5-3.5 \mathrm{~cm}$, very large and conspicuous, elongate inflated, clasping the stem, tough, brown (when dry), armed with $1.0-2.0 \mathrm{~cm}$ long dark brown, planar spines, ocrea occupied by ants; cirrus c. $1 \mathrm{~m}$; petiole c. $40 \mathrm{~cm}, 10-15 \mathrm{~mm}$ wide and $3-4 \mathrm{~mm}$ thick at base, flattened adaxially, abaxially rounded, with grey indumentum as sheath, 
sparsely armed with spines; rachis c. $0.9 \mathrm{~m}$, armed with recurved grapnel spines organised in whorls; $7-12$ leaflets each side of rachis, regularly arranged, rhomboid, mid-leaf leaflets $18.0-23.0 \times 4.7-5.9 \mathrm{~cm}$, leaflet dark green above, glabrous, with caducous, grey-brown indumentum on undersurface, distal margin conspicuously praemorse, transverse veinlets moderately conspicuous, moderately spaced c. $1.0 \mathrm{~mm}$. Inflorescence lax, $47.0-61.7 \mathrm{~cm}$ long, branched to 2 orders; rachis bracts up to $6.2-7.0 \times 3.5-3.7 \mathrm{~cm}$, tightly sheathing and splitting, with caducous, dark brown indumentum; primary branches $1-2,4.0-8.0 \mathrm{~cm}$ apart, proximal primary branch $20.2-22.1 \mathrm{~cm}$ long, with up to 2 rachillae; rachillae $17.8-24.8 \mathrm{~cm}$ long, including $1.5-3.7 \mathrm{~cm}$ visible stalk, $0.7-1.0 \mathrm{~cm}$ wide, densely hairy between rachilla bracts. Flower not seen. Fruit mature fruit not seen. Seed not seen. (Fig. 6).

DISTRIBUTION. Borneo (Central and South Kaliman$\operatorname{tan)}$ (Map 2).

SPECIMENS EXAMINED. BORNEO. INDONESIA. KALIMANTAN TENGAH: Palangka Jaya, [2 $\left.{ }^{\circ} 12^{\prime} \mathrm{S} 113^{\circ} 50^{\prime} \mathrm{E}\right], 25$ Jan. 1974, Dransfield 3924 (K!, L). KALIMANTAN SELATAN: Barito R., $12 \mathrm{~km}$ upstream of Marabahan, [250'S 114²9'E], 3 Jan. 1989, Geisen 89 (K!); Sampit, Sei Pemalian, [2 ${ }^{\circ} 32^{\prime} \mathrm{S} 112^{\circ} 57^{\prime} \mathrm{E}$ ], July 1986, Sutisna 70 (K!); Sungai Dusun, [1 $\left.{ }^{\circ} 48^{\prime} \mathrm{S} 114^{\circ} 31^{\prime} \mathrm{E}\right]$, Korthals $\mathcal{E}^{\circ}$ Muller s.n. (FI!, L!).

HABITAT. Along river banks and in freshwater swamp on alluvial soils.

VERNACULAR NAMES. Ahas, Rotan ahas, Rotan patung (Indonesia).

USES. Not recorded.

NOTES. Very little herbarium material of Korthalsia angustifolia is available. Although $K$. angustifolia is vegetatively very similar to $K$. cheb and $K$. scortechinii, it has a very long petiole compared to $K$. cheb and K. scortechinii.

2. Korthalsia cheb Becc. (Beccari 1884: 67). Type: Malaysia, Sarawak, Gunung Matang, 1 June 1866, Beccari PB 1936 (holotype FI!).

Robust, clustering rattan climbing to $30 \mathrm{~m}$. Stem with sheaths 20 - $40 \mathrm{~mm}$ diam., without sheaths 10 $25 \mathrm{~mm}$ diam.; internodes $25-30 \mathrm{~cm}$. Leaf $2.2-3.5 \mathrm{~m}$ long including cirrus and petiole; sheath bright green, almost completely obscured by ocrea, with caducous, black indumentum, sheath sparsely armed with brown, triangular spines, spines $7-12 \mathrm{~mm}$ long, c. $2 \mathrm{~mm}$ wide; ocrea $20.0-26.0 \times 5.0-8.0 \mathrm{~cm}$, very large and conspicuous, tightly sheathing above the petiole then inflated, clasping the stem, tough, brown, armed with 6 - $26 \mathrm{~mm}$ long, dark brown, planar spines, ocrea occupied by numerous ants, entry hole made by ants often visible; cirrus $0.7-1.0 \mathrm{~m}$, armed with recurved grapnel spines organised in whorls; petiole $10-25 \mathrm{~cm}$, $14-18 \mathrm{~mm}$ wide and $4-5 \mathrm{~mm}$ thick at base, flattened adaxially, abaxially rounded, with brown indumentum, armed with spines as sheath; rachis $0.80-2.03 \mathrm{~m}$, armed with recurved grapnel spines organised in whorls; $6-8$ leaflets each side of rachis, regularly arranged, rhomboid to broadly rhomboid, mid-leaf leaflets $20.8-27.3 \times 4.3-7.7 \mathrm{~cm}$, leaflet light to dark green above, brownish undersurface covered with caducous, orange brown indumentum when young, distal margin conspicuously praemorse, transverse veinlets conspicuous, moderately closely spaced c. 1.5 $\mathrm{mm}$. Inflorescence lax, 52.9 - $54.0 \mathrm{~cm}$ long including $11.5 \mathrm{~cm}$ peduncle, branched to 2 orders; prophyll 4.5 $-6.5 \times 0.8-1.8 \mathrm{~cm}$; rachis bract $2.0-8.7 \times 1.9-2.3$ $\mathrm{cm}$, tightly sheathing, with caducous, dark brown indumentum; primary branches $1-2,0.5-1.0 \mathrm{~cm}$ apart, proximal primary branch $16.8-21.0 \mathrm{~cm}$ long, with $1-4$ rachillae; rachillae $13.5-23.6 \mathrm{~cm}$ long and slender including $0.5-1.5 \mathrm{~cm}$ visible stalk, $0.5-$ $1.0 \mathrm{~cm}$ wide, densely hairy between rachilla bracts. Flower $4 \times 2 \mathrm{~mm}$ in the bud. Fruit somewhat oblong, 1.4 $-2.2 \times 0.9-1.8 \mathrm{~cm}$ covered in $16-18$ vertical rows of yellow-brown scales. Seed $1.2 \times 0.1 \mathrm{~cm}$; endosperm ruminate. (Fig. 7).

DISTRIBUTION. Borneo (in the 1st Division of Sarawak, Sabah, East and South Kalimantan) (Map 3).

SPECIMENS EXAMINED. BORNEO. INDONESIA. KALIMANTAN TIMUR: Gunung Mendam at foot north of Tabang, $100 \mathrm{~m}$ elev., [0 $0^{\circ} 37^{\prime} \mathrm{N} 115^{\circ} 54$ 'E], 16 Jan. 1979, Mogea et al. 1630 (K!); Surroundings of Long Sungai Barang, $800 \mathrm{~m}$ elev., [1 $\left.{ }^{\circ} 40^{\prime} \mathrm{N} 115^{\circ} 0{ }^{\prime} \mathrm{E}\right], 30$ Dec. 1992, van Valkenburg 1206 (K!). KALIMANTAN SELATAN: Gunung Besar, $800 \mathrm{~m}$ elev., [243'S 115³7'E], 19 Feb. 1979, Mogea et al. 1686 (K!). MALAYSIA. SABAH: Tambunan, Crocker Range National Park NW of Kampung Kuyongon close to Tundulu R., $950 \mathrm{~m}$ elev., [5 40 'N $116^{\circ} 20^{\prime} \mathrm{E}$ ], 13 Sept. 2000, Andersen 175 (C, K!, KEP, SAN); Tambunan, Mile 8 , road to Kampung Kaingaran, 1150 m elev., 26 March 1999, Dewol SAN 141821 (K!, SAN); Kota Kinabalu, Mile 24, Sinsuron Road, Crocker Range, 800 m elev., 21 Aug. 1979, Dransfield et al. 5535 (K!, L, SAF, SAR); Ranau, Kampung Bundu Tuhan, [5 $59^{\circ} \mathrm{N} 116^{\circ} 32^{\prime} \mathrm{E}$ ], 8 Jan. 1994, Soibeh 683 (K!). SARAWAK: Kapit, Batang Balleh, [1 ${ }^{\circ} 50^{\prime} \mathrm{N} 113^{\circ} 40 ' \mathrm{E}$ ], 16 July 1987, Lee $S 54592$ (K!, L); Kapit, Belaga, $200 \mathrm{~m}$ elev., [1 ${ }^{\circ} 55^{\prime} \mathrm{N} 111^{\circ} 11^{\prime} \mathrm{E}$ ], 9 Aug. 1975, Dransfield et al. 4671(K!, KEP); Kuching, 1st Division, Gunung Matang, $750 \mathrm{~m}$ elev., 9 April 1981, Dransfield et al. 5882 (K!, L, NY, PNH); Gunung Matang, [1 ${ }^{\circ} 36^{\prime} \mathrm{N} 110^{\circ} 10^{\prime} \mathrm{E}$ ], 1 June 1866, Beccari PB 1936 (FI!); Kuching, 1st Division, Kampung Kakeng, 200 m elev., [1 ${ }^{\circ} 9^{\prime} \mathrm{N} 110^{\circ} 27^{\prime} \mathrm{E}$ ], 20 July 1993, Bunker 51 (K!); Kuching, Lundu, Waterfall trail Gunung Gading, 

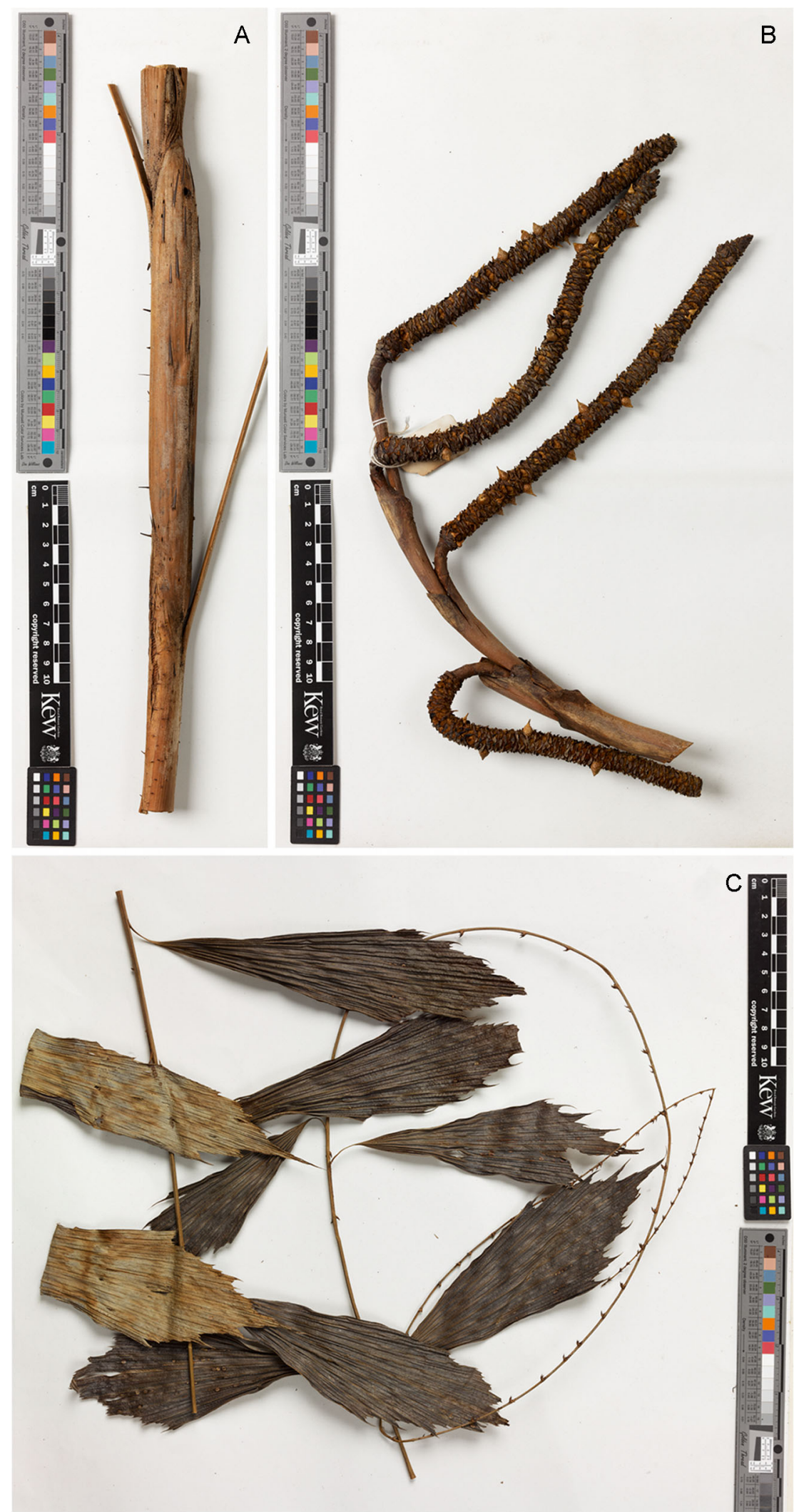

Fig. 6. Korthalsia angustifolia. A ocrea (Sutisna 070); B inflorescence (Giesen 89); C leaflets (Sutisna 070). 


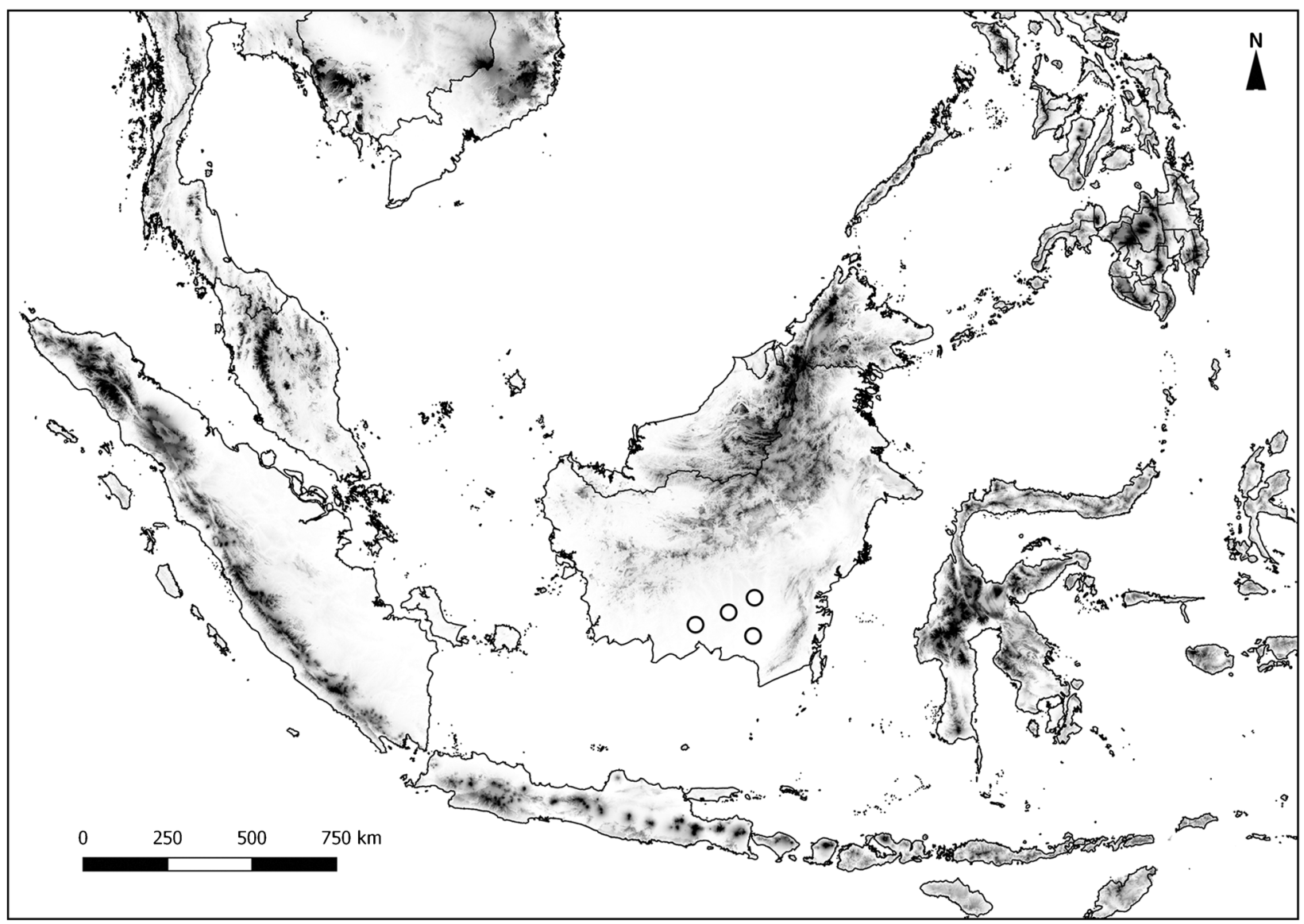

Map 2. Distribution of Korthalsia angustifolia. Dots are point localities of specimens examined.

300 m elev., 25 April 1996, Baker 742 (K!, KEP, SAN, SING!); Kuching, Siburan, Semenggoh Arboretum, [1 $\left.{ }^{\circ} 24^{\prime} \mathrm{N} 110^{\circ} 18^{\prime} \mathrm{E}\right], 9$ Feb. 1995, Baker 513 (K!, KEP).

HABITAT. Hill dipterocarp forest at altitudes up to 800 $\mathrm{m}$, usually in humid valleys.

VERNACULAR NAMES. Wee jematang tengah (Kayan), Lasas (Petung), Wae dura (Penan), Uei sanam (Kenyah), Rua (Bidayuh).

USES. Making baskets, as binding material for constructing pig-sties.

NOTES. Korthalsia cheb is a very distinctive species with its very large inflated ocrea and broad diamondshaped (rhomboid) leaflets. It can be confused with K. angustifolia.

3. Korthalsia echinometra Becc. (Beccari 1884: 66). Type: Malaysia, Sarawak, Gunung Matang, 1 June 1866, Beccari PB 1935 (holotype FI!).

Korthalsia angustifolia var. gracilis Miq. (Miquel 1868:

16). Type: Indonesia, Sumatra, Palembang, De Vriese (holotype L).

Korthalsia horrida Becc. (Beccari 1884: 66). Type: Malaysia, Sarawak: Gunung Matang, 1 June 1866, Beccari PB 1918 (holotype FI!).
Daemonorops ochreata Teijsm. \& Binn. (Teijsmann \& Binnendijk 1866: 74), nom. inval.

Calamus ochreatus Miq. (Miquel 1868: 29), nom. inval.

Moderately robust, clustering rattan climbing to $50 \mathrm{~m}$. Stem with sheaths $12-35 \mathrm{~mm}$ diam., without sheaths 9 $20 \mathrm{~mm}$ diam.; internodes $12-25 \mathrm{~cm}$. Leaf $1.0-2.5 \mathrm{~m}$ long including cirrus and petiole; sheath green, almost entirely obscured by ocrea, with indumentum grey in colour, sheath sparsely armed with scattered, brown triangular spines, spines more abundant near sheath mouth, spines 2-20 mm long, 1 - 3 mm wide; ocrea $6.0-$ $15.5 \times 3.5-5.0 \mathrm{~cm}$, conspicuous, inflated and elongate, clasping the stem, tough, brown, armed with $1.0-7.7 \mathrm{~cm}$ long, dark brown to black, planar spines, ocrea occupied by numerous ants, entry hole made by ants often visible; cirrus $0.75-1.80 \mathrm{~m}$, armed with recurved grapnel spines organised in whorls; petiole $5.0-40.0 \mathrm{~cm}, 7-20 \mathrm{~mm}$ wide and $3-5 \mathrm{~mm}$ thick at base, flattened adaxially, abaxially rounded, with caducous, brown indumentum, sparsely armed with spines; rachis $0.49-1.50 \mathrm{~m}$, armed with recurved grapnel spines at base and then the spines organised in whorls before the cirrus; $8-25$ leaflets each side of rachis, regularly arranged, narrowly lanceolate, mid-leaf leaflets $24.6-31.4 \times 1-2 \mathrm{~cm}$, leaflets dull to dark 


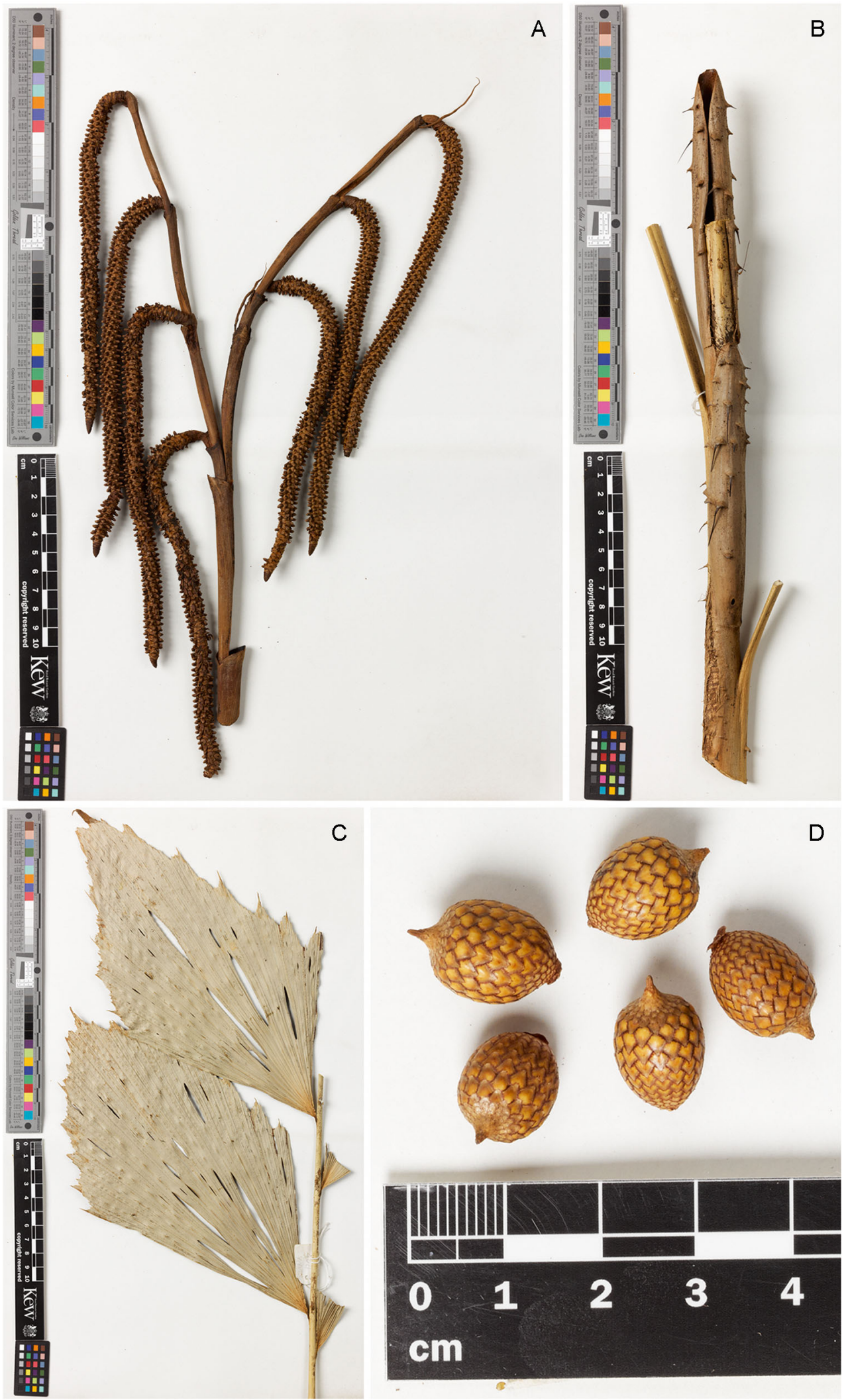

Fig. 7. Korthalsia cheb. A inflorescence (Lee S.54592); B ocrea (Van Valkenburg 1206); C leaflets (Van Valkenburg 1206); D fruit (Dransfield et al. 5882). 


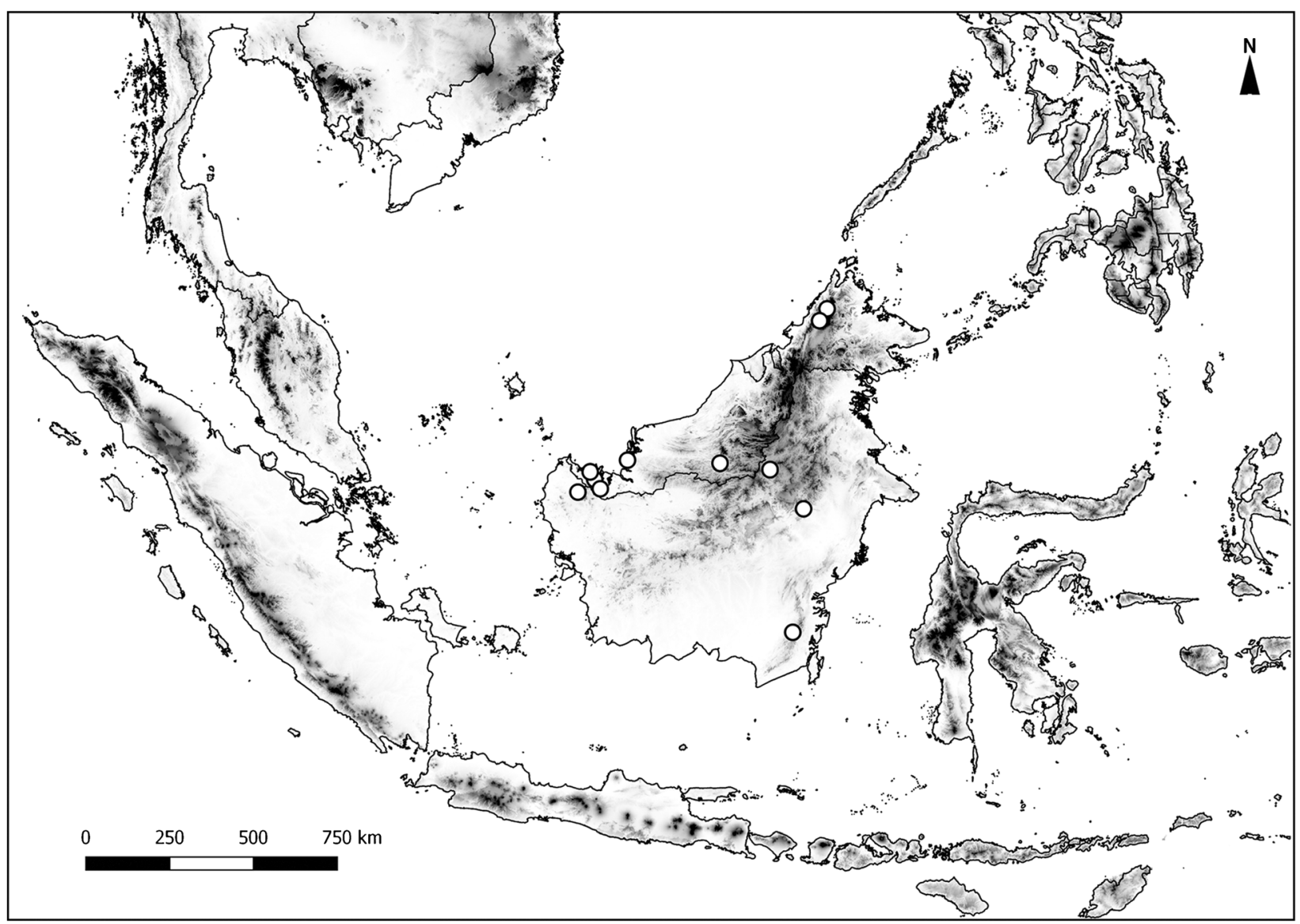

Map 3. Distribution of Korthalsia cheb. Dots are point localities of specimens examined.

green above, glabrous, with caducous, whitish indumentum on undersurface, distal margin conspicuously praemorse, transverse veinlets conspicuous, moderately closely spaced, $1.0-1.5 \mathrm{~mm}$. Inflorescence lax, $64.3-150 \mathrm{~cm}$ long including c. $19.2 \mathrm{~cm}$ peduncle, branched to $(1-) 2$ orders; prophyll $13.2-15.2 \times 1.2-2.0 \mathrm{~cm}$; rachis bracts up to $5.7-7.0 \times 2.0-2.7 \mathrm{~cm}$, tightly sheathing, with caducous, dark brown indumentum; primary branches $1-4,6.5$ $8.5 \mathrm{~cm}$ apart; proximal primary branch $18.0-29.5 \mathrm{~cm}$ long, with up to $1-4$ rachillae; rachillae $14.5-26.2 \mathrm{~cm}$ long and slender including $0.7-4.2 \mathrm{~cm}$ visible stalk, 0.7 $1.0 \mathrm{~cm}$ wide, densely hairy between rachilla bracts, orangebrown tomentose. Flower not seen. Fruit ovoid, $1.9-2.7 \times$ $1.2-1.9 \mathrm{~cm}$ covered in $16-21$ vertical rows of reddishbrown scales. Seed $1.7 \times 1.0 \mathrm{~cm}$; endosperm ruminate. (Figs 1A, 2A, 3A, 4, 8).

DISTRIBUTION. Peninsular Malaysia, Borneo (Brunei, Kalimantan, Sabah and Sarawak), Singapore, Sumatra (Map 4).

SPECIMENS EXAMINED. SUMATRA. INDONESIA. SUMATRA UTARA: Silo Maradja, Asahan, in the vicinity of Taloen Djoring, [3 $\left.{ }^{\circ} 8^{\prime} \mathrm{N} 99^{\circ} 10^{\prime} \mathrm{E}\right]$, Dec. 1927, Toroes 64 (K!); RIAU: Bukit Suligi, $200 \mathrm{~m}$ elev., [0 $\left.24^{\prime} \mathrm{N} 100^{\circ} 42^{\prime} \mathrm{E}\right]$, Aug. 1986, Sutisna 081 (K!); JAMBI: Batang
Tebopandak, nearest city Muara Bungo, 300 m elev., Sept. 1993, Trichon 796 (K!); BENGKULU: Muara Aman, Ketenong, $675 \mathrm{~m}$ elev., [3 ${ }^{\circ} 49^{\prime} \mathrm{S}$ 102 ${ }^{\circ} 43^{\prime} \mathrm{E}$ ], 18 Dec. 1984, Uhaedi 009 (K!); SUMATRA SELATAN: Palembang, De Vriese (L). MALAY PENINSULA. MALAYSIA. JOHOR: Muar, Ma' Okil Forest Reserve, Cpt. 218, valley bottom, $50 \mathrm{~m}$ elev., [409'N 10319'E], 7 June 1977, Dransfield 5019 (K!, KEP!). PAHANG: Maran, Cehabu, near Gun Tung Kat, N of Sori Jaya, 17 July 1987, Gianno 119 (A, BISH, BO, K!, KEP!, KLU, L, LAE, P, PNH, SAN, SAR, SING, TI, TNS, UC, US); Pekan, Ulu Jeram, 76.2 m elev., 2 July 1987, Gianno 79 (A, BISH, BO, K!, KEP!, KLU, L, LAE, P, PNH, SAN, SAR, SING, TI, TNS, UC, US); Kuantan, Bukit Ibam, [3 $10^{\circ} \mathrm{N}$ $102^{\circ} 58^{\prime} \mathrm{E}$ ], 30 May 1987, Gianno 36 (A, BISH, BO, K!, KEP!, KLU, L, LAE, P, PNH, SAN, SAR, SING, TI, TNS, UC, US). PERAK: Taiping, Maxwell's Hill, 975.36 m elev., [4 $4^{\circ} 52^{\prime} \mathrm{N} 100^{\circ} 47^{\prime} \mathrm{E}$ ], 9 March 1924, Burkill 12787 (K); Bota, Universiti Teknologi Mara, Bandar Seri Iskandar, [4 $\left.{ }^{\circ} 21^{\prime} \mathrm{N} 100^{\circ} 57^{\prime} \mathrm{E}\right]$, 9 Jan. 2007, Chan FRI 49263 (K!, KEP!). TERENGGANU: Kemaman, Bukit Kajang, 14 Nov. 1935, Corner 30467 (K, SING); Dungun, Jerangau Dalam, [4 $\left.4^{\circ} 49^{\prime} \mathrm{N} 103^{\circ} 11^{\prime} \mathrm{E}\right], 18$ Nov. 2014, Shahimi 9 (K!, KEP!). SINGAPORE: Herbarium Botanic Gardens, 15 June 1949, Furtado 37946 

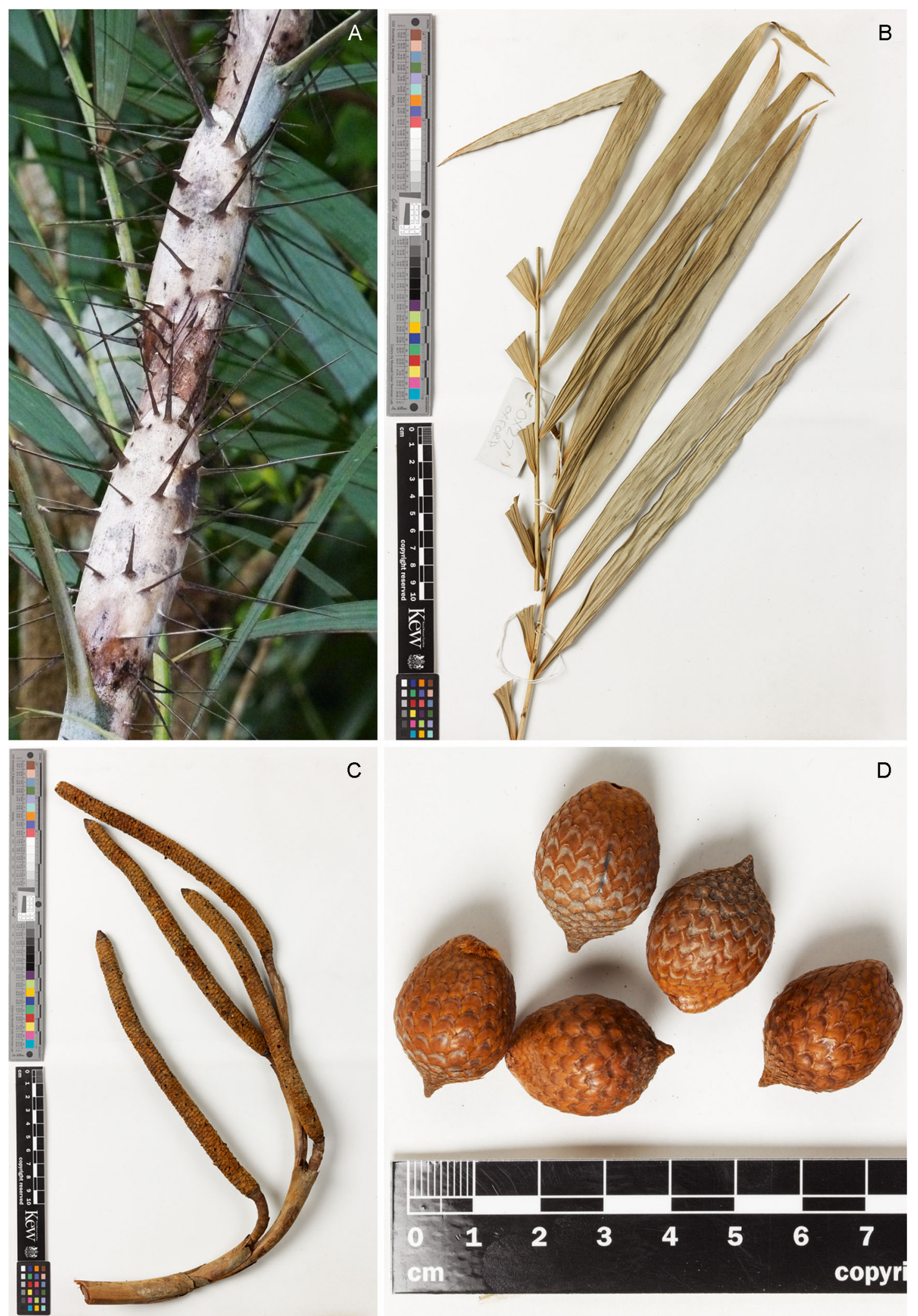

C
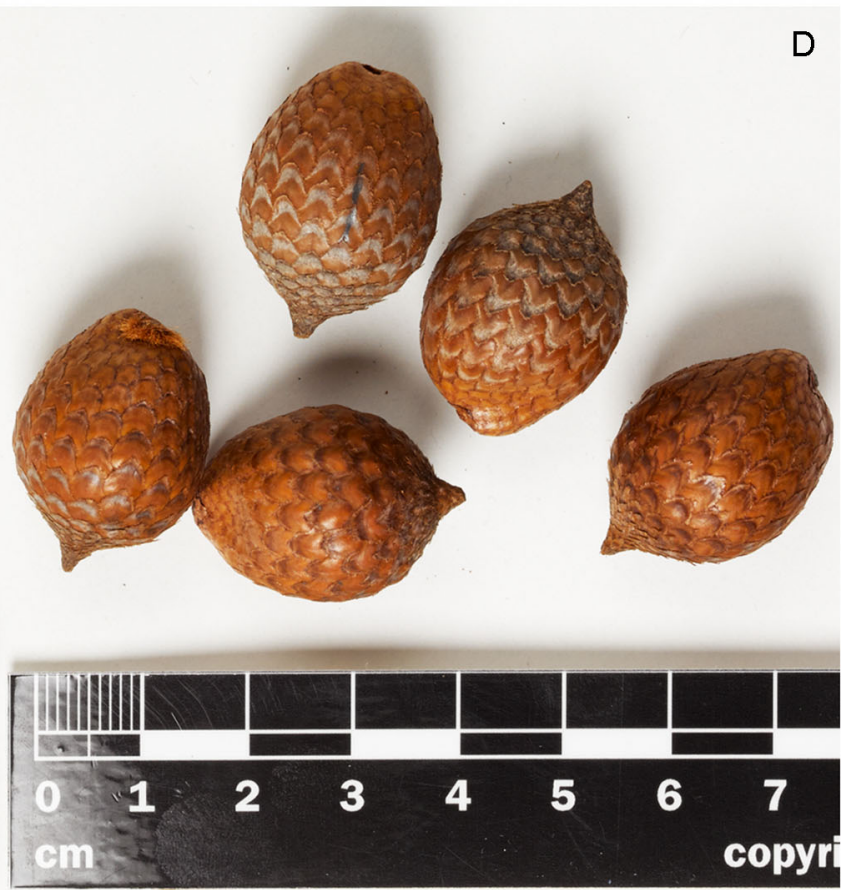

Fig. 8. Korthalsia echinometra. A ocrea; B leaflets (Bunker 27); C inflorescence (Dransfield 2828); D fruit (Dransfield 2828). 


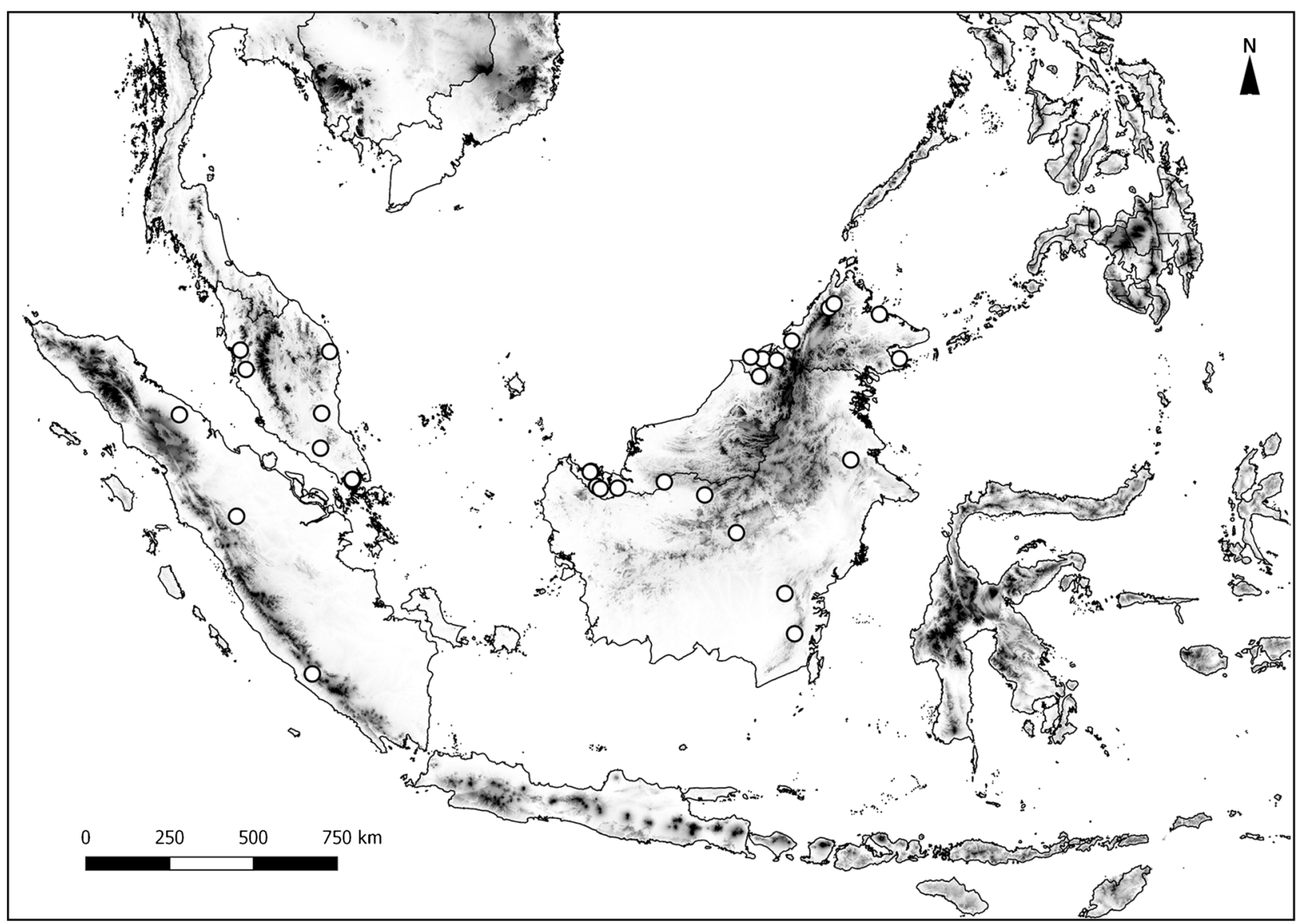

Map 4. Distribution of Korthalsia echinometra. Dots are point localities of specimens examined.

(AA, BH, BO, K!, L, LAE, KEP, PAR, PNH, SAR, SING!); Chau Chu Raug, 10 Dec. 1892, Ridley 3521 (K!); Nee Soon jungle, Thomson Road, bin Rani 27 (K, SING); Nee Soon Swamp Forest, [1 ${ }^{\circ} 24^{\prime} \mathrm{N} 103^{\circ} 48^{\prime} \mathrm{E}$, Rajasegar 18 (K!); MacRitchie Forest, $39 \mathrm{~m}$ elev., [1 ${ }^{\circ} 21^{\prime} \mathrm{N} 103^{\circ} 48^{\prime} \mathrm{E}$ ], 9 Dec. 2014, Shahimi 23 (K!, SING!). BORNEO. BRUNEI. BELAIT: along Sungai Ingei, [4 ${ }^{\circ} 10^{\prime} \mathrm{N} 114^{\circ} 43 ' \mathrm{E}$ ], 5 Jan. 1989, Wong s.n. (K!). TUTONG: Bukit Ladan Forest Reserve, Compartment 69, [4³8'N 11448'E], 13 April 1993, Ibrahim 15126 (K!); Sungai Liang, Sungai Liang Arboretum Reserve, [441'N 114²9'E], Wong 143 (K!). TEMBURONG: Batu Apoi, Ridge running $\mathrm{W}$ of landing place, $250 \mathrm{~m}$ elev.,

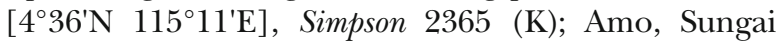
Temburong at Kampung Belalong, ridge $\mathrm{W}$ of river, 150 m elev., [4 $\left.36^{\prime} \mathrm{N} 115^{\circ} 11^{\prime} \mathrm{E}\right], 25$ June 1989, Dransfield 6721 (K!). INDONESIA. KALIMANTAN UTARA: Northern part of island Nunukan, NE Borneo (Kalimantan), km 3 from the coast, 6 Nov. 1953, Meijer 2054 (K!). KALIMANTAN BARAT: Mendalam R., Betung Kerihun National Park, $200 \mathrm{~m}$ elev., [0 59'N $113^{\circ} 15^{\prime} \mathrm{E}$ ], 1 Aug. 2001, Watanabe 14 (BO, K!); Sanggau, near village of Bedigong, 2 Feb. 1996, Graefen 2 (K!). KALIMANTAN SELATAN: District Tabalong, Upper Tabalong Area, PT Aya Yayang Indonesia Concession,
Misim, $50 \mathrm{~m}$ elev., [ $1^{\circ} 40^{\prime} \mathrm{S} 115^{\circ} 24 ' \mathrm{E}$ ], 5 Aug. 2000, Mogea 7403 (BO, K!, L, NY, WAN); P.T. Yayang Tanjung, 200 m elev., 22 Aug. 1985, Auggana 047 (K); Barabai, Pergunungan Meratus, foothills of Gunung Besar, 500 m elev., [2 $45^{\prime} \mathrm{S} 115^{\circ} 40$ 'E], 18 Oct. 1972, Dransfield 2828 (BH, K!, L). KALIMANTAN TENGAH: near Rekut base camp, $250 \mathrm{~m}$ elev., [0²'S 1146'E], 11 April 1992, Awmack 296 (K!). KALIMANTAN TIMUR: Berau, RKT 9293, Inhutani I concession, $250 \mathrm{~m}$ elev., 8 Oct. 1991, Stockdale 143 (K!); Berau, near km 37 Camp, Inhutani I, 80 m elev., [ $1^{\circ} 55^{\prime} \mathrm{N} 117^{\circ} 10^{\prime} \mathrm{E}$ ], 9 Aug. 2003, Watanabe 60 (K!); between Sinara and Entang Rivers on [floodplain] of Sangatta River, Kutai Reserve, 200 m elev., 31 Oct. 1991, Stockdale 87 (K!). MALAYSIA. SABAH: Sandakan, Kabili-Sepilok Forest Reverse, [ [5 $50^{\circ} \mathrm{N} 117^{\circ} 56^{\prime} \mathrm{E}$ ], 12 June 1937, Enggoh 7432 (K!); Semporna, Mapat Reserve, Timbun Mata Forest Reserve, 1 Aug. 1937, Puasa 7404 (K!); Kampung Kauluan, [60'N 116³5'E], 9 May 1994, Sambuling 169 (K!); Ranau, Kampung Takutan, [6 ${ }^{\circ} 7^{\prime} \mathrm{N}$ 116²3'E], 29 March 1995, Tadong 179 (K!); Ranau, Kampung Poring, Minintaiku Forest Reserve, 4 May 1994, Sambuling 144 (K!); Kampung Poring, on the hill top, 4 Jan. 1994, Sambuling 45 (K!); Kampung Poring, 9 Sept. 1992, Giking 22 (K!); Kampung Poring, 10 Feb. 1994, Sambuling 82 (K!); Kampung Tensungoi, 
[57'N 115³5'E], 2 June 1994, Sambuling 202 (K!); Mile 55, Sandakan-Telupid, $100 \mathrm{~m}$ elev., 26 Oct. 1979, Dransfield et al. 5818 (K!, SAF, SAR). SARAWAK: Kuching, Gunung Serapi, Kubah National Park, forest trail, 274.32 m elev., [1 ${ }^{\circ} 36^{\prime} \mathrm{N} 110^{\circ} 12^{\prime} \mathrm{E}$ ], 7 Feb. 1995, Baker 509 (K!, KEP!); Kuching, 1st Division, Kampung Semedang, 24th Mile Penrissen Road, 11 Aug. 1985, Saigol 15 (K!); Gunung Matang, 1 June 1866, [1 $\left.{ }^{\circ} 36^{\prime} \mathrm{N} 110^{\circ} 10^{\prime} \mathrm{E}\right]$, Beccari PB 1935 (FI!); Gunung Matang, [1 $\left.{ }^{\circ} 36^{\prime} \mathrm{N} 110^{\circ} 10^{\prime} \mathrm{E}\right], 1$ June 1866, Beccari PB 1918 (FI!); 1st Division, Kampung Gayu, $250 \mathrm{~m}$ elev., [1 ${ }^{\circ} 13^{\prime} \mathrm{N} 110^{\circ} 21$ 'E], 15 July 1993, Bunker 27 (K!); 1st Division, Kampung Kakeng, 200 m elev., [1 ${ }^{\circ} 9^{\prime} \mathrm{N} 110^{\circ} 27^{\prime} \mathrm{E}$ ], 20 July 1993, Bunker OX 39 (K!); Kapit, 7th Division, 8 km Logging Camp, Nanga Mujong, 28 Aug. 1985, Saigol 25 (K!); Lubok Antu, 2nd Division, Delok R., on slope in open secondary forest near Nanga Sumpa, $250 \mathrm{~m}$ elev., [1 $\left.{ }^{\circ} 20^{\prime} \mathrm{N} 112^{\circ} 10^{\prime} \mathrm{E}\right], 5$ March 1993, Christensen 1211 (K!); Simunjan, 1st/2nd Division Border Gunung Buri, near summit, $700 \mathrm{~m}$ elev., $\left[1^{\circ} 10^{\prime} \mathrm{N}\right.$ $110^{\circ} 55^{\prime} \mathrm{E}$ ], 22 May 1981, Dransfield et al. 6115 (K!, L, NY); Baram, on hill behind house, Long Selatong Ulu, 228.6 m elev., 15 July 1977, Chung 2768 (K!); Belaga, 3rd Division, Sungai Masoh, rumah Nyaving, Kuala Linau, Ulu Belaga, 200 m elev., 9 Aug. 1975, Dransfield 4688 (K!, KEP!, L).

HABITAT. Lowland and hill dipterocarp forest up to $1000 \mathrm{~m}$ above sea level.

VERNACULAR NAMES. Wi wisto (Iban), Wee tujah (Kayan), Owei lia (Murut), Uwai kapot alot (Kenyah), Paikat talusuk (Dayak lawangan), Rotan keruk (Iban), Rotan udang (Jakun), Rotan seru (Iban), Rotan lia (Dusun), Pipit gading (Dusun), Wi serok (Iban), Rotan semut udang (Dayak), Semeii (Bidayuh).

USES. Cane used to make basket frames, weaving handicrafts and also to tie planks on dugouts to raise the side wall of canoes. Sap can be drunk for fever.

NOTES. Korthalsia echinometra is one of the commonest species of the genus Korthalsia. It is a very distinctive species, yet from a distance, it can be mistaken for other rattan genera because of its narrow lanceolate leaflets. Its large inflated ocrea with long slender spines and narrowly lanceolate leaflets are diagnostic. The ocreas of $K$. echinometra are reported to be inhabited by ants of the species Camponotus contractus (Bequaert \& Wheeler 1922) and Iridomyrmex species (Chan et al. 2012). One of us (WJB) has observed evidence of ants modifying the ocreas of $K$. echinometra by biting fibres in the ocrea wall, which then result in minute slits a few millimetres long opening in the ocrea, presumably altering the microclimate within the ocrea.

4. Korthalsia furcata Becc. (Beccari 1918: 120). Type: Indonesia, West Kalimantan, Sungai Kenepai, Hallier 2034 (holotype BO; isotype FI!).
Slender, clustering rattan climbing to $20 \mathrm{~m}$. Stem with sheaths $4-6 \mathrm{~mm}$ diam., without sheaths $2-3 \mathrm{~mm}$ diam.; internodes c. 9 cm. Leaf c. $0.6 \mathrm{~m}$ long including cirrus and petiole; sheath brown, with caducous, brown-coloured indumentum, sheath armed with scattered abundant brown triangular spines, spines 1 - $3 \mathrm{~mm}$ long, c. $1 \mathrm{~mm}$ wide; ocrea $20-25 \times 10-11$ $\mathrm{mm}$, small, almost rounded and start inflated above the petiole, clasping the stem, tough, brownish, armed with c. $2 \mathrm{~mm}$ long tiny triangular spines, ant present; cirrus c. $0.28 \mathrm{~m}$, armed with recurved grapnel spines organised in whorls; petiole c. $2 \mathrm{~cm}$, c. $4 \mathrm{~mm}$ wide and c. $2 \mathrm{~mm}$ thick at base, flattened adaxially, abaxially rounded, with caducous, indumentum as sheath, sparsely armed with spines; rachis $3.5-4.0 \mathrm{~cm}$ in length, armed with recurved grapnel spines; usually only one leaflet each side of rachis, regularly arranged, furcate (bilobed), mid-leaf leaflets 33.8 $36.7 \times 4.2-4.6 \mathrm{~cm}$, leaflet dull green above, glabrous, caducous, grey-whitish indumentum on undersurface, with inconspicuously praemorse margin, transverse veinlets moderately conspicuous, fine and closely spaced, c. $0.5 \mathrm{~mm}$. Inflorescence lax, c. $35 \mathrm{~cm}$ long, branched to 2 orders; rachis bracts up to $3.0-7.7 \times 0.7$ - $1.5 \mathrm{~cm}$, splitting, with caducous, dark brown indumentum; primary branches $2,3.5-8.3 \mathrm{~cm}$ apart; proximal primary branch $14.3 \mathrm{~cm}$ long, with up to 2 rachillae; rachillae 4.7 - $17.2 \mathrm{~cm}$ long and slender including $0.6-1.2 \mathrm{~cm}$ visible stalk, $0.6-0.7 \mathrm{~cm}$ wide, densely hairy between rachilla bracts. Flower not seen. Fruit mature fruit not seen. Seed not seen (Fig. 9).

DISTRIBUTION. Borneo (1st Division at Sarawak, Malaysia and West Kalimantan, Indonesia) (Map 5).

SPECIMENS EXAMINED. BORNEO. INDONESIA. KALIMANTAN BARAT: Sungai Kenepai, [0 $\left.35^{\prime} \mathrm{N} 111^{\circ} 56^{\prime} \mathrm{E}\right]$, Hallier 2034 (BO, FI!). MALAYSIA. SARAWAK: Serian, 1st Division, Sabal Tapang Forest Reserve, Block 14, 350 m elev., [1 ${ }^{\circ} 0^{\prime} \mathrm{N} 110^{\circ} 51$ 'E], 18 May 1981, Dransfield et al. 6074 (B, K!, L, NY); Kuching, Sungai Kopak, Ulu Samunsam, $200 \mathrm{~m}$ elev., [1 ${ }^{\circ} 49^{\prime} \mathrm{N}$ 106 $\left.37^{\prime} \mathrm{E}\right], 21$ Aug. 1998, Rantai E゚ Lai 74544 (K!, KEP, L).

HABITAT. In valley bottom in transition between hill dipterocarp forest and Kerangas at $350 \mathrm{~m}$ altitude.

VERNACULAR NAME. Not recorded.

USES. Not recorded.

NOTES. Korthalsia furcata is very similar to $K$. rostrata but can be readily distinguished by the leaf, which has only two leaflets that appear forked. It is an extremely rare and poorly known species that is likely to be threatened with extinction.

5. Korthalsia furtadoana J.Dransf. (Dransfield 1981: 185). Type: Malaysia, Sabah, 16 Oct. 1979, Dransfield et al. JD 5763 (holotype K!; isotype SAN, SAR). 

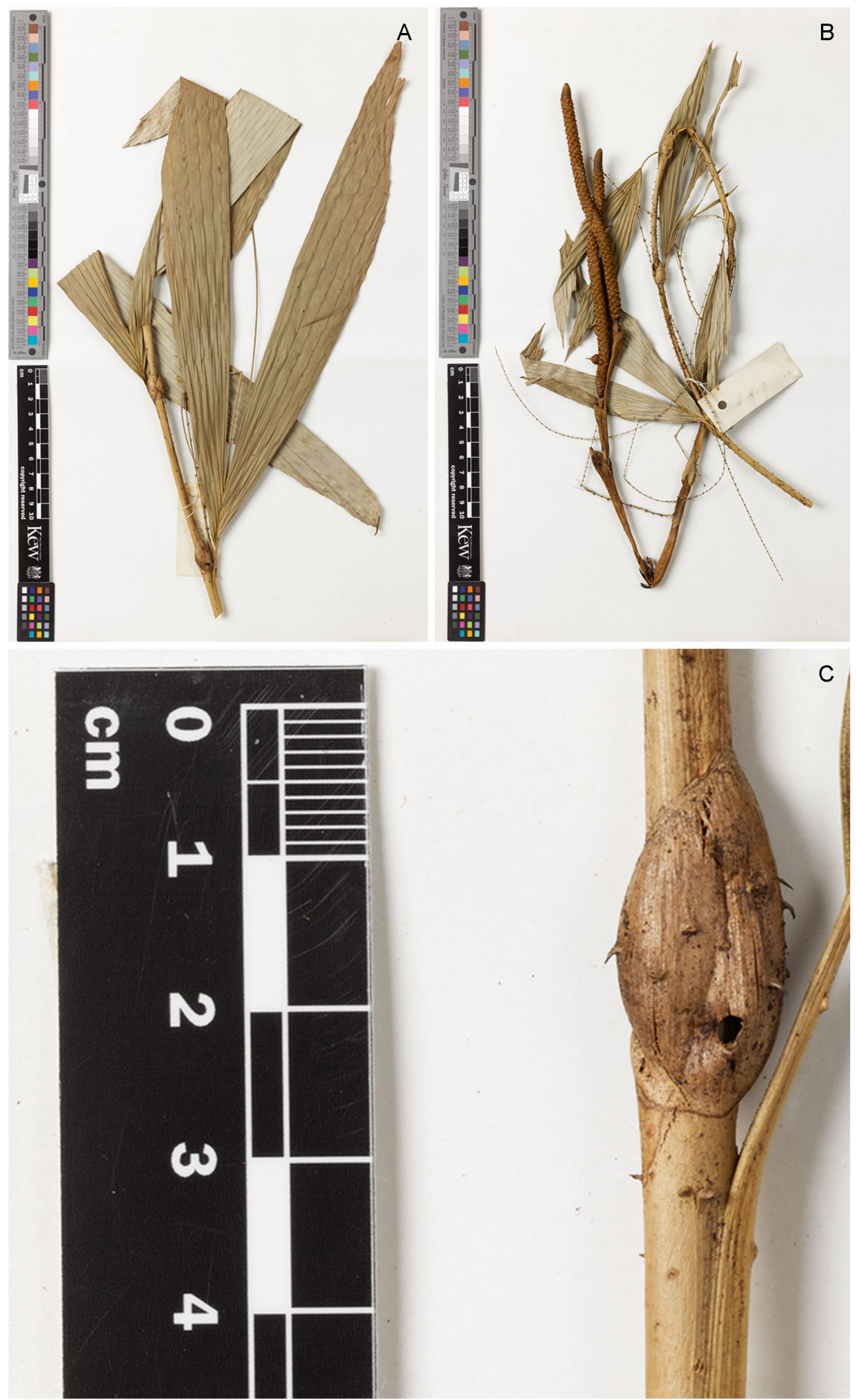

Fig. 9. Korthalsia furcata. A leaflets (Dransfield et al. 6074); B inflorescence (Dransfield et al. 6074); C ocrea (Dransfield et al. 6074). 


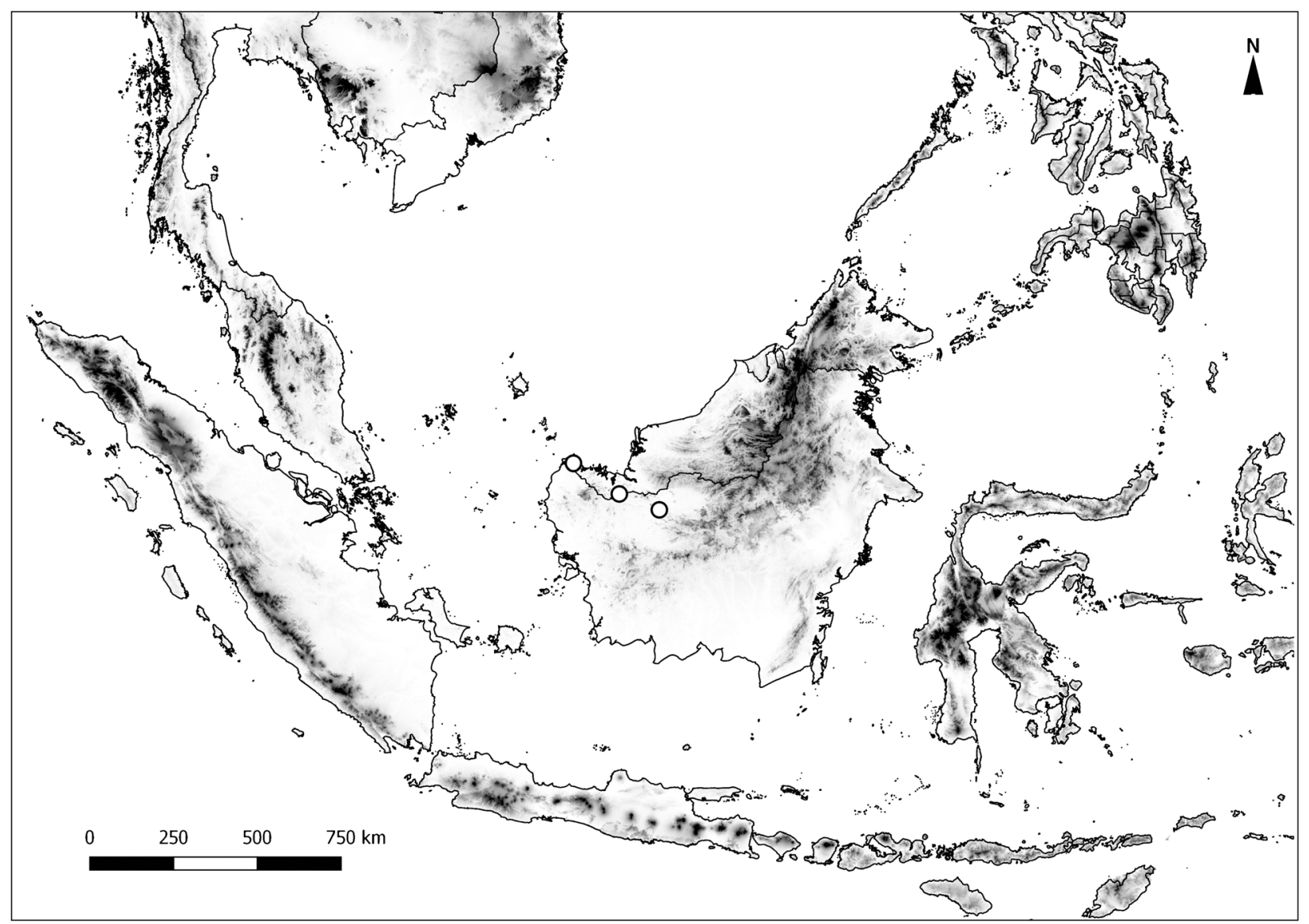

Map 5. Distribution of Korthalsia furcata. Dots are point localities of specimens examined.

Slender, clustering rattan climbing to $20 \mathrm{~m}$. Stem with sheaths $8-11 \mathrm{~mm}$ diam., without sheaths $5-8 \mathrm{~mm}$ diam.; internodes $7-12 \mathrm{~cm}$. Leaf $0.60-0.88 \mathrm{~m}$ long including cirrus and petiole; sheath green, with indumentum brown in colour, sheath armed with scattered abundant black triangular spines, spines 1 $6 \mathrm{~mm}$ long, $1-2 \mathrm{~mm}$ wide; ocrea $25-83 \times 10-15$ $\mathrm{mm}$, conspicuous, tightly sheathing above the petiole and then elongate inflated, clasping the stem, tough, brown, armed with $1-6 \mathrm{~mm}$ long, triangular black spines, numerous ants within the ocrea, entry hole made by ants visible; cirrus $0.3-0.7 \mathrm{~m}$, armed with recurved grapnel spines organised in whorls; petiole $25-70 \mathrm{~mm}, 5-7 \mathrm{~mm}$ wide and $2-3 \mathrm{~mm}$ thick at base, flattened adaxially, abaxially rounded, with a few spots of brown indumentum, armed with spines as sheath and reflexed grapnel spines; rachis $0.27-0.43$ $\mathrm{m}$, armed with recurved grapnel spines; 5 - 6 leaflets each side of rachis, regularly arranged, narrowly rhomboid, mid-leaf leaflets $9.0-19.7 \times 4.4-6.6 \mathrm{~cm}$, leaflet dull to dark green above, glabrous, lacking indumentum on undersurface, with inconspicuously praemorse margin, transverse veinlets conspicuous, fine and closely spaced, c. $0.5 \mathrm{~mm}$. Inflorescence lax, 26.5 - $100 \mathrm{~cm}$ long including c. $10 \mathrm{~cm}$ peduncle, branched to 2 orders; prophyll $1.5-6.5 \times 0.5-0.7 \mathrm{~cm}$; rachis bract up to $3.5-5.9 \times 1.3-3.3 \mathrm{~cm}$, tightly sheathing and splitting, caducous, brown indumentum with several tiny spines; primary branches $1-2,9.5-$ $13.5 \mathrm{~cm}$ apart; proximal primary branch 13.5 $19.5 \mathrm{~cm}$ long, with up to $1-3$ rachillae; rachillae $12.0-19.8 \mathrm{~cm}$ long and congested, stalk not visible, $0.7-1.4 \mathrm{~cm}$ wide, densely hairy between rachilla bracts. Flower $8 \times 4 \mathrm{~mm}$ in the bud. Fruit ovoid, $1.3-2.2$ $\times 0.8-1.2 \mathrm{~cm}$ covered in $15-23$ vertical rows of yellow-brownish scales. Seed $1.2 \times 0.8 \mathrm{~cm}$; endosperm ruminate (Fig. 10).

DISTRIBUtION. Borneo (Brunei, Sabah, Central, East and South Kalimantan) (Map 6).

SPECIMENS EXAMINED. BORNEO. BRUNEI. TEMBURONG: Amo, beside the Belalong R., [4 ${ }^{\circ} 37^{\prime} \mathrm{N} 115^{\circ} 6^{\prime} \mathrm{E}$ ], Wong 1326 (K!); Batu Apoi, Bukit Belitun, Sungai Sinupoi, $\mathrm{S}$ of village, $40 \mathrm{~m}$ elev., [4 $\left.36^{\prime} \mathrm{N} 115^{\circ} 11^{\prime} \mathrm{E}\right], 21$ Jan. 1994, Dransfield 7477 (K!). TUTONG: Lamunin, Compartment 1, $30 \mathrm{~m}$ elev., [4 $41^{\prime} \mathrm{N} 114^{\circ} 43^{\prime} \mathrm{E}$ ], Nov. 1990, Dransfield et al. 6875 (K!). INDONESIA. KALIMANTAN SELATAN: Tabaling, Upper Tabalong Area, PT Aya Yayang Indonesia Concession, Kebun Bibit, km 54, Sungai Papun, 200 m elev., 6 Aug. 2000, 

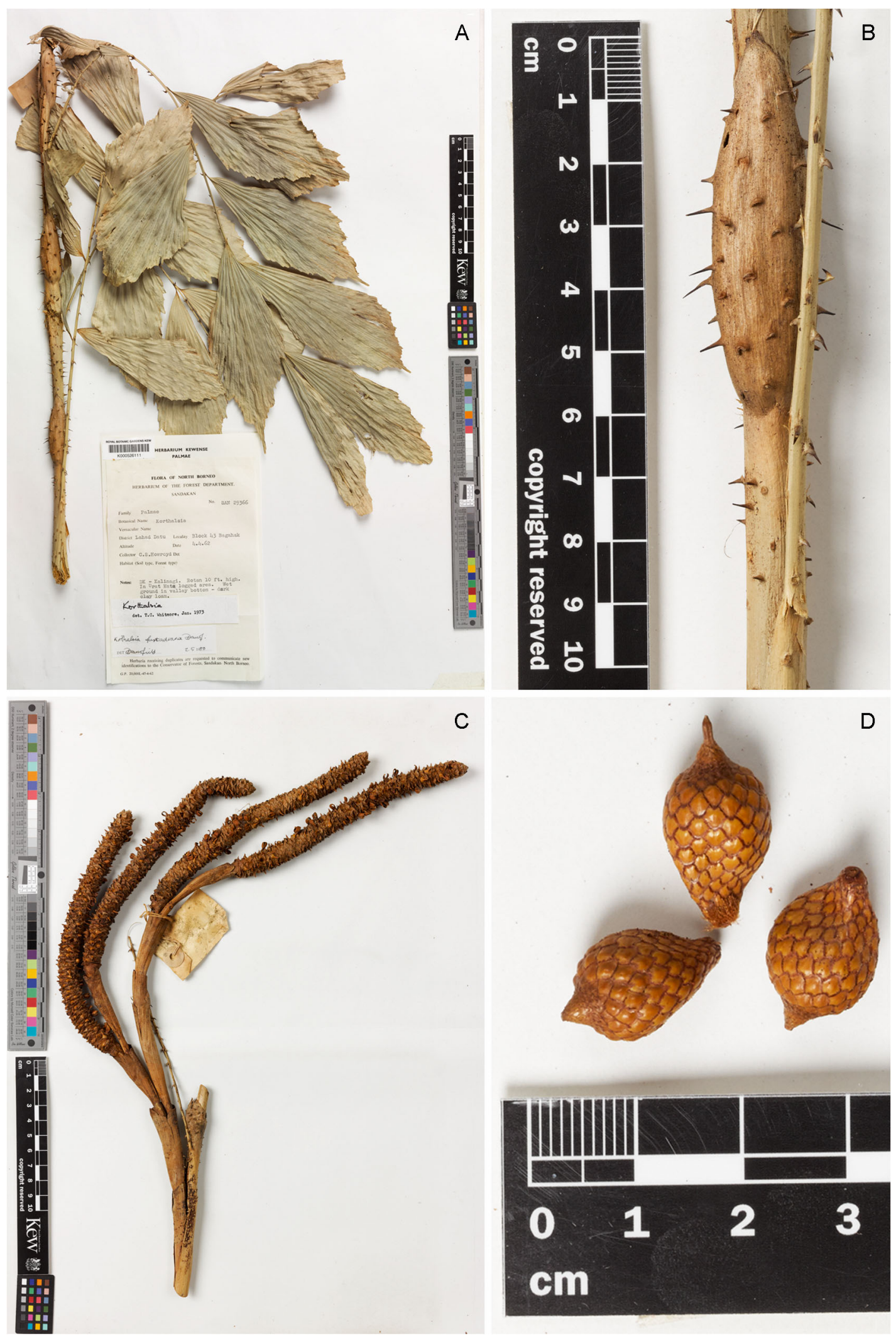

Fig. 10. Korthalsia furtadoana. A leaflets (Howroyd SAN29366); B ocrea (Howroyd SAN29366); C inflorescence (Howroyd SAN29366); D fruit (Howroyd SAN29366). 


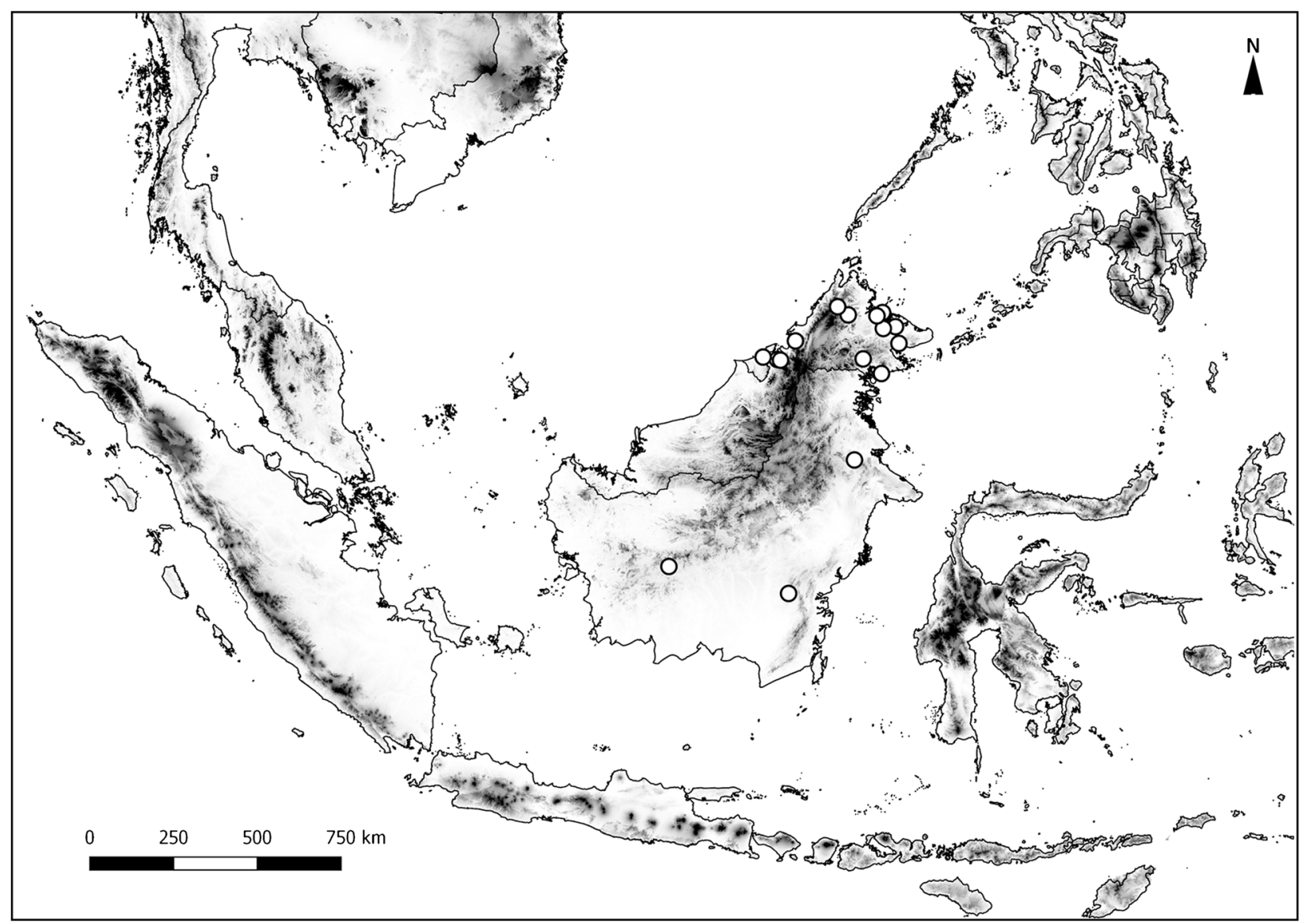

Map 6. Distribution of Korthalsia furtadoana. Dots are point localities of specimens examined.

Mogea 7431 (BO, K!, L, NY, WAN); Tabalong, Upper Tabalong Area, PT Aya Yayang Indonesia Concession, cutting block 1999/2000 near km 54 Kebun Bibit, 400 m elev., 12 Aug. 2000, Mogea 7436 (BO, K!, L, NY, WAN); Upper Tabalong Area, PT Aya Yayang Indonesia Concession, Misim, $50 \mathrm{~m}$ elev., [1 ${ }^{\circ} 40^{\prime} \mathrm{S}$ $115^{\circ} 24 ' \mathrm{E}$ ], 5 Aug. 2000, Mogea 7408 (BO, K!, L, NY, WAN); PT Yayang Tanjung, 20 Aug. 1985, Anggana 039 (K). KALIMANTAN TENGAH: Bukit Raya, [056'S $\left.112^{\circ} 11^{\prime} \mathrm{E}\right]$, Leighton 376 (K). KALIMANTAN TIMUR: Berau, near km 37 Camp, Inhutani I, 80 m elev., [1 ${ }^{\circ} 55^{\prime} \mathrm{N} 117^{\circ} 10^{\prime} \mathrm{E}$ ], 9 Aug. 2003, Watanabe 59 (K!, $\mathrm{BO}) ; 7 \mathrm{~km}$ from Eheng (village), Barong Tongkok (district) near Kelian, upper Mahakan, 10 Nov. 1991, Stockdale 101 (K!); Lingau, 2 km from Prampus, P.T. KEM cencession near Kelian, upper Mahakan R., 11 Nov. 1991, Stockdale 104 (K!); near Sangatta R., Kutai Reserve, halfway between "Prevab" camp and "Mentoko" camp, 20 m elev., 30 Oct. 1991, Stockdale 78 (K!); Mentoko Camp, Kutai Reserve, 50 m elev., 31 Oct. 1991, Stockdale 85 (K!). MALAYSIA. SABAH: Kalabakan, Hap Seng logged area, km 12, 12 June 1982, Krispinus 94884 (K); Kota Kinabatangan, Hutan Simpan Pin-Supu, $80 \mathrm{~m}$ elev., [5 $27^{\prime} \mathrm{N}$ 11756'E], 2 April 1992, Dewol SAN 132477 (K!,
SAN); Lahad Datu, Forest Distr. Lahad Datu, [5 ${ }^{\circ} 2^{\prime} \mathrm{N}$ $118^{\circ} 21$ 'E], 5 Nov. 1949, Cuadra 2498 (K!); Lahad Datu, Block 43 Bagahak, 4 April 1962, Howroyd SAN 29366 (K!); Lahad Datu, Cpt. 54 Bakapit, Kennedy Bay, 457.2 m elev., June 1962, Burgess SAN 30782 (K!); Nabawan, Mile 21ํ2 Rahsna Road, 19 Jan. 1978, Abas SAN 85854 (K!, L, SAR); Pinangah, Ulu Sg. Melikop, 30 Aug. 1984, Leopold SAN 64584 (K!); Ranau, Ulu Tungud Forest Reserve, Gunung Monkobo Expedition, $471 \mathrm{~m}$ elev., [5 $49^{\prime} \mathrm{N}$ $117^{\circ} 0^{\prime} \mathrm{E}$ ], 25 July 2005, Saw SAN 146691 (K!, KEP!, L, SAN, SAR); Ranau, Kampung Poring, [6 ${ }^{\circ} 2^{\prime} \mathrm{N}$ $116^{\circ} 42^{\prime} \mathrm{E}$ ], 11 Feb. 1994, Sambuling 93 (K!); Sandakan, Ulu Dusun Agriculture station, [5 47' N $117^{\circ} 46^{\prime} \mathrm{E}$ ], 16 Oct. 1979, Dransfield et al. JD 5763 (K!, SAN, SAR); Sandakan, Sungai Menenggul, [5 $30^{\prime} \mathrm{N} 118^{\circ} 16^{\prime} \mathrm{E}$ ], 27 Feb. 1985, Amin SAN 68050 (K!, KEP!); Sandakan, Sepilok Forest Reserve, Forest Distr. Elopura, [5 $52^{\prime} \mathrm{N} 117^{\circ} 55^{\prime} \mathrm{E}$ ], 24 Sept. 1949, Kadir 2643 (K!, KEP!); Sandakan, Sepilok Forest Reserve, $50 \mathrm{~m}$ elev., [5 $\left.52^{\prime} \mathrm{N} 117^{\circ} 55^{\prime} \mathrm{E}\right], 28$ Feb. 1995, Baker 553 (K!); Sandakan, Kabili-Sepilok Forest Reserve, [5 $50^{\prime} \mathrm{N} 117^{\circ} 56^{\prime} \mathrm{E}$ ], 25 July 1937, Matusop 7427 (K!); Sandakan, Ulu Dusun Agriculture station, 7 m elev., 16 Sept. 1979, Dransfield et al. 5763 
(K!, L, SAF, SAR); Sandakan, Subak camp, Jesselton Distr., 19 Dec. 1962, Madani SAN 33215 (K!); Tawau, St Lucia, Ranggu Mile 10 3/4, state forest, 16 Sept. 1949, Cuadra 2377 (KEP); Tawau, Luasong Forest Reserve, logging area, [4 $\left.{ }^{\circ} 38^{\prime} \mathrm{N} 117^{\circ} 24^{\prime} \mathrm{E}\right], 16$ Aug. 1977, Fedilis SAN 87333 (K!, KEP!, L, SAR, SING!); Tawau, mile 10 ó State Forest, Forest Distr. St. Lucia, 16 Sept. 1949, Lenedia 2377 (K!); Tawau, Elphinstone Province, [4 ${ }^{\circ} 14^{\prime} \mathrm{N} 117^{\circ} 53$ 'E], Oct. 1922, Elmer 20476 (K!); Tenom, Paling-paling Hills, Lagud, 300 m elev., 4 Sept. 1979, Dransfield et al. 5591 (K!, L, SAF, SAR); Danum Valley, Ulu Segama, plot 2, W trail from field station, $150 \mathrm{~m}$ elev., 9 March 1987, Argent 291987 (E!, K!); BetoTan, 15.24 m elev., 16 May 1933, Castro 3201 (K); Kampung Wolit, 1/2 km from house to E, 29 Aug. 1997, Jinuan 24 (K); Ulu Segama, W trail, 200 m elev., 16 Feb. 1986, Dransfield 6260 (K); Kampung Tensungoi, [57'N 115³5'E], 2 June 1994, Sambuling 198 (K!).

HABITAT. Lowland dipterocarp forest.

VERNACULAR NAME. Not recorded.

USES. Not recorded.

NOTES. See notes under Korthalsia rostrata for discussion of this species. Korthalsia furtadoana ocreas are inhabited by ants of the genera Camponotus and Crematogaster (Edwards et al. 2010).

6. Korthalsia hispida Becc. (Beccari 1884: 71). Type: Indonesia, Sumatra, 1 Aug. 1878, Beccari PS 643 (holotype FI!: isotype $\mathrm{K}$ !).

Moderately robust, clustering rattan climbing to $20 \mathrm{~m}$. Stem with sheaths $11-25 \mathrm{~mm}$ diam., without sheaths 8 $10 \mathrm{~mm}$ diam.; internodes $10-20 \mathrm{~cm}$. Leaf $1.15-2.0 \mathrm{~m}$ long including cirrus and petiole; sheath bright green, with caducous, brown-coloured indumentum, sheath armed with scattered abundant, black, needle-like spines, spine 2 - $30 \mathrm{~mm}$ long, c. $1 \mathrm{~mm}$ wide; ocrea $18.5-30.0 \times$ $3.0-4.5 \mathrm{~cm}$, conspicuous, diverging from the angle at stem just above the petiole, the margins tending to inroll, papery, brown, armed with $3-30 \mathrm{~mm}$ long black spines and abundant chocolate spicules, abundant black fierce and very noise ants nested within the ocrea; cirrus $0.5-1.0$ $\mathrm{m}$, armed with recurved grapnel spines organised in whorls; petiole $10.0-35.0 \mathrm{~cm}, 10-20 \mathrm{~mm}$ wide and 3 $5 \mathrm{~mm}$ thick at base, flattened adaxially, abaxially rounded, with brown indumentum, sparsely armed with fine spines as sheath; rachis $0.30-1.07 \mathrm{~m}$, armed with recurved grapnel spines; $5-8$ leaflets each side of rachis, regularly arranged, rhomboid, mid-leaf leaflets 15.0 - $19.6 \times 5.0-$ $8.6 \mathrm{~cm}$, leaflet dull to light green above, glabrous, with caducous, whitish indumentum on undersurface, distal margin conspicuously praemorse, transverse veinlets moderately conspicuous and moderately spaced, 1.0 $1.5 \mathrm{~mm}$. Inflorescence lax to congested, $30.0-50.2 \mathrm{~cm}$ long including c. $45 \mathrm{~mm}$ peduncle, branched to (1 -) 2 orders; prophyll $4.5 \times 1.0 \mathrm{~cm}$, with caducous, dark brown indumentum with scattered tiny spines; rachis bracts up to $8.3-9.5 \times 2.6-3.4 \mathrm{~cm}$, conspicuous, inflated and splitting, with caducous, dark brown indumentum and densely covered with chocolate scales and black spicules; primary branches $1-4,4.0-6.6 \mathrm{~cm}$ apart; proximal primary branch $17.0-27.5 \mathrm{~cm}$ long, with up to $1-3$ rachillae; rachillae $11.5-22.3 \mathrm{~cm}$ long and slender, stalk not visible, $1.0-1.8 \mathrm{~cm}$ wide, lacking hairy between rachilla bracts. Flower $8-8.1 \times 3.3-3.7 \mathrm{~mm}$ in the bud. Fruit round to oblong, $1.8-2.3 \times 0.9-1.6 \mathrm{~cm}$ covered in $15-18$ vertical rows of reddish-brown scales. Seed $1.1 \times 0.8$ cm; endosperm homogeneous (Fig. 11).

DISTRIBUtION. Malay Peninsula, Borneo (Brunei, Sabah, Sarawak, East and South Kalimantan), Sumatra (Map 7).

SPECIMENS EXAMINED. SUMATRA. INDONESIA. SUMATRA BARAT: Padang, Ayer manicior (Ajer mantjoer), [0 $57^{\circ} \mathrm{S} 100^{\circ} 25^{\prime} \mathrm{E}$ ], Aug. 1878, Beccari 643 (K!). MALAY PENINSULA. MALAYSIA. JOHOR: Bekuk, Hutan Lipur Batang, $90 \mathrm{~m}$ elev., [2 $20^{\circ} \mathrm{N} 103^{\circ} 9^{\prime} \mathrm{E}$ ], 24 Nov. 2014, Shahimi 11 (K!, KEP!); Kota Tinggi, Panti Forest Reserve, 10 m elev., 10 June 1977, Dransfield 5035 (K!); Kota Tinggi, Gunong Panti, East Forest Reserve, $20 \mathrm{~m}$ elev., [1 ${ }^{\circ} 50^{\prime} \mathrm{N} 103^{\circ} 54^{\prime} \mathrm{E}$ ], 7 Jan. 1973, Dransfield 3037 (BH, BO, BRI, CAL, CANB, G, K!, KEP, L, LAE, MAN, NSW, NY, PAR, SAN, SAR, SING!, US); Labis, Labis Forest Reserve, Ulu Endau, Gunung Janing, valley bottom, $100 \mathrm{~m}$ elev., $\left[2^{\circ} 10^{\prime} \mathrm{N} 103^{\circ} 22^{\prime} \mathrm{E}\right], 16$ June 1977, Dransfield 5090 (K!). PAHANG: Kuantan, Galing Forest Reserve, $20 \mathrm{~m}$ elev., [3 ${ }^{\circ} 52^{\prime} \mathrm{N} 103^{\circ} 19^{\prime} \mathrm{E}$ ], 11 Aug. 1977, Dransfield 5227 (K!). BORNEO. BRUNEI. BELAIT: Labi, Teraja Longhouse, [4 $\left.{ }^{\circ} 23^{\prime} \mathrm{N} 114^{\circ} 26^{\prime} \mathrm{E}\right]$, Wong Ẽ Dransfield 540 (K!, SING!). TEMBURONG: Amo, Belalong R. bank near Field Studies Centre, E side, $300 \mathrm{~m}$ elev., [4³3'N 1159'E], 6 Sept. 1991, Stockdale 31 (K!); Batu Apoi, Kerangan Batu Semawat, WNW of village, $30 \mathrm{~m}$ elev., [4 $36^{\circ} \mathrm{N} 115^{\circ} 11 \mathrm{E}$ ], Dransfield et al. 7494 (K!); Batu Apoi National Park, Kuala Belalong Field Centre Temburong R., $50 \mathrm{~m}$ elev., [4 35'N $115^{\circ} 10^{\prime} \mathrm{E}$ ], 15 Feb. 1995, Baker 531 (BRUN, K!, UBD); South Temburong, above Kuala Belalong, $75 \mathrm{~m}$ elev., [430'N 15¹0'E], 21 Feb. 1991, Argent et al. 9168 (K!). TUTONG: Lamunin, between the rivers Batas and Tutong, [4 $41^{\prime} \mathrm{N} 114^{\circ} 43$ 'E], Wong 1663 (K!). INDONESIA. KALIMANTAN SELATAN: Tabalong, upper Tabalong area, PT Aya Yayang Indonesia Concession, km 69, 250 m elev., 7 Aug. 2000, Mogea 7412 (BO, K!, L, NY, WAN); Tabalong, Upper Tabalong Area, PY Aya Yayang Indonesia Concession, Misim, $50 \mathrm{~m}$ elev., [1 ${ }^{\circ} 40^{\prime} \mathrm{S} 115^{\circ} 24^{\prime} \mathrm{E}$ ], 6 Aug. 2000, Mogea 7410 (BO, K!, L, NY, WAN); P.T. Yayang Tanjung, $100 \mathrm{~m}$ elev., 22 Aug. 1985, Anggana 048 (K). KALIMANTAN TIMUR: Bordering Talib's Farm, Lingon, 2 km from Prampus, PT KEM concession, Kelian, upper Mohakan R., 11 

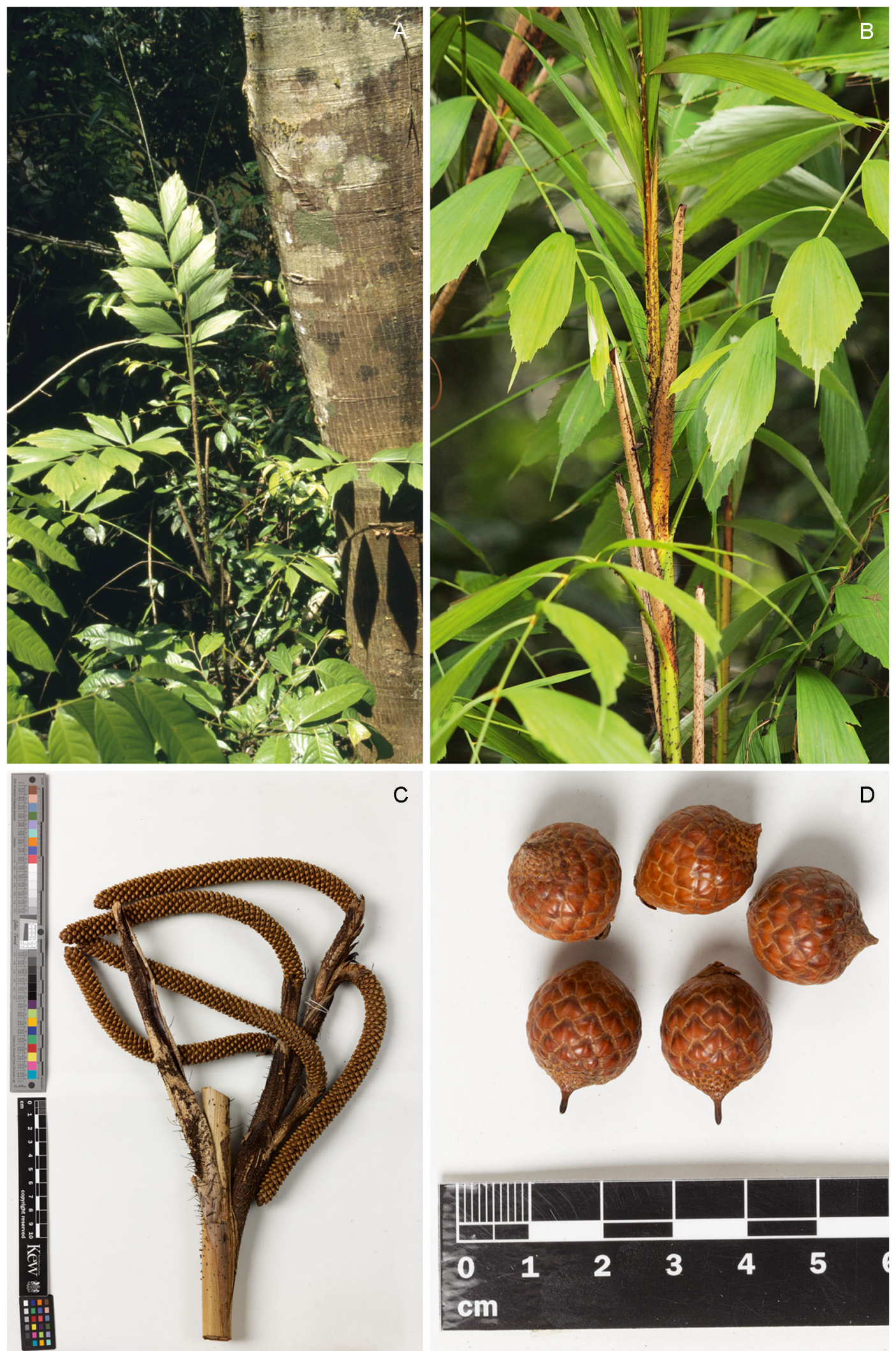

Fig. 11. Korthalsia hispida. A habit; B ocrea; C inflorescence (Dransfield et al. 7494); D fruit (Argent \& Mitchell 9168). 


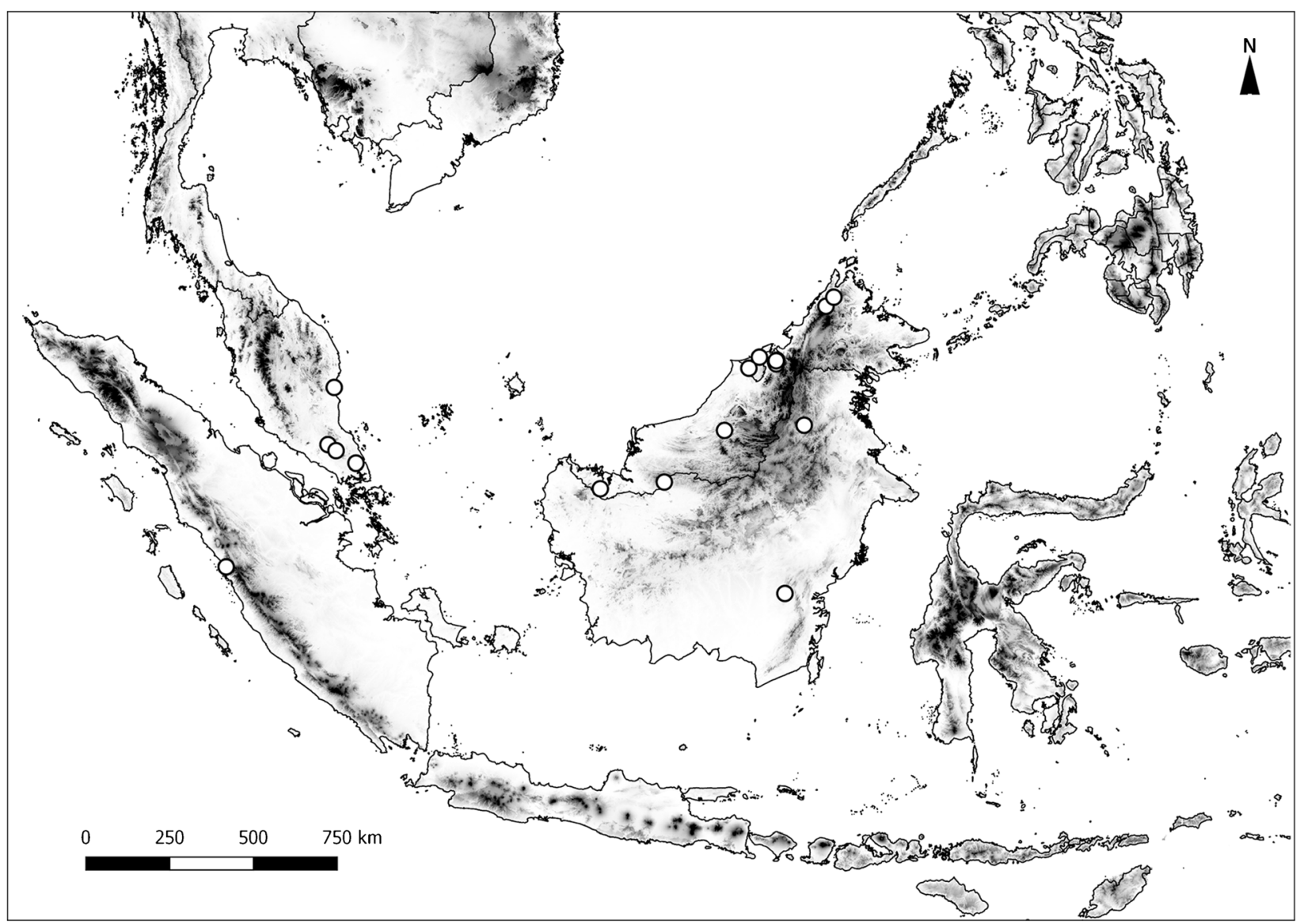

Map 7. Distribution of Korthalsia hispida. Dots are point localities of specimens examined.

Nov. 1991, Stockdale 106 (K!); Surroundings WWF basecamp, Kayan Mentarang Reserve, $400 \mathrm{~m}$ elev., [2 $51^{\circ} \mathrm{N} 115^{\circ} 55^{\prime} \mathrm{E}$ ], 24 Nov. 1991, van Valkenburg 1063 (K!); Berau, RKT 92-93, Inhutani I Concession, $250 \mathrm{~m}$ elev., 8 Dec. 1991, Stockdale 142 (K!). MALAYSIA. SABAH: Telupid, c. 8 miles, Entelebun-Menanam, 150 m elev., 24 Sept. 1979, Dransfield et al. 5803 (K!, SAF, SAR); Tenom, hills above Kampung Baru Jumpa, 700 m elev., 5 Sept. 1979, Dransfield et al. 5598 (K!, KEP!, SAF, SAR); Kampung Kiau Nuluh, [6 $6^{\circ} 2^{\prime} \mathrm{N} 116^{\circ} 29^{\prime} \mathrm{E}$ ], 19 Sept. 1992, Duaneh 118 (K); Kampung Sorinsim, [6 $\left.6^{\circ} 17^{\prime} \mathrm{N} 116^{\circ} 42^{\prime} \mathrm{E}\right], 17$ March 1993, Sibil 162 (K!). SARAWAK: Baram, 4th Division Around Long Selatong Lepo Ga', on hill behind house, banks of Benuon R., 400 m elev., 19 April 1980, Chin 3008 (K!); Belaga, 3rd Division, Sungai Masoh, rumah Nyaving, Kuala Linau, Ulu Belaga, $200 \mathrm{~m}$ elev., [2 $\left.42^{\prime} \mathrm{N} 113^{\circ} 46^{\prime} \mathrm{E}\right], 10$ Aug. 1975, Dransfield et al. 4705 (BH, K!, KEP!, L, SING!); Kapit, upper Rejang R., Clemens 22085 (K!); Kuching, 1st Division, Kampung Kakeng, 200 m elev., [1 $\left.1^{\circ} 9^{\prime} \mathrm{N} 110^{\circ} 27^{\prime} \mathrm{E}\right], 20$ July 1993, Bunker 36 (K!); Tawau, Elphinstone Province, British North Borneo, Oct. 1922, Elmer 21553 (K!); Lubok Antu, 2nd Division,
Delok R., on steep slope in secondary forest near Nanga Sumpa, $200 \mathrm{~m}$ elev., [1 ${ }^{\circ} 20^{\prime} \mathrm{N} 112^{\circ} 10^{\prime} \mathrm{E}$ ], 31 May 1993, Christensen 1188 (K!).

HABITAT. Along steep-sided river valleys in lowland and hill dipterocarp forest at altitudes up to $900 \mathrm{~m}$ above sea level.

VERNACULAR NAMES. Rotan semut (Iban), Paikat ayah pipit (Dayak Lawangan), Wi asas (Iban), Wi semut (Iban), Lesat (Lundaya), Uwai saar (Kenyah), Uei sanam (Kenyah), Laku sepuru (Punan), Ohot (Dayak Tunjung).

USES. Fibre for the plaiting of baskets, craft and binding constructions.

NOTES. Korthalsia hispida and K. robusta are the only two myrmecophilous species in Korthalsia with ocreas that do not clasp the stem, but diverge at an acute angle just above the petiole. These two species are the noisy rattans. The ocreas of $K$. hispida are inhabited by ants of the genus Camponotus (Mattes et al. 1998). The ants make a rhythmic, hissing noise by banging their mandibles against the ocrea when the plant is disturbed. Korthalsia hispida can be distinguished from $K$. robusta by the presence of black spicules on the ocrea and sheaths. 
7. Korthalsia robusta Blume (1843: 170). Type: Indonesia, Sumatra, Praetorius s.n. (holotype L: isotype K!, L!). Korthalsia squarrosa Becc. (Beccari 1909: 620). Type: Philippines, Palawan, Curran 4155 (holotype FI; isotype $\mathrm{K}$ !).

Korthalsia macrocarpa Becc. (Beccari 1918: 149). Type: Indonesia, Borneo, H. Winkler 2777 (holotype FI).

Robust, clustering rattan climbing to $40 \mathrm{~m}$. Stem with sheaths $20-35 \mathrm{~mm}$ diam., without sheaths $14-20 \mathrm{~mm}$ diam.; internodes $24-25 \mathrm{~cm}$. Leaf $1.5-3.0 \mathrm{~m}$ long including cirrus and petiole; sheath bright green to green, with abundant dark brown to brown-black floccose, sparsely armed with scattered black, triangular spines, spines $2-30 \mathrm{~mm}$ long, $1-2 \mathrm{~mm}$ wide; ocrea 16.0 $-41.0 \times 3.0-7.0 \mathrm{~cm}$, conspicuous, diverging from the angle at stem just above the petiole, the margins tending to inroll, papery, brown in colour, armed with abundant 2 - $30 \mathrm{~mm}$ long dark brown spines, black ants usually abundant and very noisy; cirrus $0.50-1.75 \mathrm{~m}$, armed with recurved grapnel spines organised in whorls; petiole $10-35 \mathrm{~cm}, 12-30 \mathrm{~mm}$ wide and $4-6 \mathrm{~mm}$ thick at base, flattened adaxially, abaxially rounded, with slightly grey indumentum, sparsely armed with spines as sheath; rachis $0.65-1.35 \mathrm{~m}$, armed with recurved grapnel spines; 6 - 10 leaflets each side of rachis, regularly arranged, rhomboid to broadly rhomboid, mid-leaf leaflets $20.2-27.8 \times 4.2-13.8 \mathrm{~cm}$, leaflet dull green to dark green above, glabrous, with grey-whitish indumentum under surface, distal margin conspicuously praemorse, transverse veinlets conspicuous, fine and closely spaced c. $0.5 \mathrm{~mm}$. Inflorescence congested, 34.9 $51.0 \mathrm{~cm}$ long including $3.5-15.0 \mathrm{~cm}$ peduncle, branched to 2 orders; prophyll $2.5-8.0 \times 2.2-3.7 \mathrm{~cm}$, with caducous, brown indumentum; rachis bracts up to $12.3-16.2 \times 4.7-8.0 \mathrm{~cm}$, conspicuous, inflated, with caducous, dark brown indumentum and black spicules; primary branches $3,3.0-4.5 \mathrm{~cm}$ apart; proximal primary branch 16.0 - $17.0 \mathrm{~cm}$ long, with up to 2 rachillae; rachillae $13.0-16.0 \mathrm{~cm}$ long and slender, stalk not visible, $1.2-2.0 \mathrm{~cm}$ wide, lacking hairs between rachilla bracts. Flower $8.4-11.0 \times 3.4-5.0 \mathrm{~mm}$ in the bud. Fruit round to oblong, $1.8-2.3 \times 0.9-1.6 \mathrm{~cm}$ covered in $16-$ 18 vertical rows of mid-brown scales with straw-coloured margins. Seed $1.8 \times 1.0 \mathrm{~cm}$; endosperm homogeneous (Figs 1C, 2D, 3D, 12).

DISTRIBUTION. Borneo (Sabah, Sarawak, Central, East and South Kalimantan), Philippines (Palawan), Sumatra (Map 8).

SPECIMENS EXAMINED. SUMATRA. INDONESIA. LAMPUNG: NW of Kota Agung, 350 m elev., [5 ${ }^{\circ} 23$ 'S 104 $25^{\circ} \mathrm{E}$ ], 9 May 1968, Jacobs 8295 (K!); Praetorius s.n. (K!, L!). BORNEO. BRUNeI. TEMBURONG: Batu Apoi National Park, Kuala Belalong Field Centre Jalan Tengah, path to Bukit Belalong, $1 \mathrm{~km}$ from field centre, $300 \mathrm{~m}$ elev.,

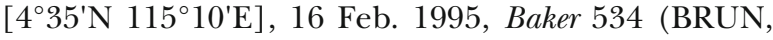
K!, UBD). INDONESIA. KALIMANTAN TENGAH: Bukit Raya, [0 56 'S $112^{\circ} 11$ 'E], Leighton 947 (K!). KALIMANTAN TIMUR: Berau, near km 37 Camp, Inhutani I, $30 \mathrm{~m}$ elev., [2 ${ }^{\circ} 22^{\prime} \mathrm{N} 117^{\circ} 12^{\prime} \mathrm{E}$ ], 18 Aug. 2002, Watanabe 44 (BO, K!); Halfway between Prevab and Mentoko Camps, Kutai Reserve, floodplain of Sangatta R., 20 m elev., 30 Oct. 1991, Stockdale et al. 80 (K); Tabang, Gunung Mendam, KTI, $400 \mathrm{~m}$ elev., [0³6'N $115^{\circ} 54^{\prime} \mathrm{E}$ ], 16 Jan. 1979, Mogea 1629 (K!, KYO); Ma Ancalong, Ma Lun, Sungai Kelinjau, 4 Oct. 1980, Mogea 2708 (K!). KALIMANTAN SELATAN: Sungai Rusi near Mt Sarempaka, 450 m elev., [1 ${ }^{\circ} 49^{\prime} \mathrm{S} 115^{\circ} 44^{\prime} \mathrm{E}$ ], 4 Nov. 1972, de Vogel 2073 (K!); Datar Alai, Pegunungan Meratus Barabai, 350 m elev., 26 Oct. 1972, Dransfield 2967 (BH, K!, L). MALAYSIA. SABAH: Lahad Datu, Masuri, Ulu Segama, [55'N 118¹7'E], 20 Nov. 1949, Cuadra 248 (K!, KEP!); Ranau, Kampung Bundu Tuhan, Kinatongan, [557'N 116 32 E], 7 Sept. 1994, Soibeh 828 (K!); Ranau, Poring, near hot springs, 700 m elev., 29 Aug. 1979, Dransfield et al. 5563 (K!, KEP!, SAP, SAR); Ranau, Kampung Poring, next to Mamut Copper Mine, 3 May 1994, Sambuling 133 (K!); Sandakan, Elopura, Mile 15 state forest, $\left[5^{\circ} 41^{\prime} \mathrm{N}\right.$ $118^{\circ} 6^{\prime} \mathrm{E}$ ], 24 March 1949, Cuadra 2226 (K!, KEP!); Sandakan, Elopura, Sapagaya R., 21 May 1949, Cuadra 2275 (K!, KEP!); Sandakan, Sepilok Forest Reserve, 50 m elev., [5 $52^{\prime} \mathrm{N} 117^{\circ} 55^{\prime} \mathrm{E}$ ], 28 Feb. 1995, Baker et al. 552 (K!); Sandakan, Virgin Jungle Reserve, Mile 42 Segaliud-Lokan Forest Reserve, $50 \mathrm{~m}$ elev., 18 Oct. 1979, Dransfield et al. 5772 (K!, SAF, SAR); Tambunan, Kampung Mansaralong mile 56 1/2, 11 July 1978, Abas SAN 85923 (K!); Kinabatangan Besar, Kori, timber camp, [5 38'N 118 36'E], 2 Nov. 1948, Cuadra 2158 (K!, KEP!); Kota Marudu Distr., Kampung Sorinsim, [6 $\left.{ }^{\circ} 17^{\prime} \mathrm{N} 116^{\circ} 42^{\prime} \mathrm{E}\right], 27$ April 1995, Bakia 339 (K!). SARAWAK: Baram, Sungai Tutoh, $45.72 \mathrm{~m}$ elev., [ $\left.4^{\circ} 0^{\prime} \mathrm{N} 114^{\circ} 48^{\prime} \mathrm{E}\right], 11$ Feb. 1966, Wee-Lek 1086 (K!); Kapit, 7th Division, 8 km Logging Camp, Nanga Mujong, 28 Aug. 1985, Saigol 33 (K!); Miri, Gunung Mulu National Park, 4th Division, on banks of Sungai Melinau near base camp, 30 m elev., 3 Oct. 1977, Dransfield 5262 (K!, NY); Miri, Gunung Mulu National Park, $35 \mathrm{~m}$ elev., [4 ${ }^{\circ}{ }^{\prime} \mathrm{N}$ $114^{\circ} 48^{\prime} \mathrm{E}$ ], 14 Sept. 2015, Shahimi 26 (K!, KEP!); 4th Division, Kelabit Highlands on slope in secondary forest near Ramudu, 900 m elev., [3 ${ }^{\circ} 40^{\prime} \mathrm{N} 115^{\circ} 50^{\prime} \mathrm{E}$ ], 6 Oct. 1993, Christensen 185 (K!). PHILIPPINES. PALAWAN: Balabac Island, [ $7^{\circ} 56^{\prime} \mathrm{N} 117^{\circ} 0$ 'E], 16 Oct. 1906, Merrill 5384 (K!); Kampo 1, 5 March 1984, Madulid 1010 (K); St. Paul's Bay National Park, Lion Cave, 15 m, 7 May 1984, Dransfield 6177 (K!); Pulot III, N of Brooke's Point, $100 \mathrm{~m}$ elev., [855'N 11754'E], 22 May 1984, Dransfield 6206 (K!); Puerto Princesa, Bagumbayan, 22.86 m elev., 18 Nov. 1983, Baja-Lapis 072 (K!); Puerto Princesa, on road between Puerto Princesa 

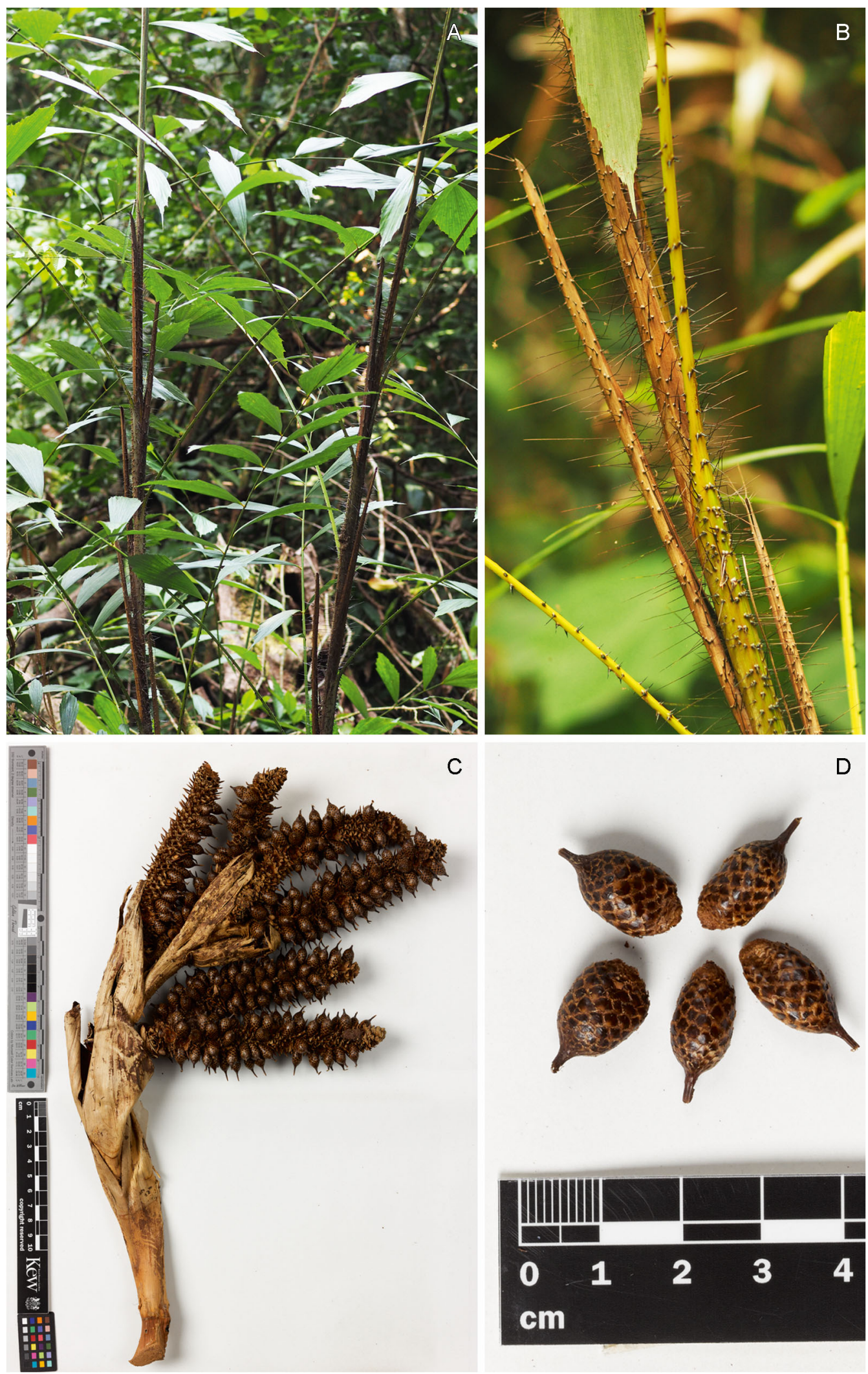

C

D

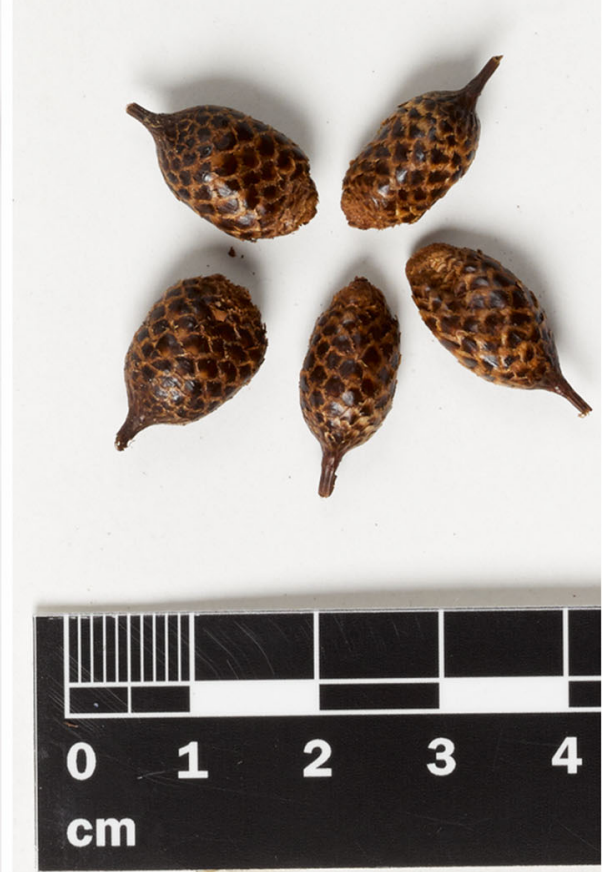

Fig. 12. Korthalsia robusta. A habit; B ocrea; C inflorescence (Dransfield 5262); D fruit (Dransfield 5262). 


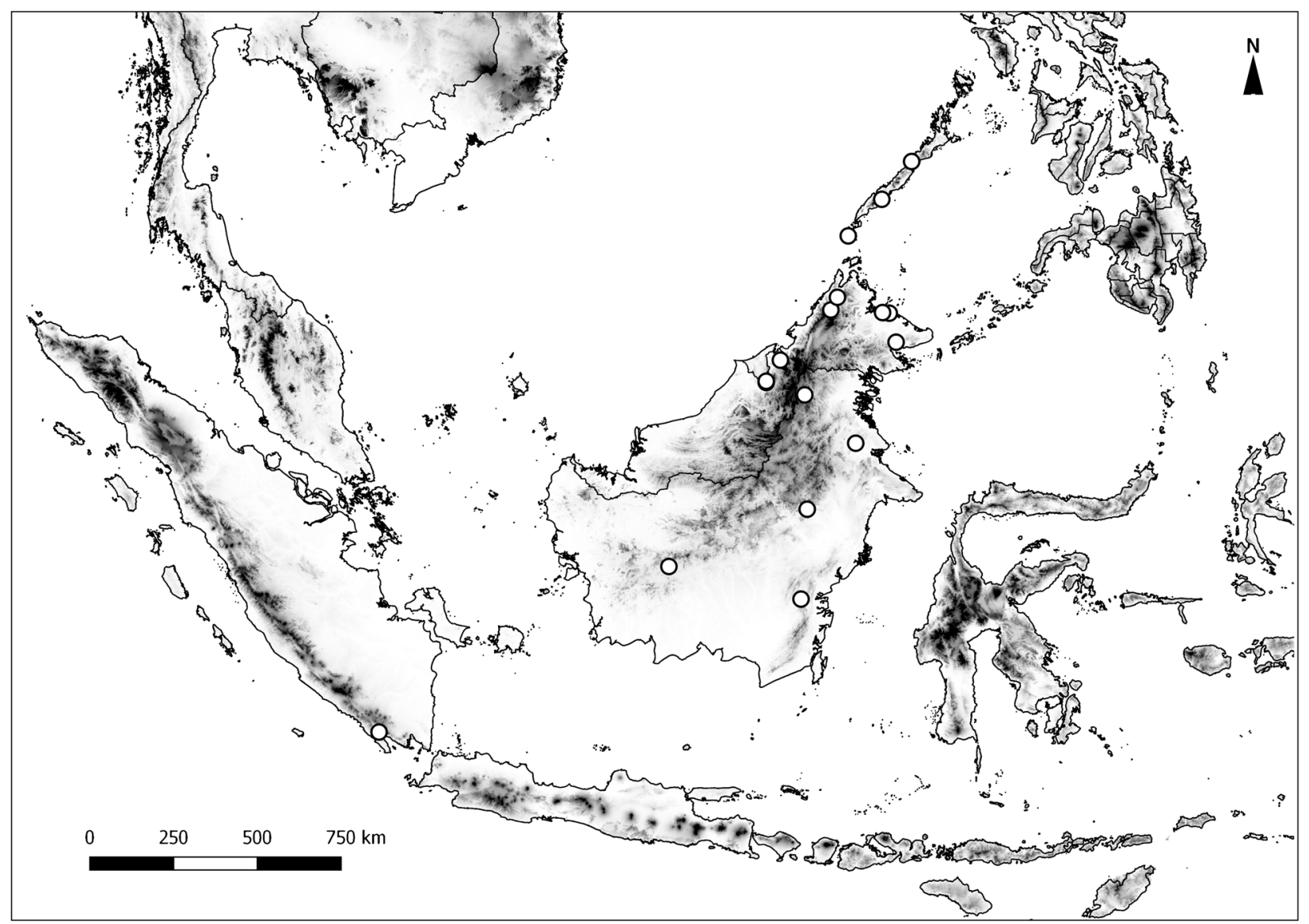

Map 8. Distribution of Korthalsia robusta. Dots are point localities of specimens examined.

and Palawan Consolidated Mining Corporation's Concession, $100 \mathrm{~m}$ elev., [956'N 118 42'E], 11 April 1979, Dransfield 5492 (K!); Curran 4155 (FI, K!).

HABITAT. Lowland dipterocarp forest.

VERNACULAR NAMES. Oros (Kutai dialect), Rotan lasas (Kadazan), Wi semut (Iban), Wae asa (Kelabit language), Wae perah (Penan), Ayas (Pasir language).

USES. Stem used for tying and weaving of baskets, shoots edible, handicraft and construction.

NOTES. Korthalsia robusta is similar to K. hispida but can be easily distinguished from $K$. hispida by the absence of black spicules on the sheaths, ocreas, and bracts, and by the very congested inflorescences. Korthalsia robusta is a noisy rattan and inhabited by ants of the genus Camponotus (Mattes et al. 1998).

8. Korthalsia rostrata Blume (1843: 168). Ceratolobus rostratus (Blume) Becc. (Beccari 1918: 11). Type: Indonesia, Borneo, Sungai Dusun, Muller s.n. (holotype L!). Korthalsia scaphigera Mart. (Martius 1845: 211). Calamosagus scaphiger (Mart.) Griff. (Griffith 1850: 30). Type: Malaysia, Malacca, Griffith s.n. (holotype $\mathrm{BR}$; isotype $\mathrm{K}$ !).
Korthalsia lobbiana H.Wendl. (Wendland 1859: 174). Type: Malaysia, Sarawak, Lobb 106 (holotype K!). Korthalsia machadonis Ridl. (Ridley 1907: 216). Type: Malaysia, Perak, Kamuning. Type not found (Furtado 1951).

Slender, clustering rattan climbing to $20 \mathrm{~m}$. Stem with sheaths $5-15 \mathrm{~mm}$ diam., without sheaths $4-6 \mathrm{~mm}$ diam.; internodes $10-12 \mathrm{~cm}$. Leaf $0.4-1.1 \mathrm{~m}$ long including cirrus and petiole; sheath green, with dark brown to black indumentum, sheath armed with scattered tiny abundant yellow-brown to black triangular spines, spines $1-4 \mathrm{~mm}$ long, 1 - $2 \mathrm{~mm}$ wide; ocrea $25-50 \times 10-30 \mathrm{~mm}$, conspicuous, almost rounded and inflated above the petiole, tough, pale brown to light brown in colour, armed with $2-5 \mathrm{~mm}$ long, tiny triangular brown to dark brown spines, ants abundant within the ocrea; cirrus $0.30-1.25 \mathrm{~m}$, armed with recurved grapnel spines organised in whorls; petiole $2-18 \mathrm{~cm}, 5-19 \mathrm{~mm}$ wide and $2-$ $3 \mathrm{~mm}$ thick at base, flattened adaxially, abaxially rounded, with caducous brown indumentum, sparsely armed with spines as sheath; rachis $0.25-1.45 \mathrm{~m}$, armed with recurved grapnel spines; $4-6$ leaflets each side of rachis, regularly arranged, broadly rhomboid, 

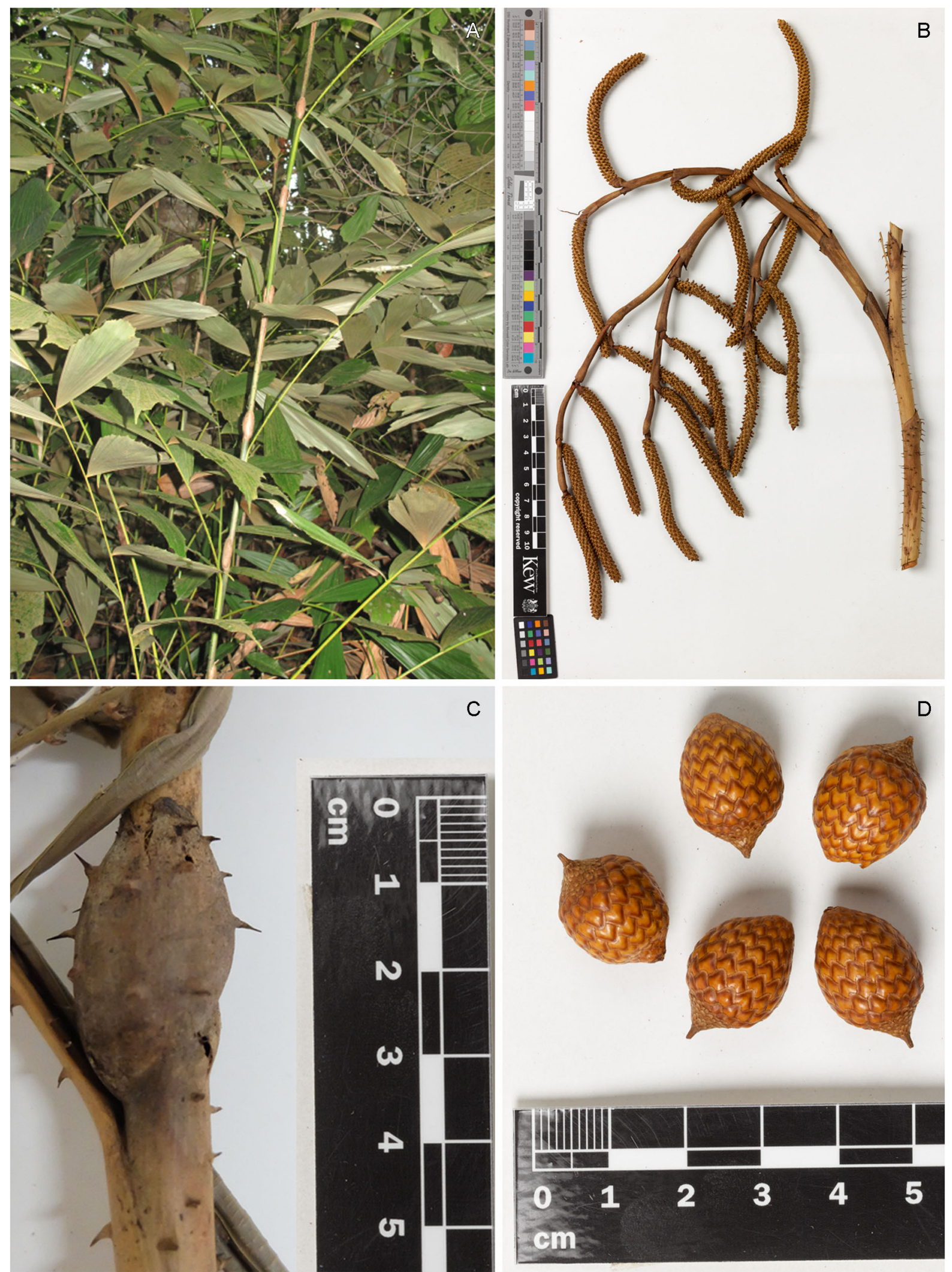

Fig. 13. Korthalsia rostrata. A habit; B inflorescence (Church et al. 1302); C ocrea (Dransfield et al. 6085); D fruit (Laumonier TFB 1155). 
mid-leaf leaflets $13.5-20.5 \times 5.0-6.5 \mathrm{~cm}$, sometimes (rarely) the leaflet narrowly lanceolate, to $21.5-30.8 \times$ $1.5-2.8 \mathrm{~cm}$, leaflet dark green above, glabrous, with caducous, chalky-white indumentum on undersurface, distal margin conspicuously praemorse, transverse veinlets conspicuous, moderately closely spaced, 1.0 $1.5 \mathrm{~mm}$. Inflorescence lax, $35.2-77.5 \mathrm{~cm}$ long including $8.7-9.0 \mathrm{~cm}$ peduncle, branched to 2 orders; prophyll $3.5-6.0 \times 0.7-0.8 \mathrm{~cm}$, with caducous, brown indumentum; rachis bracts up to $4.5-8.7 \times 0.9-3.3$ $\mathrm{cm}$, tightly sheathing, splitting, with caducous, brown indumentum with several tiny spines; primary branches $2-4,4.1-11.2 \mathrm{~cm}$ apart; proximal primary branch $14.7-19.0 \mathrm{~cm}$ long, with up to $2-4$ rachillae; rachillae 12.7 - $18.6 \mathrm{~cm}$ long and slender, including $0.7-1.7 \mathrm{~cm}$ visible stalk, $0.5-0.7 \mathrm{~cm}$ wide, variously hairy between rachilla bracts. Flower $5.0-5.1 \times 2.0-$ $2.2 \mathrm{~mm}$ in the bud. Fruit $2.4-2.5 \times 1.6-1.7 \mathrm{~cm}$ covered in $15-19$ vertical rows of orange-brown scales. Seed $1.1-1.6 \times 0.7-1.0 \mathrm{~cm}$, endosperm ruminate (Figs 1B, 2C, 3C, 13).

DISTRIBution. Malay Peninsula, Borneo (Brunei, Sabah, Sarawak, Central, East and West Kalimantan), Singapore, Sumatra (Map 9).
SPECIMENS EXAMINED. SUMATRA. INDONESIA. ACEH: Aceh Selatan, collected on Gunung Simpang Kiri, 23 Oct. 1997, Sangaji Ẽ Barrow 11 (K!); Aceh Selatan, Kampong Lamee, $72.85 \mathrm{~m}$ elev., [4 ${ }^{\circ} 1^{\prime} \mathrm{N} 96^{\circ} 29^{\prime} \mathrm{E}$ ], 21 Oct. 1997, Sangaji E Barrow 6 (K!). SUMATRA UTARA: E Coast, Silo Maradja, Asahan, May 1927, Bartlett 8132 (K!); E Coast, General region of Marbau, Bilah, near Bilah Pertama (Parbasiran), Feb. 1928, Toroes 200 (K!). SUMATRA BARAT: Sumatra Barat, Sijunjung, Muro Kulampi, 200 m elev., 26 Feb. 1974, Dransfield 3957 (K!); Bengkulu, Ketahun, 40 m elev., 26 Dec. 1984, Uhaedi 019 (K!); Pajakumbuh, Taram, sandstone region of $\mathrm{R}$. Tjampo, $500 \mathrm{~m}$ elev., 23 Aug. 1957, Meijer 6881 (K!). JAMBI: Jambi, Batang Tebopandak, $300 \mathrm{~m}$ elev., [1 ${ }^{\circ} 38^{\prime} \mathrm{S}$ 101 $38^{\circ} \mathrm{E}$ ], Sept. 1993, Trichon 801 (K!). LAMPUNG: Lampung, NW of Kota Agung, $350 \mathrm{~m}$ elev., [5 ${ }^{\circ} 23^{\prime} \mathrm{S} 104^{\circ} 25^{\prime} \mathrm{E}$ ], 18 May 1968, Jacobs 8482 (K!). MALAY PENINSULA. MALAYSIA. JOHOR: Endau, between Bukit Petar and Sungai, 7 Sept. 1987, Gianno 151 (A, BISH, BO, K!, KEP!, L, LAE, P, PNH, SAN, SAR, SING!, TI, TNS, UC, US); Mersing, Gunung Arong Forest Reserve Cpt. 9, 20 m elev., [ $2^{\circ} 32^{\prime} \mathrm{N} 103^{\circ} 47^{\prime} \mathrm{E}$ ] 13 June 1977, Dransfield 5064 (K); Ulu Kahang, 106.68 m elev., 2 June 1923, Holttum 10916 (K!). PAHANG: Aur Forest

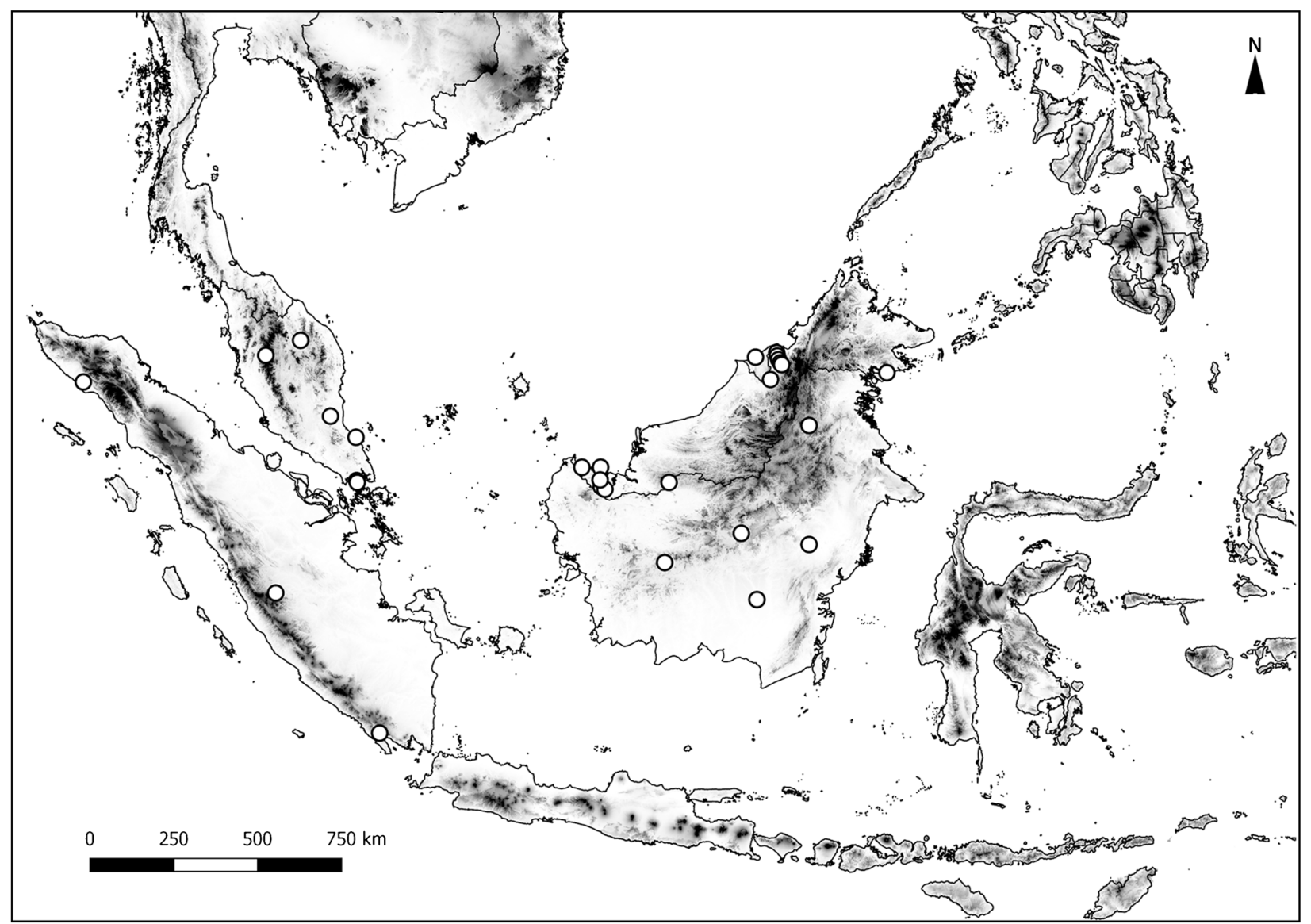

Map 9. Distribution of Korthalsia rostrata. Dots are point localities of specimens examined. 
Reserve, 38 miles inland from Kuala Rompin, $50 \mathrm{~m}$ elev., [3 ${ }^{\circ} 6^{\prime} \mathrm{N} 103^{\circ} 6^{\prime} \mathrm{E}$ ], 24 July 1975, Dransfield 4583 (K!). PERAK: Ipoh, Kinta Hills Forest Reserve, Compt. 78, near Ampang valley bottom, $350 \mathrm{~m}$ elev., 8 July 1975, Dransfield 4477 (K!); Kuala Kangsar, Gunung Bubu Forest Reserve Cpt. 77 Ulu Trong, 250 m elev., [444'N 101 $\left.{ }^{\circ} 22^{\prime} \mathrm{E}\right], 18$ Nov. 1977, Dransfield 5381 (K!); Larut, 152.4 m elev., Oct. 1883, Dr King's collector 5047 (K!); Ridge of main range near Kampung Temakah, downstream from Pos Legap, Sungai Plus, 10 Jan. 1988, Gianno 320 (A, BISH, BO, K!, KEP!, L, LAE, P, PNH, SAN, SAR, SING!, TI, TNS, UC, US). SELANGOR: Semenyih, Sungai Lalang Forest Reserve, $200 \mathrm{~m}$ elev., [3 ${ }^{\circ} 4^{\prime} \mathrm{N} 101^{\circ} 52^{\prime} \mathrm{E}$ ], 22 April 1977, Dransfield 4950 (K!). MELAKA: Griffith s.n. (BR, K!). TEREngGanU: Besut, Ulu Setiu Forest Reserve foothills of Gunung Lawit, $100 \mathrm{~m}$ elev., [5ㅇ'N 102 ${ }^{\circ} 18^{\prime} \mathrm{E}$ ], 3 Aug. 1977, Dransfield 5142 (K!). SINGAPORE. Nee Soon Swamp Forest, [1 ${ }^{\circ} 23^{\prime} \mathrm{N}$ $103^{\circ} 48^{\prime} \mathrm{E}$ ], Rajasegar E Loo 24 (K!); MacRitchie Forest, $17 \mathrm{~m}$ elev., [1 ${ }^{\circ} 20^{\prime} \mathrm{N} 103^{\circ} 49^{\prime} \mathrm{E}$ ], 11 Dec. 2014, Shahimi 25 (K!, SING!). BORNEO. BRUNEI. BELAIT: Sungai Liang, Sungai Liang Arboretum Reserve, [4 $41^{\circ} \mathrm{N} 114^{\circ} 29^{\prime} \mathrm{E}$ ], Wong 137 (K!). TEMBURONG: Temburong R. Valley, in area of river bed near helicopter pad, $50 \mathrm{~m}$ elev., [4 $48^{\circ} \mathrm{N} 115^{\circ} 3 \mathrm{E}$ ], Johns 7282 (K!); Jalan Tengah $1850 \mathrm{~m}$ from $\mathrm{N}$ end, $100 \mathrm{~m}$ elev., [443'N 115²'E], 1 Aug. 1991, Stockdale 14 (K); Amo, Temburong R. upstream from the Wong

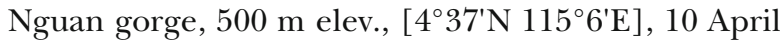
1990, Wong 1738 (K!); Amo, Kuala Belalong, 300 m elev., [4³2'N $115^{\circ} 9^{\prime} \mathrm{E}$ ], 14 Feb. 1992, Dransfield 7064 (K!); Bukit Belalong, N ridge, [ $\left.4^{\circ} 29^{\prime} \mathrm{N} 115^{\circ} 11^{\prime} \mathrm{E}\right], 20$ July 1989, Wong s.n. (K!). INDONESIA. KALIMANTAN TENGAH: near Rekut base camp, $250 \mathrm{~m}$ elev., [0²'S 1146'E], 11 April 1992, Awmack 299 (K!). KALIMANTAN TIMUR: Berau, RKT 92-93, Inhutani I Concession, 250 m elev., 8 Dec. 1991, Stockdale 137 (K!); near Sedulan, c. $50 \mathrm{~km}$ N of Sebulu, 27 Dec. 1978, Mogea et al. 1535 (K!); surrounding WWF basecamp Kayan Mentarang Reserve, $350 \mathrm{~m}$ elev., [2 $51^{\prime} \mathrm{N} 115^{\circ} 55^{\prime} \mathrm{E}$ ], 23 Nov. 1991, van Valkenburg 1055 (K!); about $30 \mathrm{~km}$ N of Sebulu, 30 Dec. 1978, Mogea et al. 1548 (K!); Kalimantan, Sintang, HPH km 86 - 85, along subsidiary road S towards Riam Batang, 80 m elev., [049'S 112³'E], 4 May 1994, Church 1302 (K!); Kelian, K. Equatorial Mining area, $250 \mathrm{~m}$ elev., [0²0'S 115 55'E], 17 March 1997, Kessler 2080 (K!, L). KAlimantan SElatan: Sungai Dusun, [ ${ }^{\circ} 48^{\prime} \mathrm{S}$ $114^{\circ} 31$ 'E], Muller s.n. (L!). MALAYSIA. SABAH: Tawau, St Lucia, Ranggu, Mile 10, State forest, 18 Sept. 1949, Cuadra 2383 (K!, KEP!); Tawau, Membalua Forest Reserve, $50 \mathrm{~m}$ elev., [4ำ $16^{\prime} \mathrm{N} 118^{\circ} 0^{\prime} \mathrm{E}$ ], 3 Nov. 1979, Dransfield et al. 5855 (K!, SAF, SAR); Mile 46, Nabawan, 400 m elev., 11 Sept. 1979, Dransfield et al. 5636 (K!, KEP, L, SAF, SAR); Telupid, c. 5 miles on road Telupid-Karamuak, 24 Oct. 1979, Dransfield et al. 5798 (K!, SAF, SAR). SARAWAK: Lobb 106 (K!); Kapit, 7th Division, $8 \mathrm{~km}$ Logging Camp Nanga Mujong, 28 Aug. 1985, Saigol 35 (K!); Kuching, 1st Division, Sungai Sendok, Proposed Matang National Park, Matang, 10 May 1987, Hock 53881 (K!, L, NY); 1st Division, Wildlife Observation Path, Bako National Park, 19 Aug. 1975, Jawa 36609 (K!, L, NY, SAN); Gunung Gading, Lundu Distr., [1 ${ }^{\circ} 43^{\prime} \mathrm{N} 109^{\circ} 49^{\prime} \mathrm{E}$ ], 17 April 1996, Ara 73906 (K!, SING!); Gunung Santubong, $100 \mathrm{~m}$ elev., [ $\left.1^{\circ} 44^{\prime} \mathrm{N} 110^{\circ} 20^{\prime} \mathrm{E}\right], 6$ June 1964, Ashton 21471 (K!, NY); Lubok Antu, 2nd Division, Delok R., on steep slope in secondary forest near Nanga Sumpa, $150 \mathrm{~m}$ elev., [1 ${ }^{\circ} 20^{\prime} \mathrm{N} 112^{\circ} 10^{\prime} \mathrm{E}$ ], 4 June 1993, Christensen 1183 (K!); Miri, Gunung Mulu National Park, 4th Division, proposed extension, hills near Gunung Buda, $250 \mathrm{~m}$ elev., 14 Oct. 1977, Dransfield 5322 (K!); Miri, Gunung Mulu National Park, 4th Division, lower slopes of Gunung Mulu near Camp 1, $250 \mathrm{~m}$ elev., [4 $\left.5^{\prime} \mathrm{N} 114^{\circ} 533^{\prime} \mathrm{E}\right], 5$ Oct. 1977, Dransfield 5272 (K!, NY, L); Serian, 1st Division, Sabal Tapang Forest Reserve Mile 70, $250 \mathrm{~m}$ elev., 19 May 1981, Dransfield et al. 6085 (K!, L, NY); 4th Division, Dulit Range, 350 m elev., 9 Oct. 1983, Awa 46688 (K!, L); Wooded slopes of Mt Mattang above water works, 31 Dec. 1963, Moore 9084 (K); 5th Division, Path to Bukit Pagon, Limbang, 29 July 1984, Awa 47554 (K!, KEP!); 3rd Division, Sungai Masoh, rumah Nyaving, Kuala Linau, Ulu Belaga, $200 \mathrm{~m}$ elev., 9 Aug. 1975, Dransfield et al. 4680 (BH, K!, KEP, L, SING!); Semenggoh Forest Reserve, 12 miles from Kuching, 20 Dec. 1955, Tomlinson 6 (K); Semenggoh arboretum, [1 ${ }^{\circ} 23^{\prime} \mathrm{N} 110^{\circ} 32^{\prime} \mathrm{E}$ ], 9 Feb. 1995, Baker 514 (K!, KEP!); 1st Division, Kampung Gayu, 250 m elev., [1 ${ }^{\circ} 13^{\prime} \mathrm{N} 110^{\circ} 21$ 'E], 15 July 1993, Bunker 30 (K!); 1st Division, Kampung Kakeng, $200 \mathrm{~m}$ elev., [1 ${ }^{\circ} 9^{\prime} \mathrm{N}$ $110^{\circ} 27^{\prime E}$ ], 20 July 1993, Bunker 33 (K).

HABITAT. Occurs in a variety of habitats including lowland and hill dipterocarp forest and Kerangas forest.

VERNACULAR NAMES. Wi cit (Iban), Wi Semut (Iban), Uwai merah (Dusun), Rotan kawan (Aceh), Wee pak lavo (Kayan), Laku sepuru (Punan), Uei saman (Kenyan), Lempinit ralun (Murut), Wi nang (Iban), Rotan seru (Iban), Rotan semut (Iban).

USES. Stems used for weaving basket and mats.

NOTES. The widespread Korthalsia rostrata is easily confused with the more narrowly distributed $K$. furtadoana, which occurs only in Borneo (Brunei, Kalimantan and Sabah). When fertile, K. rostrata can be distinguished by its rachillae which are slender compared to the robust rachillae of $K$. furtadoana. However, vegetatively the two species are extremely similar, although fine differences can be detected. Korthalsia rostrata tends to have small, almost rounded ocreas compared to $K$. furtadoana. The leaflets of 
$K$. furtadoana also have conspicuous, fine and closely spaced transverse veinlets.

In Brunei, a form of Korthalsia rostrata occurs that has lanceolate rather than rhomboid leaflets. Although the shape of leaflets is distinctive, the form appears otherwise to be identical to remaining forms of $K$. rostrata.

Korthalsia rostrata is inhabited by ants of Camponotus contractus (Moog et al. 2003), and of the genera Dolichoderus and Philidris (Chan et al. 2012).

9. Korthalsia scaphigeroides Becc. (Beccari 1909: 619). Type: Philippines, Mindanao, Zambo-anga, 1 July 1906, Hutchinson 4816 (holotype FI: isotype NY!, US!).

Slender, clustering rattan climbing to c. $5 \mathrm{~m}$. Stem with sheaths c. $10 \mathrm{~mm}$ diam., without sheaths c. $8 \mathrm{~mm}$ diam.; internodes $9-23 \mathrm{~cm}$. Leaf c. $1.5 \mathrm{~m}$ long including cirrus and petiole; sheath brown, with caducous, grey-black indumentum, sheath armed with scattered abundant, brown, triangular spines, spines $2-7 \mathrm{~mm}$ long, c. $2 \mathrm{~mm}$ wide; ocrea $4.5-10.0 \times 1.5-2.5 \mathrm{~cm}$, conspicuous, elongate and inflated, clasping the stem, tough, brown, armed with $1-5 \mathrm{~mm}$ long tiny triangular spines, abundant black ants nested within the ocrea, entry hole made by ants visible; cirrus c. $45 \mathrm{~cm}$, armed with recurved grapnel spines organised in whorls; petiole $9.7-13.0 \mathrm{~cm}, 5-6 \mathrm{~mm}$ wide and c. $3 \mathrm{~mm}$ thick at base, flattened adaxially, abaxially rounded, with brown indumentum as sheath, sparsely armed with spines as sheath; rachis $35-38 \mathrm{~cm}$, armed with recurved grapnel spines; 4 leaflets each side of rachis, regularly arranged, rhomboid, mid-leaf leaflets $17.0-27.0 \times 6.0-7.6 \mathrm{~cm}$, leaflet dark green above, glabrous, with caducous, chalky-white indumentum on undersurface, distal margin conspicuously praemorse, transverse veinlets conspicuous, moderately closely spaced, $1.0-1.5 \mathrm{~mm}$. Inflorescence not seen. Flower not seen. Fruit not seen. Seed not seen (Fig. 14).
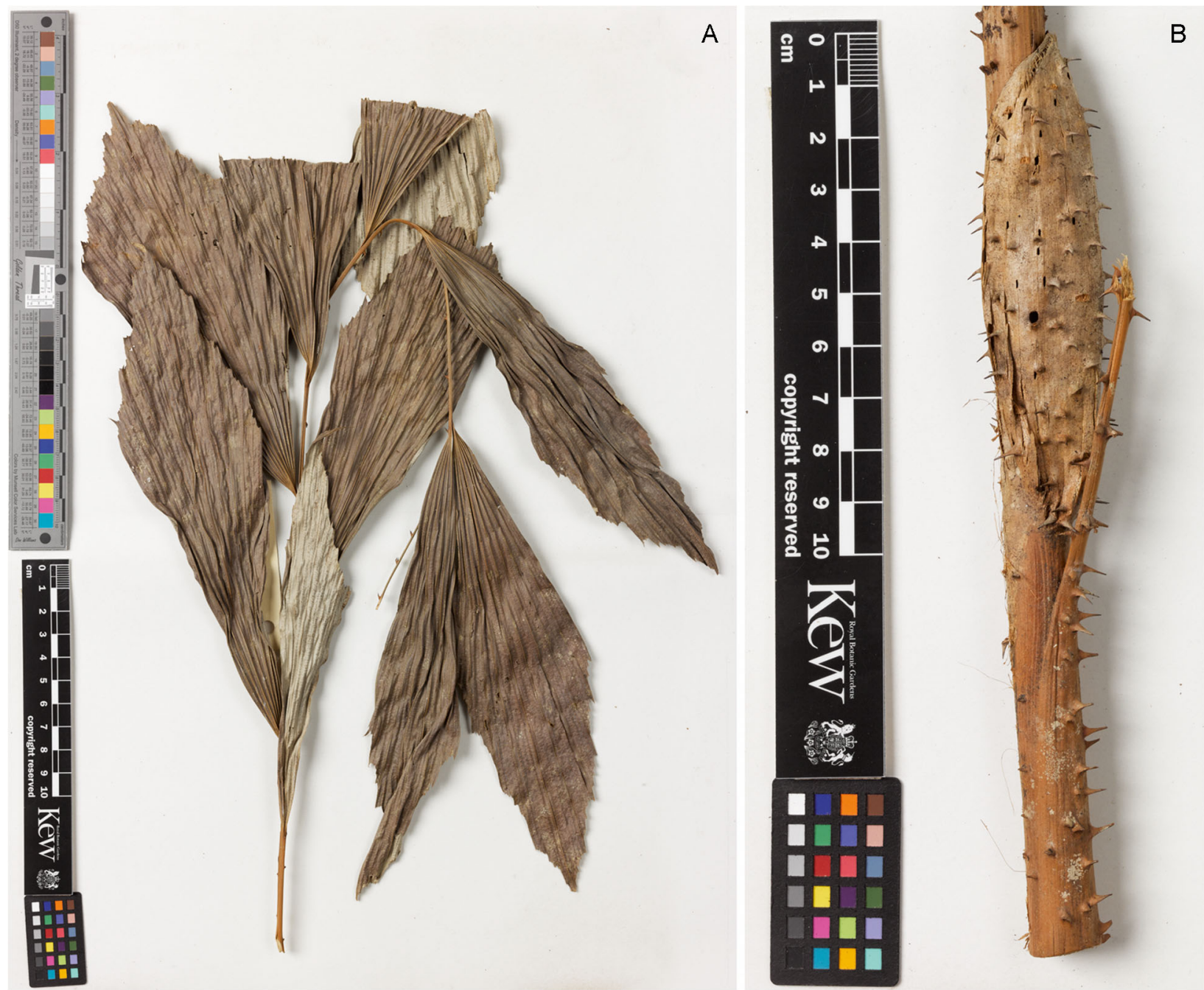

Fig. 14. Korthalsia scaphigeroides. A leaflets (Baja-Lapis 112); B ocrea (Baja-Lapis 112). 
DISTRIBUTION. Philippines (Mindanao) (Map 10). SPECIMENS EXAMINED. PHILIPPINES. MINDANAO: Agusan del Sur, Trento near Simulao, $100 \mathrm{~m}$ elev., [8 ${ }^{\circ} \mathrm{N}$ $126^{\circ} 4^{\prime} \mathrm{E}$ ], 18 July 1984, Fernando 411 (K!); Agusan del Sur, Experimental Forest, FORI, [8 ${ }^{\circ}$ 'N 126 $6^{\circ} \mathrm{E}$ ], BajaLapis 112 (K!); Surigan, April 1915, Ponce 23913 (K!); Zambo-anga, [6 $6^{\circ} 55^{\prime} \mathrm{N} 122^{\circ} 4^{\prime} \mathrm{E}$ ], 1 July 1906, Hutchinson 4816 (FI, NY!, US!).

HABITAT. Growing in dipterocarp forest at low altitude. VERNACULAR NAME. Kaporigid (Philippines).

USES. Furniture and basket making.

NOTES. This species is still known only from sterile material. There is very little herbarium material available. Although Korthalsia scaphigeroides is quite similar to $K$. rostrata, it has ocreas more than $5 \mathrm{~cm}$ longer than those of $K$. rostrata and the abaxial surfaces of leaflets have very dense chalky white indumentum.

10. Korthalsia scortechinii Becc. in J. D. Hooker (1893: 475). Type: Malaysia, Perak, Scortechinis.n. (holotype FI!).

Clustering rattan climbing to $20 \mathrm{~m}$. Stem with sheaths 20 - $25 \mathrm{~mm}$ diam., without sheaths 10 - $15 \mathrm{~mm}$ diam.; internodes $25-30 \mathrm{~cm}$. Leaf $1.0-2.5 \mathrm{~m}$ long including cirrus and petiole; sheath green, almost entirely obscured by ocrea, with caducous, grey-black coloured indumentum, sheath sparsely armed with scattered short brown triangular spines, spines $1-4 \mathrm{~mm}$ long, $1.0-$ $1.5 \mathrm{~mm}$ wide; ocrea $8-15 \times 4-5 \mathrm{~cm}$, conspicuous, swollen and elongate, base of ocrea tightly sheathing, margin tattering (net-like) (for some specimens), clasping the stem, tough, dull pale brown with caducous, chocolate indumentum, armed with $1-3 \mathrm{~mm}$ long, scattered short tiny triangular caducous, chocolate spines, numerous black ants within the ocrea; cirrus $0.30-1.25 \mathrm{~m}$, armed with recurved grapnel spines organised in whorls; petiole $5-10 \mathrm{~cm}, 10-18 \mathrm{~mm}$ wide and c. $4-5 \mathrm{~mm}$ thick at base, flattened adaxially, abaxially rounded, indumentum, sparsely armed with spines; rachis $0.45-1.15 \mathrm{~m}$, armed with recurved grapnel spines organised in whorls; 8 - 13 leaflets each side of rachis, regularly arranged, narrowly rhomboid, mid-leaf leaflets $21.5-30.1 \times 2.5-5.2 \mathrm{~cm}$, leaflet dull to dark green above, glabrous, with whitish indumentum on undersurface, distal margin conspicuously praemorse, transverse veinlets moderately conspicuous, moderately closely spaced, $1.0-1.5 \mathrm{~mm}$. Inflorescence lax, 40.5 - $72.7 \mathrm{~cm}$ long including c. $6 \mathrm{~mm}$ peduncle,

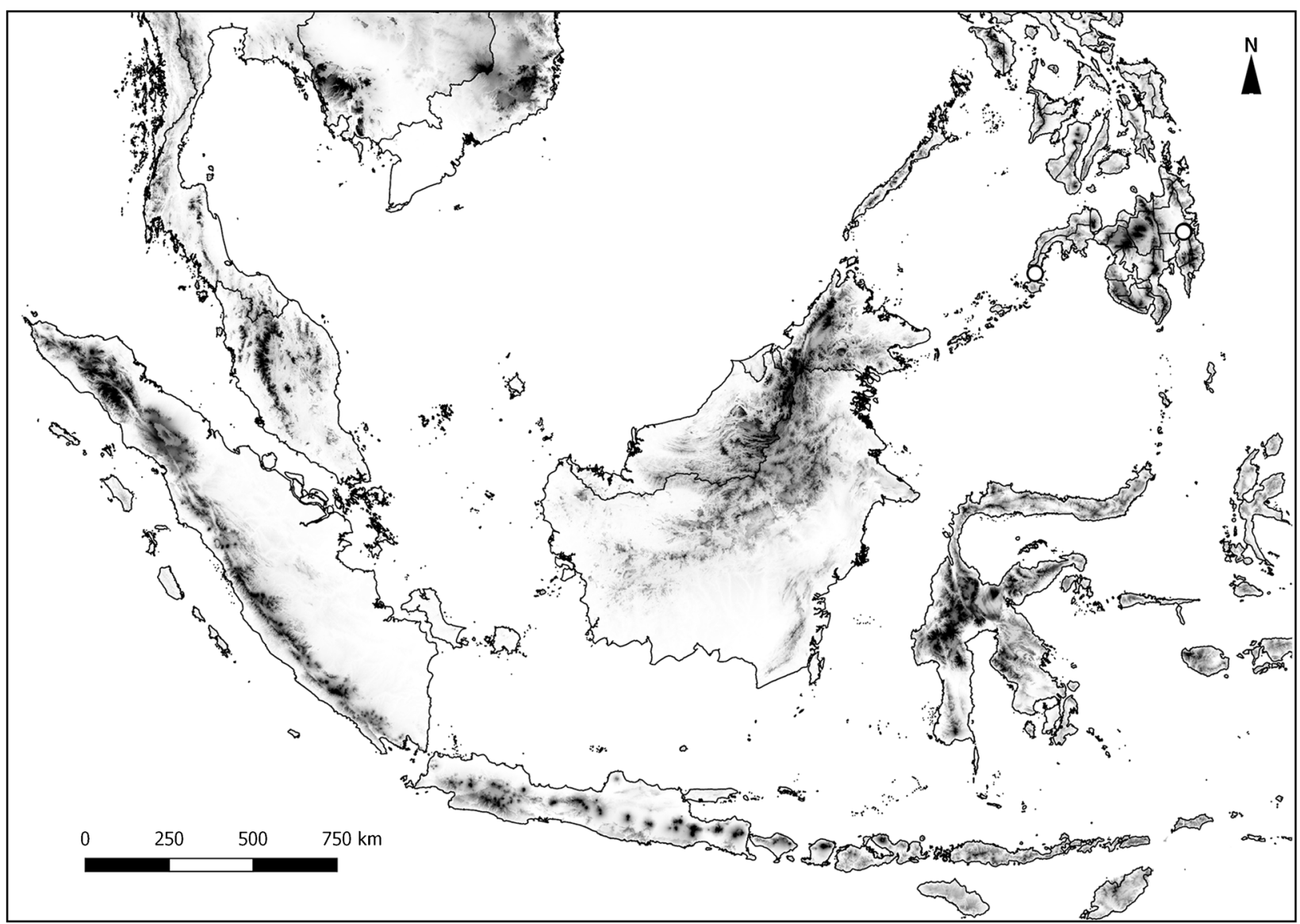

Map 10. Distribution of Korthalsia scaphigeroides. Dots are point localities of specimens examined. 

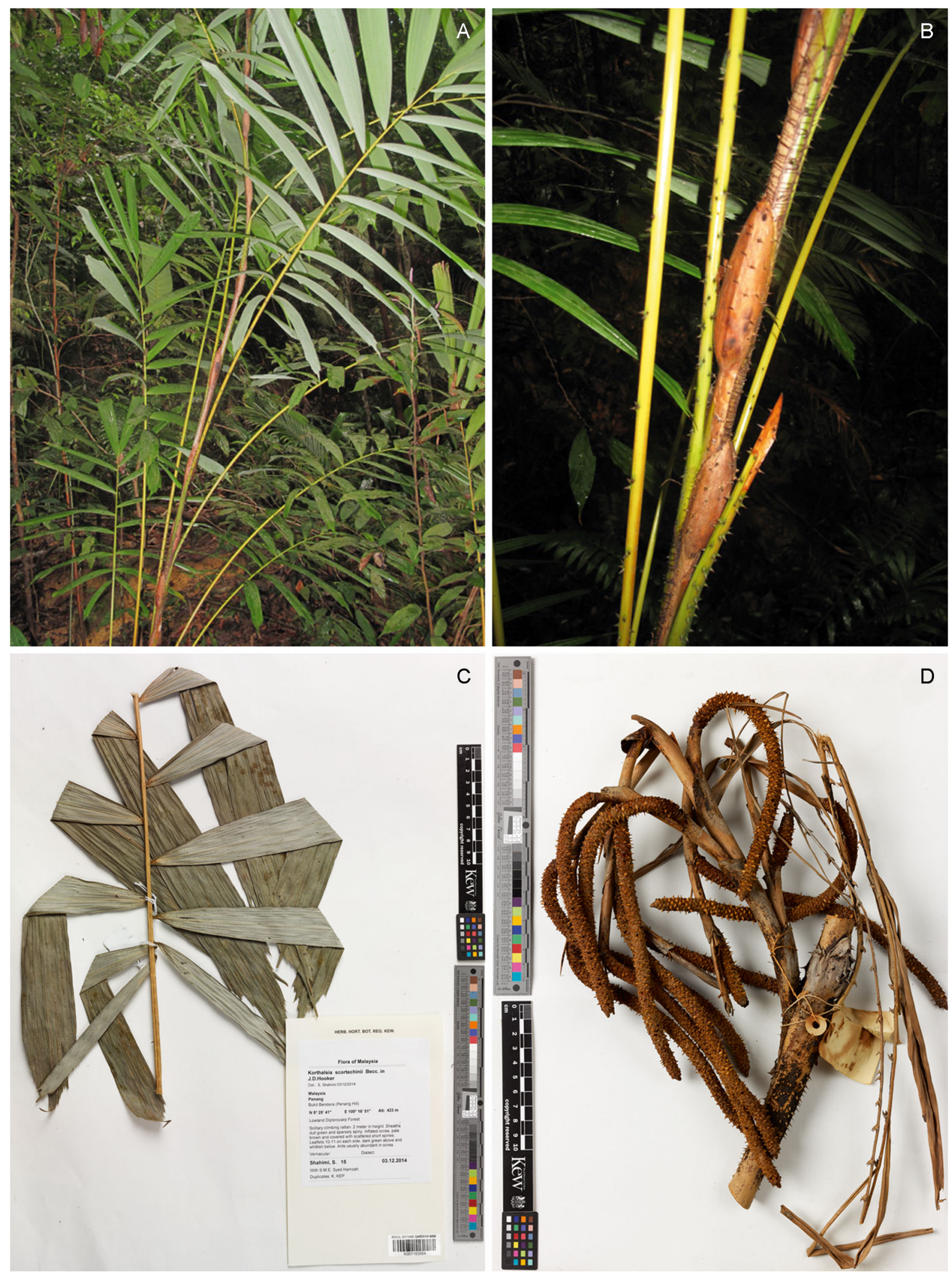

Fig. 15. Korthalsia scortechinii. A habit; B ocrea; C leaflets (Shahimi 15); D inflorescence (Dransfield 4976). 


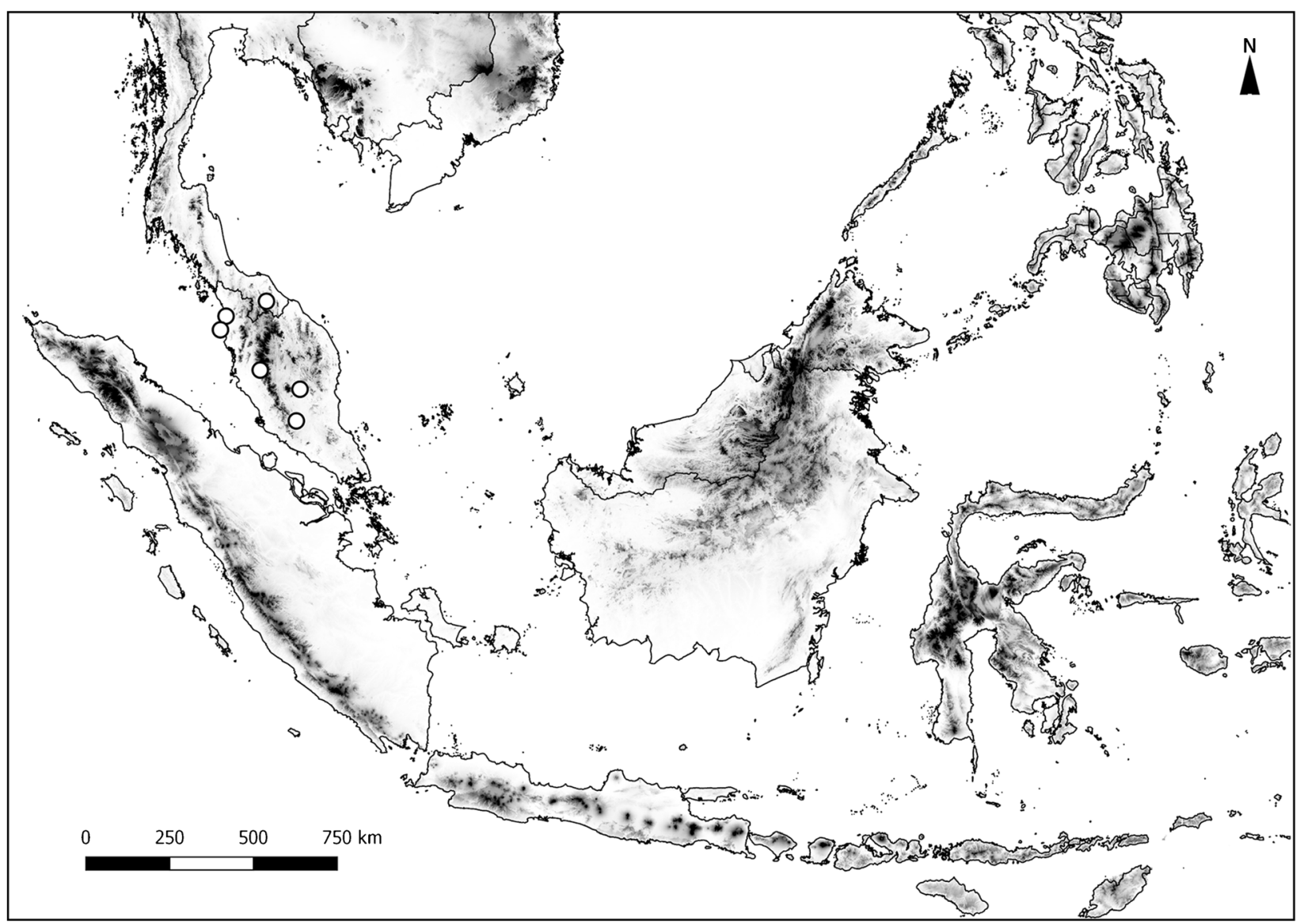

Map 11. Distribution of Korthalsia scortechinii. Dots are point localities of specimens examined.

branched to 2 orders; prophyll c. $4.0 \times 0.7 \mathrm{~cm}$, with caducous, dark brown indumentum; rachis bracts up to $4.1-9.0 \times 1.7-3.7 \mathrm{~cm}$, splitting, with caducous, dark brown indumentum with several tiny spines; primary branches $2-4,5.7-10.6 \mathrm{~cm}$ apart; proximal primary branch $26.2-30.7 \mathrm{~cm}$ long, with up to $2-3$ rachillae; rachillae 14.0 - $28.2 \mathrm{~cm}$ long and slender, including $0.5-$ $1.0 \mathrm{~cm}$ visible stalk, c. $0.7 \mathrm{~cm}$ wide, lacking hairs between rachilla bracts. Flower not seen. Fruit $1.6-1.7 \times 1.1-$ $1.5 \mathrm{~cm}$ covered in $16-19$ vertical rows of orange-brown scales. Seed $1.3 \times 0.7 \mathrm{~cm}$, endosperm ruminate (Figs 2B, 3B, 15).

DISTRIBUTION. Malay Peninsula, Thailand (Map 11). SPECIMENS EXAMINED. THAILAND. NARATHIWAT: Si Sakhon, 550 m elev., [6 ${ }^{\circ} 11^{\prime} \mathrm{N} 101^{\circ} 30^{\prime} \mathrm{E}$ ], 7 March 2001, Niyomdham $\mathcal{E}$ Pudjaa 6446 (K!). MALAY PENINSULA. MALAYSIA. KEDAH: Sungai Patani, Gunung Jerai, $600 \mathrm{~m}$ elev., [5 $47^{\prime} \mathrm{N} 100^{\circ} 25^{\prime} \mathrm{E}$ ], 6 July 1977, Dransfield 5106 (K!). NEGERI SEMBILAN: Pasoh Forest Reserve, $100 \mathrm{~m}$ elev., [2 ${ }^{\circ} 58^{\prime} \mathrm{N} 102^{\circ} 18^{\prime} \mathrm{E}$ ], 9 May 1977, Dransfield 4976 (K!). PAHANG: Genting Highlands, 30 Jan. 1995, Baker 497 (KEP!); Kuala Lipis, 18 Aug. 1925, Somerville 10484 (K!); Tasak Bera, [3⒋ ${ }^{\prime} \mathrm{N} 102^{\circ} 24^{\prime} \mathrm{E}$ ], 3 Sept. 1982, Gianno 512 (K!). PENANG: Penang Hill, 423 m elev.,
[5 $25^{\prime} \mathrm{N} 100^{\circ} 16^{\prime} \mathrm{E}$ ], 3 March 2014, Shahimi 15 (K!, KEP!). PERAK: Tapah, $700 \mathrm{~m}$ elev., [ $4^{\circ} 20^{\prime} \mathrm{N} 101^{\circ} 20^{\prime} \mathrm{E}$ ], 23 Oct. 1982, Ave 178 (K); Scortechini s.n. (FI!).

HABITAT. Lowland and hill dipterocarp forest up to $900 \mathrm{~m}$ altitude.

VERNACULAR NAMES. Rotan dahan (Iban), Rotan semut, Wai doa (Thailand).

USES. Cane used to make baskets, also as binding material.

NOTES. Korthalsia scortechinii has sometimes been confused with $K$. echinometra because of the large swollen inflated ocrea, but can be distinguished by the ocrea bearing numerous short triangular spines (not long narrow spines) and the narrowly rhomboid leaflets (not lanceolate leaflets).

\section{Acknowledgements}

Many individuals and institutes contributed to the completion of this work. We are grateful to the curators and staff of herbaria E, K, KEP and SING for providing access to the specimens. Adrian Loo, Asyraf Mansor, Rahmad Zakaria, Richard Chung and Saw Leng Guan are thanked for their help during fieldwork in Malaysia and Singapore. We are particularly grateful to John 
Dransfield for sharing his encyclopaedic knowledge of rattans. Many other individuals assisted in a wide variety of ways; Abu Husin, Felix Merklinger, Marie-Hélène Weech, Mohamad Aidil, Mohd Nazri, Mohd Sukor, Peter Petoe, Serena Lee, Siti Nur Bazilah, Syed Mohd Ezham, Ummul Nazrah and Wan Nur Fasihah Zarifah. We thank two reviewers and Richard Bateman for helpful comments that have significantly improved the manuscript. This paper is an output of SS's doctoral studies, which were funded by Minister of Higher Education Malaysia and Universiti Malaysia Terengganu.

Open Access This article is distributed under the terms of the Creative Commons Attribution 4.0 International License (http://creativecommons.org/ licenses/by/4.0/), which permits unrestricted use, distribution, and reproduction in any medium, provided you give appropriate credit to the original author(s) and the source, provide a link to the Creative Commons license, and indicate if changes were made.

\section{References}

Baker, W. J., Dransfield, J. \& Hedderson, T. A. (2000). Phylogeny, character evolution, and a new classification of the Calamoid Palms. Syst. Bot. 25: 297 - 322.

\& Dransfield, J. (2002). Calamus longipinna (Arecaceae: Calamoideae) and its relatives in New Guinea. Kew Bull. 57: 853 - 866. \& _ (2006). Field Guide to the Palms of New Guinea. Kew Publishing, Royal Botanic Gardens, Kew.

Beccari, O. (1884). Piante ospitatrici. Malesia, raccolta d'osservazioni lese e papuano 2: 62 - 78.

(1893). Order CLXIII-Palmae. In: J. D. Hooker, Flora of British India 6: 475. L. Reeve, London. (1909). Notes of Philippines palms II. Philipp. J. Sci. 4: $619-620$.

(1918). Asiatic palms - Lepidocaryeae, Part III, The species of Korthalsia. Ann. Roy. Bot. Gard. (Calcutta) 12 (2): $104-155$.

Bequaert, J. C. \& Wheeler, W. M. (1922). Ants in their diverse relations to the plant world. Bull. Amer. Mus. Nat. Hist. 45: 333 - 583.

Blume, C. L. (1843). De Ceratolobo de Korthalsia. Rumphia, sive commentationes botanicae imprimis de plantis Indiae orientalis: tum penitus incognitis tum qua in libris Rheedii, Rumphii, Roxburghii, Wallichii aliorum recensentur 2: 166 - 173.

Chan, C. S-Y., Cheng, J., Loh, J. Y. Q., Tan, E., Loo, A. H. B. \& Tang, J. H. (2012). Observations of ants and aphids in the rattan species Korthalsia echinometra Becc. and Korthalsia rostrata Blume. Raffles Bull. Zool. 25: 133 - 139.

Couvreur, T. L., Kissling, W. D., Condamine, F. L., Svenning, J. C., Rowe, N. P. \& Baker, W. J. (2015).
Global diversification of a tropical plant growth form: environmental correlates and historical contingencies in climbing palms. Front. Genet. 5: 452.

Dransfield, J. (1979). A Manual of the Rattans of the Malay Peninsula. Forest Department, Ministry of Primary Industries, Kuala Lumpur.

(1981). A synopsis of the genus Korthalsia (Palmae: Lepidocaryoideae). Kew Bull. 36: 163 194.

(1984). The Rattans of Sabah. Sabah Forest Record No. 13. Forest Department, Sabah.

(1986). A guide to collecting palms. Ann. Missouri Bot. Gard. 73: 166 - 176.

(1992). The rattans of Sarawak. Royal Botanic Gardens, Kew.

(1997). The rattans of Brunei Darussalam. Ministry of Industry and Primary Resources, Brunei Darussalam.

\& Baker, W. J. (2003). An account of the Papuasian species of Calamus (Arecaceae) with paired fruit. Kew Bull. 58: 371 - 387.

Uhl, N. W., Asmussen, C. B., Baker, W. J., Harley, M. M. \& Lewis, C. E. (2008). Genera Palmarum: The Evolution and Classification of Palms. Kew Publishing, Royal Botanic Gardens, Kew.

Edwards, D. P., Ansell, F. A., Woodcock, P., Fayle, T. M., Chey, V. K. \& Hamer, K. C. (2010). Can the failure to punish promote cheating in mutualism? Oikos 119: 45 - 52.

Furtado, C. X. (1951). Palmae Malesicae, XI —The Malayan species of Korthalsia. Gard. Bull. Singapore 132: $300-324$.

Griffith, W. (1850). Palms of British East India. C. A. Serrao, Calcutta.

Henderson, A. (2009). Palms of southern Asia. Princeton University Press, New York.

\& Nguyen, Q. D. (2013). A new species of Korthalsia (Palmae) from Laos and Vietnam. Palms 57(3): $150-154$.

Holmgren, P. K., Holmgren, N. H. \& Barnett, L. C. (1990). Index Herbariorum. Part I: The herbaria of the world. $8^{\text {th }}$ edition. New York Botanical Garden, New York.

Hooker, J. D. (1893). The flora of British India. Volume 6. L. Reeve \& Co. London.

Lapis, A. C. (2010). A field guide to Philippine rattans. Rushing Water Publisher Ltd., Laguna.

Martius, C. F. P. von. (1845). Historia naturalis palmarum 3 (2): 211. T. O. Weigel, Lipsiae.

Mattes, M., Moog, J., Werner, M., Fiala, B., Nais, J. \& Maschwitz, U. (1998). The rattan palm Korthalsia robusta $\mathrm{Bl}$. and its ant and aphid partners: studies of a myrmecophytic association in the Kinabalu Park. Sabah Parks Nat. J. 1: $47-60$.

Merklinger, F. F., Baker, W. J. \& Rudall, P. J. (2014). Comparative development of the rattan ocrea, a 
structural innovation that facilitates ant-plant mutualism. Pl. Syst. Evol. 300: 1973 - 1983.

Miquel, F. A. W. (1868). De palmis Archipelagi Indici observations novae. Verh. Kon. Akad. Wetensch., Afd. Natuurk. 11 (5): 1 - 33.

Moog, J., Fiala, B., Werner, M., Weissflog, A. \& Maschwitz, U. (2003). Ant-plant diversity in Peninsular Malaysia, with special reference to the Pasoh Forest Reserve. In: T. Okuda, K. Niiyama \& S. Thomas (eds), Pasoh: Ecology and Natural History of a Southeast Asian Tropical Rainforest, pp. 459 - 494. Springer, Tokyo.

Ridley, H. N. (1907). Materials for a Flora of the Malayan Peninsula 2. Methodist Publishing House, Singapore.

Shahimi, S. (2018). Systematics and evolution of the rattan genus Korthalsia Bl. (Arecaceae) with special reference to domatia (PhD thesis). University of Reading, Reading.

Sunderland, T. C. H. (2004). Ant and rattan associations in forest of tropical Africa. Ghana J. Forest. 15(16): $13-19$.
(2012). A taxonomic revision of the rattans of Africa (Arecaceae: Calamoideae). Phytotaxa 51: 1 - 76.

Teijsmann, J. E. \& Binnendijk, S. (1866). Catalogus Plantarum quae in Horto Botanico Bogoriensi Coluntur. Ter Lands-Drukkerij, Batavia.

Vorontsova, M. S., Clark, L. G., Dransfield, J., Govaerts, R. \& Baker, W. J. (2016). World Checklist of Bamboos and Rattans. International Network of Bamboo and Rattan \& Royal Botanic Gardens, Kew.

Wendland, H. (1859). Bot. Zeitung (Berlin) 17: 174 175.

WCSP (2017). World Checklist of Selected Plant Families. Facilitated by the Royal Botanic Gardens, Kew. Published on the Internet; http://apps.kew.org/wcsp/ Retrieved October 27, 2017.

\section{Publisher's Note}

Springer Nature remains neutral with regard to jurisdictional claims in published maps and institutional affiliations. 